\title{
Radionuclide Emission Estimation for the Center for Advanced Energy Studies (CAES)
}

\author{
Bradley Schrader
}

February 2010

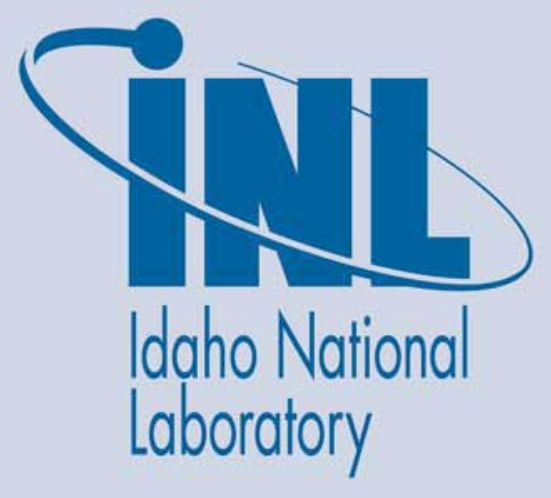

The INL is a U.S. Department of Energy National Laboratory operated by Battelle Energy Alliance 


\section{DISCLAIMER}

This information was prepared as an account of work sponsored by an agency of the U.S. Government. Neither the U.S. Government nor any agency thereof, nor any of their employees, makes any warranty, expressed or implied, or assumes any legal liability or responsibility for the accuracy, completeness, or usefulness, of any information, apparatus, product, or process disclosed, or represents that its use would not infringe privately owned rights. References herein to any specific commercial product, process, or service by trade name, trade mark, manufacturer, or otherwise, does not necessarily constitute or imply its endorsement, recommendation, or favoring by the U.S. Government or any agency thereof. The views and opinions of authors expressed herein do not necessarily state or reflect those of the U.S. Government or any agency thereof. 


\title{
Radionuclide Emission Estimation for the Center for Advanced Energy Studies (CAES)
}

\author{
Bradley Schrader
}

February 2010

Idaho National Laboratory Idaho Falls, Idaho 83415

\author{
http://www.inl.gov
}

Prepared for the

U.S. Department of Energy

Office of Nuclear Energy

Under DOE Idaho Operations Office

Contract DE-AC07-05ID14517 


\section{ABSTRACT}

An Radiological Safety Analysis Computer Program (RSAC)-7 model dose assessment was performed to evaluate maximum Center for Advanced Energy Studies (CAES) boundary effective dose equivalent (EDE, in $\mathrm{mrem} / \mathrm{yr}$ ) for potential individual releases of radionuclides from the facility. The CAES is a public/private partnership between the State of Idaho and its academic research institutions, the federal government through the U.S. Department of Energy (DOE), and the Idaho National Laboratory (INL) managed by the Battelle Energy Alliance (BEA). CAES serves to advance energy security for our nation by expanding educational opportunities at Idaho universities in energy-related areas, creating new capabilities within its member institutions, and delivering technological innovations leading to technology-based economic development for the intermountain region.

CAES has developed a strategic plan (INL/EXT-07-12950) based on the balanced scorecard approach. At the present time it is unknown exactly what processes will be used in the facility in support of this strategic plan. What is known is that the Idaho State University (ISU) Radioactive Materials License (Nuclear Regulatory Commission [NRC] license 11-27380-01) is the basis for handling radioactive material in the facility. The material in this license is shared between the ISU campus and the CAES facility. There currently are no agreements in place to limit the amount of radioactive material at the CAES facility or what is done to the material in the facility.

The scope of this analysis is a summary look at the basis dose for each radionuclide included under the license at a distance of 100,500, and 1,000 m. Inhalation, ingestion and ground surface dose was evaluated using the NRC design basis guidelines. The results can be used to determine a sum of the fractions approach to facility safety. This sum of the fractions allows a facility threshold value (TV) to be established and potential activities to be evaluated against this TV. 


\section{CONTENTS}

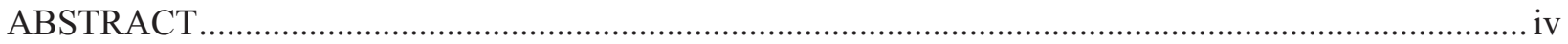

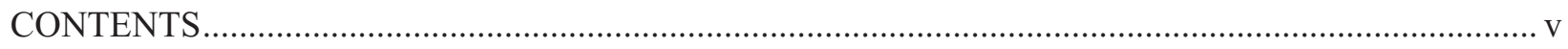

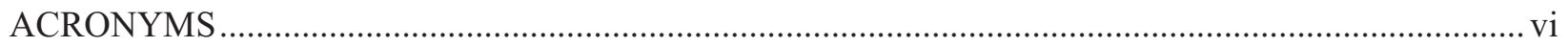

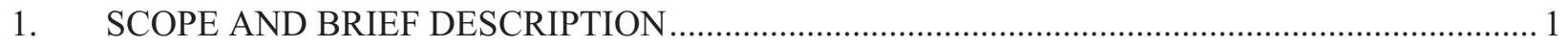

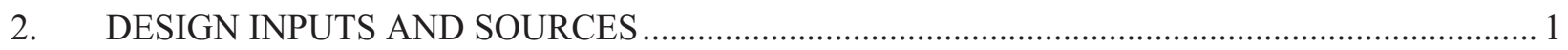

3. RESULTS OF LITERATURE SEARCHES AND OTHER BACKGROUND DATA.................... 1

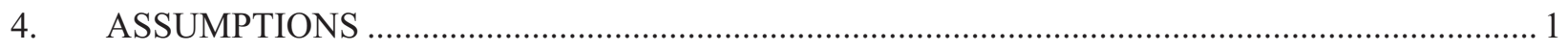

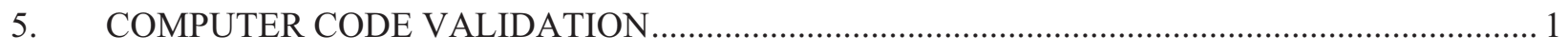

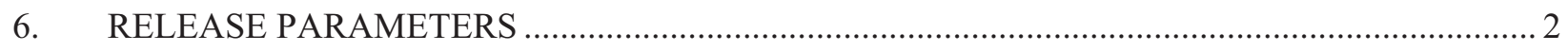

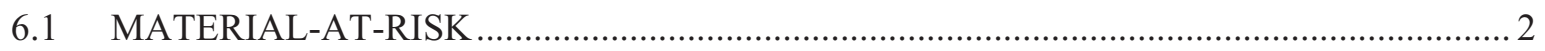

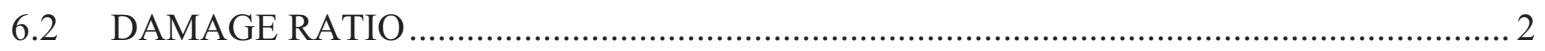

6.3 AIRBORNE RESPIRABLE RELEASE FRACTION ...................................................... 2

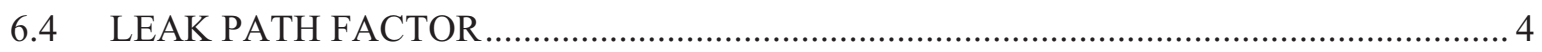

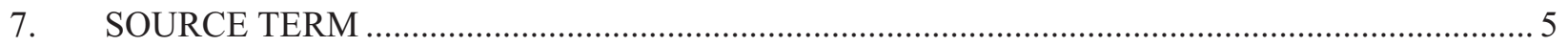

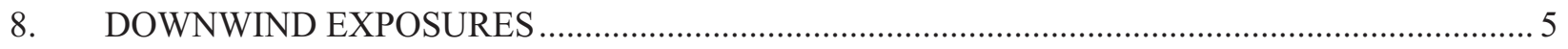

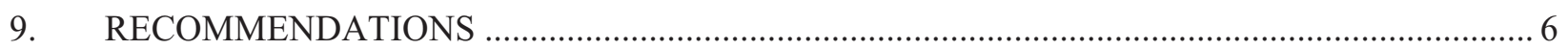

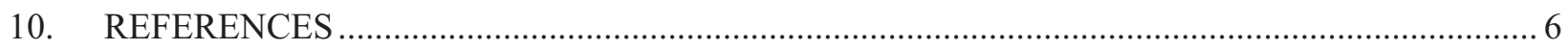

Appendix A RSAC-7 INPUT/OUTPUT AND DOSE RESULTS ……............................................ 7

\section{TABLES}

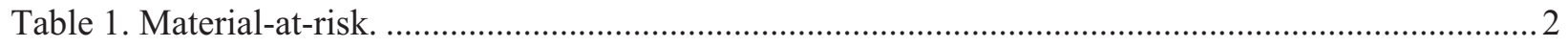

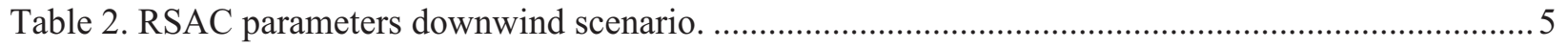

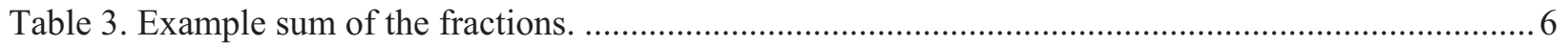




\section{ACRONYMS}

$\begin{array}{ll}\text { ARRF } & \text { airborne respirable release fraction } \\ \text { BEA } & \text { Battelle Energy Alliance } \\ \text { CAES } & \text { Center for Advanced Energy Studies } \\ \text { DOE } & \text { U.S. Department of Energy } \\ \text { DR } & \text { damage ratio } \\ \text { EDE } & \text { effective dose equivalent } \\ \text { INL } & \text { Idaho National Laboratory } \\ \text { ISU } & \text { Idaho State University } \\ \text { LPF } & \text { leak path factor } \\ \text { MAR } & \text { material-at-risk } \\ \text { NRC } & \text { Nuclear Regulator Commission } \\ \text { RSAC } & \text { Radiological Safety Analysis Computer Program } \\ \text { ST } & \text { source term } \\ \text { TV } & \text { threshold value }\end{array}$




\section{Radionuclide Emission Estimation for the Center of Advanced Energy Studies (CAES)}

\section{SCOPE AND BRIEF DESCRIPTION}

An Radiological Safety Analysis Computer Program (RSAC)-7 model dose assessment was performed to evaluate maximum Center for Advanced Energy Studies (CAES) boundary effective dose equivalent (EDE, in mrem/yr) for potential individual releases of radionuclides from the facility. The CAES is a public/private partnership between the State of Idaho and its academic research institutions, the federal government through the U.S. Department of Energy (DOE) and the Idaho National Laboratory (INL) managed by the Battelle Energy Alliance (BEA). CAES serves to advance energy security for our nation by expanding educational opportunities at Idaho universities in energy-related areas, creating new capabilities within its member institutions, and delivering technological innovations leading to technology-based economic development for the intermountain region.

CAES has developed a strategic plan (INL/EXT-07-12950) ${ }^{1}$ based on the balanced scorecard approach. At the present time it is unknown exactly what processes will be used in the facility in support of this strategic plan. What is known is that the Idaho State University (ISU) Radioactive Materials License (Nuclear Regulatory Commission [NRC] license 11-27380-01) ${ }^{2}$ is the basis for handling radioactive material in the facility. The material in this license is shared between the ISU campus and the CAES facility. There currently are no agreements in place to limit the amount of radioactive material at the CAES facility or what is done to the material in the facility.

Therefore, the scope of this analysis is a summary look at the basis dose for each radionuclide included under the license at a distance of 100, 500, and 1,000 m. Inhalation, ingestion and ground surface dose was evaluated using the NRC design basis guidelines ${ }^{3}$ (see Appendix A).

\section{DESIGN INPUTS AND SOURCES}

a. NRC license 11-27380-01, Idaho State University Radioactive Materials License.

b. INL/EXT-07-12950, Center for Advanced Energy Studies (CAES) Strategic Plan

\section{RESULTS OF LITERATURE SEARCHES AND OTHER BACKGROUND DATA}

10 CFR 61 / Appendix D - Method for Estimating Radionuclide Emissions

\section{ASSUMPTIONS}

The release is at ground level. This is due to the low stack height with respect to the facility. NRC mandates that to be considered an elevated release it must be at least 2.5 times the nearest building height. The CAES stack does not meet the criteria of a stack.

Sealed sources (registered pursuant to 10 CFR 32.210 ${ }^{4}$ ) are exempt from this evaluation.

\section{COMPUTER CODE VALIDATION}

a. Dell, Property \#413668

b. Computer program: $\mathrm{RSAC}-7^{45}$

c. Inputs: see Appendix A

d. Outputs: see Appendix A

e. Validation: see http://www.inl.gov/rsac 


\section{RELEASE PARAMETERS \\ 6.1 MATERIAL-AT-RISK}

The material-at-risk (MAR) is the total inventory that could be impacted for a given accident scenario and is expressed in terms of the total quantity at risk. The MAR for the CAES facility could potentially be any of the radionuclides listed in the NRC Radioactive Materials license. Table 1 lists the radionuclides and the maximum quantities allowed under the license.

Table 1. Material-at-risk.

\begin{tabular}{|c|c|}
\hline Byproduct Material and/or Special Nuclear Material & Maximum Amount \\
\hline $\begin{array}{l}\text { Any byproduct material with Atomic Numbers } 3 \text { through } \\
83\end{array}$ & $\begin{array}{l}0.375 \text { curie per radionuclide with a } \\
\text { total possession limit of } 7.5 \text { curies }\end{array}$ \\
\hline $\begin{array}{l}\text { Any byproduct material with Atomic Numbers } 84 \\
\text { through } 103\end{array}$ & $\begin{array}{l}0.007 \text { curie per radionuclide with a } \\
\text { total possession limit of } \\
700 \text { millicuries }\end{array}$ \\
\hline Depleted Uranium & 200 kilograms \\
\hline Natural Uranium & 1 kilogram \\
\hline Uranium - 235 & 5 grams \\
\hline Plutonium - 239 & 0.11 grams \\
\hline Uranium -238 & 7 millicuries \\
\hline
\end{tabular}

\subsection{DAMAGE RATIO}

The damage ratio (DR) represents the fraction of MAR that could be affected by the postulated accident and is a function of the accident initiator and the operational scenario being evaluated. The DR is estimated based upon engineering analysis of the response of structural materials and materials-ofconstruction for containment to the type and level of stress/force generated by the event.

Standard engineering approximations were used. These approximations included a degree of conservatism due to simplification of phenomena to obtain a useable model, but the purpose of the approximation was to obtain, to the degree possible, a realistic understanding of potential effects. The damage ratio for the uncharacterized release scenario is based on conservative estimates. For this evaluation, a conservative damage ratio of 1.0 was selected since at this time no clear defined activities can be analyzed.

\subsection{AIRBORNE RESPIRABLE RELEASE FRACTION}

The airborne respirable release fraction (ARRF) is the fraction of airborne particles that is released during an event which can be transported through air and inhaled into the pulmonary region of the human respiratory system. The particles made airborne under accident induced stresses are dependent upon a variety of factors, such as the bulk density (i.e., how well a powder at rest compacts), the presence of moisture, how effectively the type and level of stress de-agglomerates the powder or subdivides the solid/liquid, the efficiency with which the stress suspends the powder/fragments of solid over varying size ranges, and the degree of immediate proximity of surfaces on which airborne particles may impact/settle. The ARRF includes particles having a 10- $\mu \mathrm{m}$ aerodynamic equivalent diameter or less. The 10 CFR 61 Appendix D reference of, "Environmental Protection Agency (EPA), A Guide for Determining Compliance with the Clean Air Act Standards for Radionuclides Emissions from NRC-Licensed and Non-DOE Federal Facilities," EPA 520/1-89-002, January 1989, was used as the basis for the airborne release fraction. For unknown discrete events, the ARRF was selected as 1.E-3 for particulates and liquids by the following procedure: 


\section{Appendix D to Part 61-Methods for Estimating Radionuclide Emissions}

1. Purpose and Background

Facility owners or operators may estimate radionuclide emissions to the atmosphere for dose calculations instead of measuring emissions. Particulate emissions from mill tailings piles should be estimated using the procedures listed in reference re \#2. All other emissions may be estimated by using the "Procedures" listed below, or using the method described in reference \#1.

\section{Procedure}

To estimate emissions to the atmosphere:

(a) Determine the amount (in curies) used at facilities for the period under consideration. Radioactive materials in sealed packages that remain unopened, and have not leaked during the assessment period should not be included in the calculation.

(b) Multiply the amount used by the following factors which depend on the physical state of the radionuclide. They are:

(i) 1 for gases;

(ii) $10^{-3}$ for liquids or particulate solids; and

(iii) $10^{-6}$ for solids.

If any nuclide is heated to a temperature of 100 degrees Celsius or more, boils at a temperature of 100 degrees Celsius or less, or is intentionally dispersed into the environment, it must be considered to be a gas.

(c) If a control device is installed between the place of use and the point of release, multiply emissions from (b) by an adjustment factor. These are presented in Table 1. 
Table 1-Adjustment to Emission Factors for Effluent Controls

\begin{tabular}{|c|c|c|c|}
\hline Controls & $\begin{array}{c}\text { Types of } \\
\text { radionuclides } \\
\text { controlled }\end{array}$ & $\begin{array}{l}\text { Adjustment } \\
\text { factor to } \\
\text { emissions }\end{array}$ & $\begin{array}{l}\text { Comments and } \\
\text { conditions }\end{array}$ \\
\hline HEPA filters & Particulates & 0.01 & $\begin{array}{l}\text { Not applicable to gaseous } \\
\text { radionuclides; periodic } \\
\text { testing is prudent to ensure } \\
\text { high removal efficiency. }\end{array}$ \\
\hline Fabric filter & Particulates & 0.1 & $\begin{array}{l}\text { Monitoring would be } \\
\text { prudent to guard against } \\
\text { tears in filter. }\end{array}$ \\
\hline Sintered metal & Particulates & 1 & $\begin{array}{l}\text { Insufficient data to make } \\
\text { recommendation. }\end{array}$ \\
\hline $\begin{array}{l}\text { Activated } \\
\text { carbon filters }\end{array}$ & lodine gas & 0.1 & $\begin{array}{l}\text { Efficiency is time } \\
\text { dependent; monitoring is } \\
\text { necessary to ensure } \\
\text { effectiveness. }\end{array}$ \\
\hline \begin{tabular}{|l|} 
Douglas bags: \\
Held one week \\
or longer for \\
decay
\end{tabular} & Xenon & $0.5 / w k$ & $\begin{array}{l}\text { Based on xenon half-life of } \\
5.3 \text { days; }\end{array}$ \\
\hline \begin{tabular}{|l|} 
Douglas bags: \\
Released within \\
one week
\end{tabular} & Xenon & 1 & $\begin{array}{l}\text { Provides no reduction of } \\
\text { exposure to general public. }\end{array}$ \\
\hline $\begin{array}{l}\text { Venturi } \\
\text { scrubbers }\end{array}$ & $\begin{array}{l}\text { Particulates } \\
\text { Gases }\end{array}$ & $\begin{array}{l}0.05 \\
1\end{array}$ & $\begin{array}{l}\text { Although venturis may } \\
\text { remove gases, variability in } \\
\text { gaseous removal efficiency } \\
\text { dictates adjustment factor } \\
\text { for particulates only. }\end{array}$ \\
\hline $\begin{array}{l}\text { Packed bed } \\
\text { scrubbers }\end{array}$ & Gases & 0.1 & $\begin{array}{l}\text { Not applicable to } \\
\text { particulates. }\end{array}$ \\
\hline \begin{tabular}{|l} 
Electrostatic \\
precipitators
\end{tabular} & Particulates & 0.05 & $\begin{array}{l}\text { Not applicable for gaseous } \\
\text { radionuclides }\end{array}$ \\
\hline Xenon traps & Xenon & 0.1 & $\begin{array}{l}\text { Efficiency is time } \\
\text { dependent; monitoring is } \\
\text { necessary to ensure } \\
\text { effectiveness. }\end{array}$ \\
\hline Fume hoods & All & 1 & $\begin{array}{l}\text { Provides no reduction to } \\
\text { general public exposures. }\end{array}$ \\
\hline Vent stacks & All & 1 & $\begin{array}{l}\text { Generally provides no } \\
\text { reduction of exposure to } \\
\text { general public. }\end{array}$ \\
\hline
\end{tabular}

\subsection{LEAK PATH FACTOR}

Leak path factors (LPFs) are assumed to be 1.0 to ensure an unmitigated analysis. The LPF is the fraction of the radionuclides in the aerosol transported through some confinement deposition of filtration mechanism. There can be many LPFs for some accident conditions (e.g., the fraction transported from the package, such as a shipping or storage container, to the cell or enclosure; the fraction leaked from the enclosure or cell to the operating area around the enclosure or room outside the hot cell; the fraction leaked from the room to the building-atmosphere interface). Where multiple leak paths are involved, their cumulative effect is often expressed as one value that is the product of all leak path multiples. The LPF is a calculated or standard value based upon (1) established relationships between size of the particulate material, airborne transport mechanisms, and losses by deposition mechanisms, or (2) specified filtration efficiencies. 
A LPF of 1.0 was selected for the receptor location since the CAES facility is assumed to be breached at ground level. Large particle size and plate out are not accounted for as the plume moves out of the facility, resulting in a very conservative assumption.

\section{SOURCE TERM}

The accident specific parameters used to evaluate the dose to downwind receptors require that certain assumptions be made that modify the dispersion release fraction due to the physical aspects of the release. The five components of the following source-term (ST) equation recommended by the NRC contain the basis for the event analysis. To calculate downwind radiological doses for these scenarios, a ST was determined. The ST is the amount of radioactive material released during the postulated accident scenario. The STs are determined using the following equation:

$$
S T=M A R \times D R \times A R F \times R F \times L P F
$$

where

$\begin{array}{ll}S T & =\text { source term }(\mathrm{Ci}) \\ M A R & =\text { material-at-risk }(\mathrm{Ci}) \\ D R & =\text { damage ratio (no units) } \\ A R F & =\text { airborne release fraction (no units) } \\ R F & =\text { respirable fraction (no units) } \\ L P F & =\text { leak path factor (no units). }\end{array}$

\section{DOWNWIND EXPOSURES}

RSAC-7 was used to quantify the plume dispersion coefficients of the postulated accident. The program is used to calculate the doses of the release of radionuclides to the atmosphere. The meteorological capabilities of RSAC-7 include Gaussian plume diffusion for the Pasquill-Gifford, Hilsmeier-Gifford, and Markee diffusion models. The Markee model is used in this analysis. The RSAC input parameters are summarized in Table 2.

Table 2. RSAC parameters downwind scenario.

\begin{tabular}{ll} 
RSAC Input Parameters & Input Values \\
\hline Release elevation $(\mathrm{m})$ & 0 \\
Stability class & $\mathrm{F}$ \\
Wind speed (m/second) & 1.04 \\
Diffusion coefficient & Markee \\
Downwind receptor distance $(\mathrm{m})$ & $100 \mathrm{~m}, 5.0 \mathrm{~km}$
\end{tabular}

The RSAC-7 program allows the user to specify meteorological conditions at the time of radiological release and to calculate diffusion, dispersion, and depletion factors.

A 95\% meteorology applicable to the CAES facility was used to evaluate the dispersion coefficients. Stability class of $\mathrm{F}$ and a wind speed class of $1.04 \mathrm{~m} / \mathrm{second}$ were used for the RSAC-7 runs. As mentioned previously, a ground release was assumed for this scenario. Values for dry deposition were generated to include plume fallout. Deposition velocities were set to $1.0 \mathrm{E}-3 \mathrm{~m} / \mathrm{s}$ for solids. 
Receptor locations are at $100 \mathrm{~m}$ downwind from the release for determining the offsite public dose. Additional calculations were made at 500 and $1,000 \mathrm{~m}$ for information only. The $100 \mathrm{~m}$ dose should be used.

\section{RECOMMENDATIONS}

The radionuclide by radionuclide contribution to dose at the $100 \mathrm{~m}$ receptor location is listed in Appendix A and should be used as a reference basis for comparison against defined activities. The results of this evaluation can be scaled for comparison against specific activities inside of CAES to determine bounding event consequences. The individual radionuclides may be evaluated for overall facility impact by implementing a sum of the fractions approach to dose assessment.

The sum of the fractions methodology requires establishment of a dose limit or threshold for the facility worker. As an example, the threshold value (TV) will be established as $1 \mathrm{rem}$. The dose as defined in Appendix A for 0.375 Ci of I-129, I-131, Cs-137 are shown in Table 3. Nominal values for evaluation of $.1 \mathrm{Ci}$ of each is defined for evaluation. The dose for a release involving all three radionuclides can be established by a sum of the fractions of dose due to the defined MAR. The example MAR equates to $18 \%$.

Table 3. Example sum of the fractions.

\begin{tabular}{|l|l|l|l|l|}
\hline \multicolumn{1}{|c|}{ Radionuclide } & $\begin{array}{c}\text { Dose from App. A } \\
\text { (rem) }\end{array}$ & $\begin{array}{c}\text { Fraction of MAR } \\
(\mathrm{Ci} / \mathrm{Ci})\end{array}$ & $\begin{array}{c}\text { Fractional Dose } \\
\text { (rem) }\end{array}$ & \multicolumn{1}{|c|}{$\begin{array}{c}\text { Sum of Fraction } \\
\text { (rem) }\end{array}$} \\
\hline I-129 & 0.644 & $0.1 / 0.375=0.267$ & $1.719 \mathrm{E}-1$ & $1.719 \mathrm{E}-1$ \\
\hline $\mathrm{I}-131$ & 0.011 & $0.1 / 0.375=0.267$ & $2.933 \mathrm{E}-3$ & $1.748 \mathrm{E}-1$ \\
\hline Cs-137 & 0.0137 & $0.1 / 0.375=0.267$ & $3.658 \mathrm{E}-3$ & $1.785 \mathrm{E}-1$ \\
\hline & & $\begin{array}{l}\text { Percent of TV } \\
(1 \text { rem })\end{array}$ & $18 \%$ \\
\hline
\end{tabular}

\section{REFERENCES}

1. INL/EXT-07-12950, Center for Advanced Energy Studies Strategic Plan, July 2007.

2. Idaho State University (ISU) Radioactive Materials License (NRC license 11-27380-01).

3. Environmental Protection Agency, "A Guide for Determining Compliance with the Clean Air Act Standards for Radionuclides Emissions from NRC-Licensed and Non-DOE Federal Facilities," EPA 520/1-89-002, January 1989.

4. 10 CFR 32.210, "Registration of Product Information," Code of Federal Regulations, Office of the Federal Register.

5. INL/EXT-09-15275, Radiological Safety Analysis Computer (RSAC)Version 7 User Manual, March 2009. 
Appendix A

\section{RSAC-7 INPUT/OUTPUT AND DOSE RESULTS}




\section{SOURCE TERM :}

Idaho State University U.S. Nuclear Regulatory Commission Radioactive Materials License Number 11-27380-01 as amended (No.16) January 14, 2009.

It was assumed that sealed sources, as registered pursuant to 10 CFR 32.210 were exempt from release modeling.

\section{MODEL INPUT - MET Data (2007 data from NOAA Air Resources Lab, Idaho Falls):}

Stability Class F, Wind Speed $1.04 \mathrm{~m} / \mathrm{s}$.

\section{MODEL INPUT - SOURCE DATA:}

Ground-level point source modeled as single stack with height $=1 \mathrm{~m}$, diameter $=1 \mathrm{~m}$, exit velocity $=0 \mathrm{~m} / \mathrm{s}$

\section{MODEL INPUT - AGRICULTURAL DATA:}

"Rural" food source scenario

Default beef cattle density $(7.19 \mathrm{E}-02 / \mathrm{km})$, milk cattle density $(8.56 \mathrm{E}-03 / \mathrm{km})$, cultivated land fraction (7.15E-02) 
Radiological Safety Analysis Computer Program (RSAC 7.0.3)

Name: INL

Company: Idaho National Laboratory

Serial:

Computer: INL413668 Run Date: 01/26/2010

Run Time: 11:00:09

File: CAES Baseline Analysis.rsac

\section{Input}

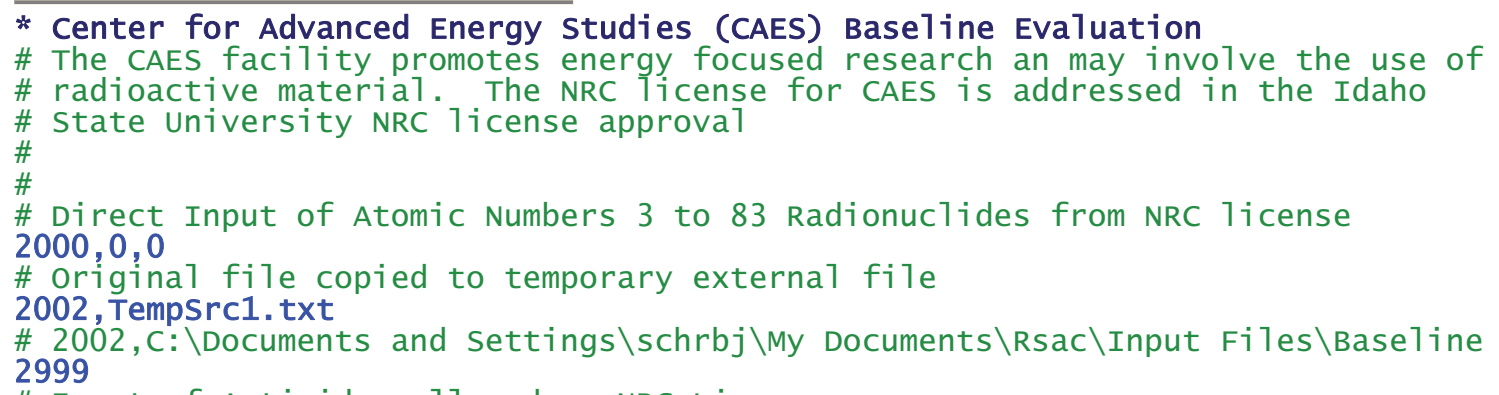

Facility owners or operators may estimate radionuclide emissions to the atmosphere for dose calculations instead of measuring emissions. Particulate emissions from mi11 tailings piles should be estimated using the procedures listed in reference re \#2. A11 other emissions may be estimated by using the "Procedures" listed below, or using $t$ method described in reference \#1.

2. Procedure

To estimate emissions to the atmosphere:

(a) Determine the amount (in curies) used at facilities for the period under consideration. Radioactive materials in sealed packages that remain unopened, and have not leaked during the assessment period should not be included in the calculation.

(b) Multiply the amount used by the following factors which depend on the physical state of the radionuclide. They are:

(i) 1 for gases;

(ii) 10-3for liquids or particulate solids; and

(iii) 10-6for solids. 


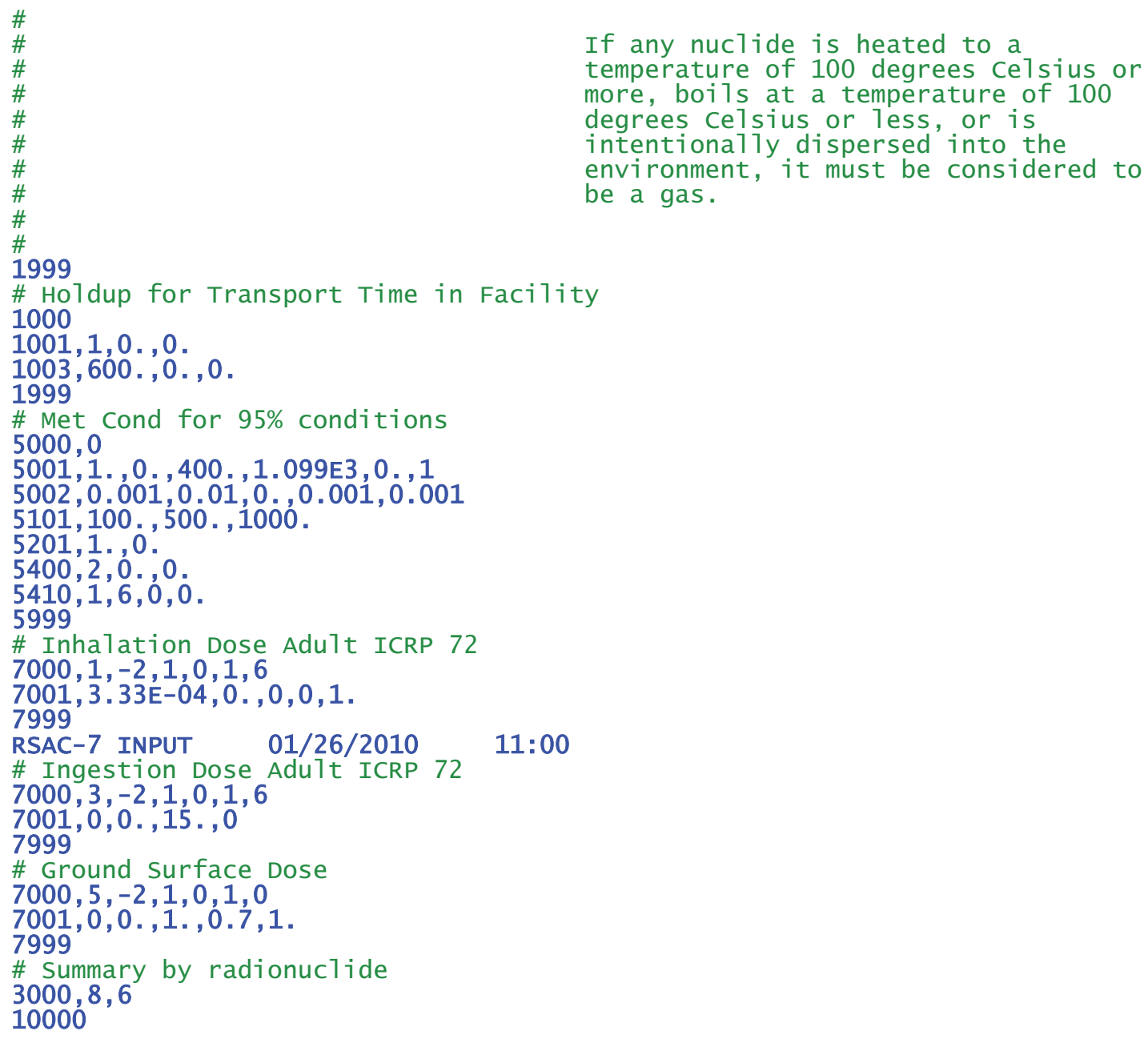




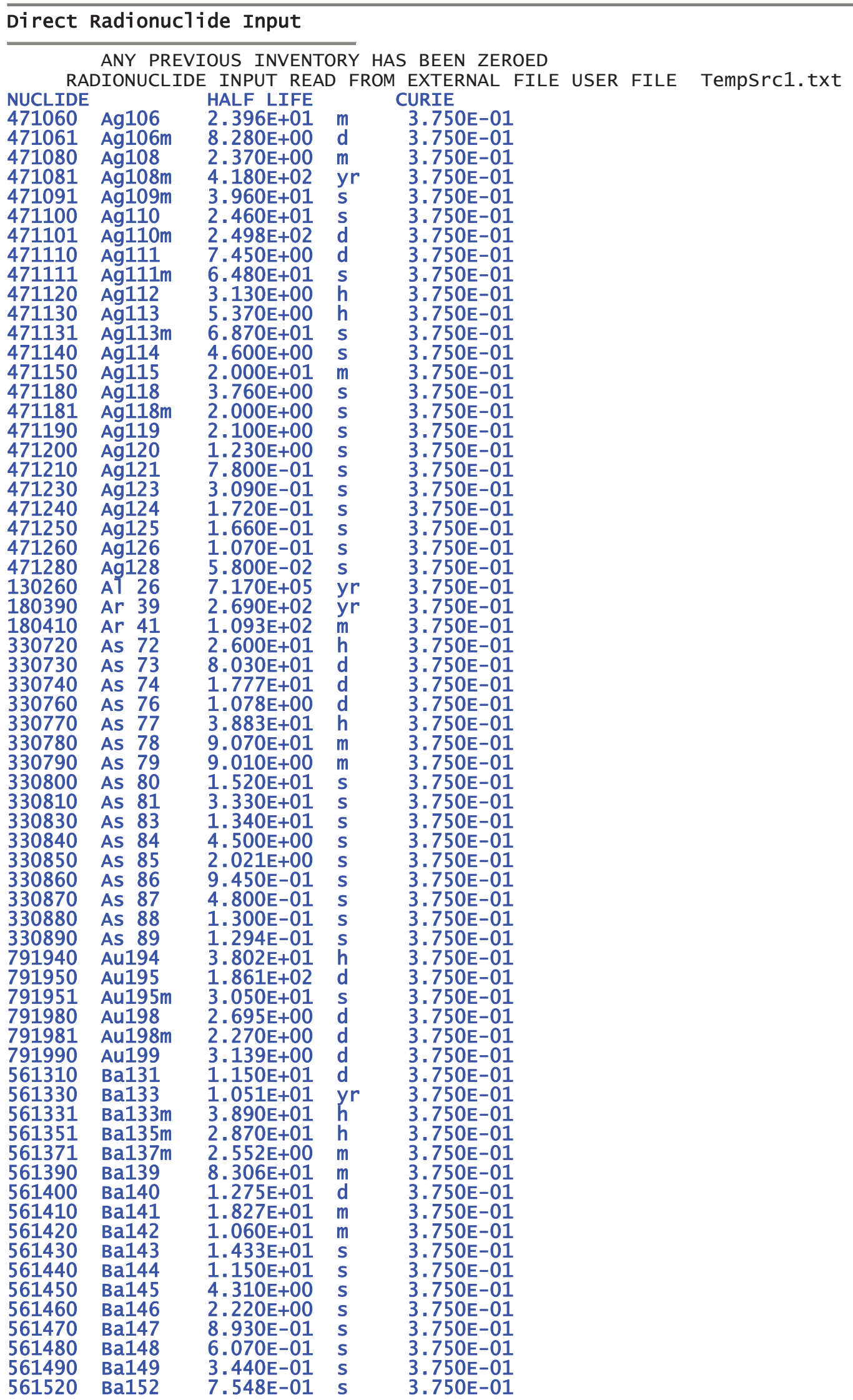




\begin{tabular}{|c|c|c|c|c|}
\hline \multicolumn{2}{|c|}{ NUCLIDE } & \multicolumn{2}{|l|}{ HALF LIFE } & \multirow{2}{*}{$\begin{array}{l}\text { CURIE } \\
3.750 E-01\end{array}$} \\
\hline 40070 & $\mathrm{Be}$ & \multicolumn{2}{|l|}{$5.312 \mathrm{E}+01$} & \\
\hline 40100 & Be 10 & 1. $510 \mathrm{E}+06$ & $y r$ & -01 \\
\hline 832060 & Ві206 & $6.243 \mathrm{E}+00$ & d & $3.750 \mathrm{E}-01$ \\
\hline 832070 & Ві207 & $3.155 \mathrm{E}+01$ & yr & -01 \\
\hline 83208 & Bi208 & $80 E+05$ & $y r$ & $750 \mathrm{E}-01$ \\
\hline 83210 & Ві210 & $5.013 E+00$ & d & $0 \mathrm{E}-01$ \\
\hline 83210 & Bi210m & $3.040 \mathrm{E}+06$ & $y r$ & 3.7 \\
\hline 83211 & Вi211 & $2.140 \mathrm{E}+00$ & m & $0 \mathrm{E}-01$ \\
\hline 832120 & Bi212 & $6.055 \mathrm{E}+01$ & m & OF \\
\hline & Ві213 & $4.559 \mathrm{E}+01$ & m & $0 \mathrm{E}-01$ \\
\hline 40 & Bi214 & $90 \mathrm{E}+01$ & m & \\
\hline & Br 77 & $5.704 \mathrm{E}+01$ & $\mathrm{~h}$ & 3 \\
\hline & Br 80 & $768 \mathrm{E}+01$ & $\mathrm{~m}$ & \\
\hline & $\mathrm{Br} 80 \mathrm{~m}$ & $4.420 E+00$ & h & 3.7 \\
\hline & Br 82 & $30 E+01$ & $\mathrm{~h}$ & \\
\hline & $\mathrm{Br} 82 \mathrm{~m}$ & $30 \mathrm{E}+00$ & m & \\
\hline & $\mathrm{Br} 83$ & $00 \mathrm{E}+00$ & h & 3 \\
\hline & Br 84 & $80 \mathrm{E}$ & m & \\
\hline & $\mathrm{Br} 84 \mathrm{~m}$ & $100 E+00$ & m & \\
\hline & Br 85 & $00 \mathrm{E}+00$ & m & \\
\hline & Br 86 & $.0 E$ & s & \\
\hline & $\mathrm{Br} 86 \mathrm{~m}$ & 4. 500E +00 & $\mathbf{s}$ & 3 \\
\hline & Br 87 & $60 \mathrm{E}+01$ & m & \\
\hline & Br 88 & $34 \mathrm{E}+01$ & $\mathbf{s}$ & U \\
\hline & Br 89 & $4.348 E+00$ & $\mathbf{s}$ & \\
\hline & Br 90 & $\mathrm{E}+00$ & $\mathbf{s}$ & \\
\hline & Br 91 & $10 \mathrm{E}$ & $\mathbf{s}$ & \\
\hline & Br 92 & 3 & s & \\
\hline & Br 93 & 1 & $\mathbf{s}$ & \\
\hline & Br 94 & 2 & $\mathbf{s}$ & \\
\hline & Br 95 & $66 \mathrm{E}$ & S & Uـ \\
\hline & C 11 & & $\mathrm{~m}$ & \\
\hline & C 14 & +03 & $y r$ & -01 \\
\hline 10 & ca 41 & $30 E+05$ & $y r$ & \\
\hline & Ca 45 & 1. & d & \\
\hline & Ca 47 & 4.5 & d & \\
\hline & Ca 49 & $18 \mathrm{E}+00$ & m & \\
\hline & Cd109 & $26 \mathrm{E}+02$ & d & \\
\hline & $\mathrm{cd} 111 \mathrm{~m}$ & $54 \mathrm{E}+01$ & m & \\
\hline & cd113 & 7. & $y r$ & \\
\hline & Cd113m & 1 & yr & \\
\hline & Cd115 & $E+$ & h & \\
\hline & Cd115m & $\mathrm{F}+$ & d & \\
\hline & Cd117 & 2. & $\mathrm{~h}$ & \\
\hline & $\mathrm{cd} 117 \mathrm{~m}$ & $0 \mathrm{E}-$ & h & \\
\hline & $\mathrm{cd} 118$ & 1 & m & \\
\hline & $\mathrm{Cd} 11$ & $E+$ & m & \\
\hline & $\mathrm{cd} 119 \mathrm{~m}$ & 0 & m & \\
\hline & $\mathrm{Cd} 12$ & 5 & $\mathbf{s}$ & \\
\hline & $\mathrm{Cd} 1$ & & S & \\
\hline & $\mathrm{Cd} 1$ & 7 & $\mathbf{s}$ & \\
\hline & $\mathrm{Cd} 1$ & 0 & s & \\
\hline & Cd125 & & $\mathbf{s}$ & \\
\hline & cd126 & & s & \\
\hline & Cd1 & & s & \\
\hline & $\mathrm{Cd} 1$ & & s & \\
\hline & Cd1 & & $\mathbf{s}$ & \\
\hline & Cd1 & & s & \\
\hline & $\mathrm{cd} 1$ & & $\mathbf{s}$ & \\
\hline & $\mathrm{Cd} 1$ & & $\mathrm{~s}$ & \\
\hline & Ce1 & 2 & d & \\
\hline & Ce1 & $50 \mathrm{E}+01$ & d & L \\
\hline & $\mathrm{Ce} 1$ & $\mathrm{E}+$ & yr & \\
\hline & $\mathrm{Ce} 1$ & & h & \\
\hline & $\mathrm{Ce} 1$ & $49 \mathrm{E}+$ & d & ᄃ \\
\hline & $\mathrm{Ce} 1$ & $10 \mathrm{E}+$ & $\mathrm{m}$ & \\
\hline & $\mathrm{Ce}]$ & $2 \angle 5+$ & m & T5- \\
\hline & $\mathrm{Ce}$ & & $s$ & \\
\hline & & & $S$ & \\
\hline & $e$ & & $s$ & \\
\hline
\end{tabular}




\begin{tabular}{|c|c|c|c|c|}
\hline NUCLIDE & & HALF LIFE & & CURIE \\
\hline 58151 & Ce151 & $1.020 \mathrm{E}+00$ & $\mathbf{S}$ & $3.750 \mathrm{E}-01$ \\
\hline 520 & Ce152 & 1. $400 \mathrm{E}+00$ & $\mathbf{S}$ & $50 \mathrm{E}-01$ \\
\hline 530 & Ce153 & $1.725 \mathrm{E}+00$ & S & $50 \mathrm{E}-01$ \\
\hline $5 \varepsilon$ & Ce154 & $3.590 \mathrm{E}-01$ & S & $50 \mathrm{E}-01$ \\
\hline 50 & Ce155 & $7.125 \mathrm{E}-01$ & s & $3.750 \mathrm{E}-01$ \\
\hline 560 & Ce156 & $1.162 \mathrm{E}+00$ & S & $3.750 \mathrm{E}-01$ \\
\hline 570 & Ce157 & $3.618 \mathrm{E}-01$ & s & $0 \mathrm{E}-01$ \\
\hline 60 & C1 36 & $3.010 \mathrm{E}+05$ & yr & $0 \mathrm{E}-01$ \\
\hline 80 & C] 38 & $3.724 \mathrm{E}+01$ & m & $0 \mathrm{E}-01$ \\
\hline 90 & C1 39 & $5.560 \mathrm{E}+01$ & $\mathrm{~m}$ & $0 \mathrm{E}-01$ \\
\hline 60 & Co 56 & 727E+01 & d & 01 \\
\hline 27 & Co 57 & $718 \mathrm{E}+02$ & d & $0 E-01$ \\
\hline 27 & Co 58 & $7.086 \mathrm{E}+01$ & d & $0 E-01$ \\
\hline & Co $58 \mathrm{~m}$ & $040 \mathrm{E}+00$ & h & $3.750 \mathrm{E}-01$ \\
\hline 00 & Co 60 & $5.271 E+00$ & yr & $0 E-01$ \\
\hline 01 & Co $60 \mathrm{~m}$ & $1.047 E+01$ & m & \\
\hline 27 & Co 61 & $1.650 \mathrm{E}+00$ & $\mathrm{~h}$ & 01 \\
\hline & Co $62 \mathrm{~m}$ & 391E+01 & m & 3 \\
\hline 20 & Co 72 & $9.000 \mathrm{E}-02$ & s & $0 \mathrm{E}-0$ \\
\hline 27 & Co 73 & $1.155 \mathrm{E}-01$ & s & \\
\hline 40 & CO 74 & 1 & $\mathbf{s}$ & \\
\hline 27 & Co 75 & $16 \mathrm{E}-02$ & S & 3 \\
\hline & Cr 49 & $30 \mathrm{E}+01$ & $\mathrm{~m}$ & \\
\hline 10 & Cr 51 & $2.770 \mathrm{E}+01$ & d & $U=-U \perp$ \\
\hline 55 & Cs129 & $3.206 \mathrm{E}+01$ & $\mathrm{~h}$ & \\
\hline 55 & Cs131 & $9.689 \mathrm{E}+00$ & d & 01 \\
\hline 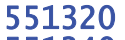 & Cs132 & $6.479 \mathrm{E}+00$ & d & 01 \\
\hline & Cs134 & $2.065 \mathrm{E}+00$ & $y r$ & \\
\hline 55 & Cs134m & $303 E+00$ & $\mathrm{~h}$ & 1 \\
\hline 55 & Cs135 & $00 E+06$ & $\mathrm{yr}$ & \\
\hline & Cs $135 \mathrm{~m}$ & $00 E+01$ & m & 01 \\
\hline ) & Cs136 & $16 \mathrm{E}+01$ & d & \\
\hline 5 & Cs1 & 07E+01 & yr & -00 \\
\hline 380 & Cs13 & $3.341 E+01$ & m & 2 \\
\hline 5 & Cs138m & $10 \mathrm{E}+00$ & m & \\
\hline & Cs1: & $70 \mathrm{E}+00$ & m & 01 \\
\hline ) & Cs1 & $E+01$ & S & \\
\hline ) & Cs1 & 94E+01 & s & \\
\hline 20 & cs1 & $00 \mathrm{E}+00$ & $\mathbf{S}$ & \\
\hline ) & Cs1 & $1.780 \mathrm{E}+00$ & $\mathrm{~s}$ & \\
\hline$)$ & Cs1 & 1. 010E +00 & S & 3 \\
\hline & Cs1 & $40 E-01$ & $S$ & \\
\hline 5 & Cs1 & $10 E-01$ & $\mathrm{~S}$ & \\
\hline & Cs1 & E-01 & $\mathbf{s}$ & \\
\hline ) & Cs148 & $E-01$ & s & \\
\hline & Cu 64 & $E+01$ & h & \\
\hline & Cu 67 & $6.183 \mathrm{E}+01$ & $\mathrm{~h}$ & \\
\hline & Cu 72 & $E+00$ & S & \\
\hline & Cu 73 & 0 & $\mathbf{s}$ & \\
\hline 2 & Cu 74 & 1. & s & \\
\hline 2 & Cu 75 & $\mathrm{E}+00$ & $\mathbf{s}$ & \\
\hline & $\mathrm{Cu} 76$ & $6.410 \mathrm{E}-01$ & s & \\
\hline & Cu 77 & 4 & $\mathbf{S}$ & \\
\hline 2 & Cu 78 & & $\mathbf{S}$ & \\
\hline & Cu 79 & 1 & $s$ & \\
\hline & $\mathrm{Cu} 80$ & 2 & s & \\
\hline & $\mathrm{Cu} 81$ & 47E- & $\mathrm{s}$ & \\
\hline & Dy1 & $E+00$ & $\mathrm{~h}$ & \\
\hline & Dy1 & $44 E+02$ & d & $E-0$ \\
\hline & Dy165 & $4 \mathrm{E}+00$ & $\mathrm{~h}$ & \\
\hline & Dy16 & $60 \mathrm{E}+01$ & h & \\
\hline & Er1 & OOE+00 & d & \\
\hline & $\mathrm{Er}$ & $16 \mathrm{E}+00$ & $\mathrm{~h}$ & L \\
\hline & Eu] & $50 E+01$ & d & 5 \\
\hline & Eu1 & $90 \mathrm{E}+01$ & $y$ & \\
\hline & Eu1 & $80 \mathrm{E}+01$ & h & OF- \\
\hline 6 & Eu1 & $54 E+01$ & $y \mathrm{r}$ & 1 \\
\hline & Eu1 & $12 \mathrm{E}+$ & $h$ & \\
\hline & Eu1 & $\mathrm{E}+00$ & $y r$ & \\
\hline & Eu1 & 61E+00 & $y r$ & \\
\hline
\end{tabular}




\begin{tabular}{|c|c|c|c|c|}
\hline NUCLIDE & & HALF LIFE & & CURIE \\
\hline 63 & Eu156 & 1. 519E+01 & d & $3.750 \mathrm{E}-01$ \\
\hline & Eu157 & $1.518 \mathrm{E}+01$ & $\mathrm{~h}$ & 0 \\
\hline & Eu158 & $4.590 E+01$ & $\mathrm{~m}$ & $0 \mathrm{E}-01$ \\
\hline 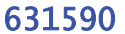 & Eu159 & $1.810 \mathrm{E}+01$ & m & $750 E-01$ \\
\hline 90 & F 18 & $1.098 \mathrm{E}+02$ & $\mathrm{~m}$ & $3.750 \mathrm{E}-01$ \\
\hline 520 & $\mathrm{Fe} 52$ & $8.275 \mathrm{E}+00$ & $\mathrm{~h}$ & $0 E-01$ \\
\hline 50 & $\mathrm{Fe} 5 \overline{5}$ & $2.730 \mathrm{E}+00$ & $y r$ & $0 \mathrm{E}-01$ \\
\hline & $\mathrm{Fe} 59$ & $4.450 \mathrm{E}+01$ & d & $0 \mathrm{E}-01$ \\
\hline 00 & Fe 60 & $1.500 E+06$ & $\mathrm{yr}$ & 01 \\
\hline 60 & Ga 66 & $490 \mathrm{E}+00$ & h & \\
\hline 570 & Ga 67 & $3.261 E+00$ & d & 01 \\
\hline 000 & Ga 68 & $6.763 \mathrm{E}+01$ & m & 01 \\
\hline & Ga 70 & $14 \mathrm{E}+01$ & m & $0 \mathrm{E}-0$ \\
\hline 20 & Ga 72 & $1.410 \mathrm{E}+01$ & $\mathrm{~h}$ & 3 \\
\hline 0 & Ga 73 & $4.860 \mathrm{E}+00$ & $\mathrm{~h}$ & \\
\hline 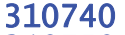 & Ga 74 & $\mathrm{E}+00$ & m & $\mathrm{D}$ \\
\hline & Ga 75 & $1.260 \mathrm{E}+02$ & $\mathbf{s}$ & \\
\hline & Ga 76 & $3.260 \mathrm{E}+01$ & s & \\
\hline 70 & Ga 77 & $20 E+01$ & s & \\
\hline 80 & Ga 78 & 5. & $\mathbf{s}$ & \\
\hline & Ga 79 & $E+00$ & S & 3. \\
\hline & Ga 80 & $1.697 \mathrm{E}+00$ & s & \\
\hline & Ga 81 & $1.217 \mathrm{E}+00$ & s & \\
\hline & Ga 83 & $3.100 \mathrm{E}-01$ & s & \\
\hline & Ga 84 & $8.500 \mathrm{E}-02$ & s & \\
\hline & Gd148 & $7.460 \mathrm{E}+01$ & $\mathrm{yr}$ & 3 \\
\hline & Gd149 & $80 \mathrm{E}+00$ & d & \\
\hline 20 & Gd152 & $1.080 \mathrm{E}+14$ & $y r$ & \\
\hline & Gd153 & $2.404 \mathrm{E}+02$ & d & \\
\hline & Gd159 & $E+01$ & $\mathrm{~h}$ & 3 \\
\hline & Ge 68 & 2.7 & d & 3 \\
\hline 0 & Ge 69 & $05 \mathrm{E}+01$ & $\bar{h}$ & \\
\hline 10 & Ge 71 & 1 & d & \\
\hline 3. & Ge 75 & 8. & m & \\
\hline 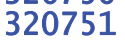 & Ge $75 \mathrm{~m}$ & 4.77 & S & \\
\hline & Ge 77 & $\mathrm{E}+$ & $\bar{h}$ & \\
\hline 71 & Ge $77 \mathrm{~m}$ & $E+01$ & s & \\
\hline 3 & Ge 78 & & m & \\
\hline & Ge 79 & 1. & s & \\
\hline & Ge 80 & 2. & $\mathrm{~s}$ & \\
\hline & Ge 81 & 7. & $\mathbf{s}$ & \\
\hline & Ge 83 & $E+00$ & s & \\
\hline & Ge 84 & 1 & $\mathrm{~s}$ & \\
\hline & Ge 85 & 5. & s & \\
\hline & Ge 86 & -01 & $\mathrm{~s}$ & \\
\hline & Ge 87 & 1. & s & \\
\hline & Ge 88 & & $\mathbf{s}$ & \\
\hline & & 1.233 & $y r$ & 1.5 \\
\hline & Hf17 & 7. & d & $?$ \\
\hline & Hf1 & 5.14 & m & \\
\hline & Hf1 & 2 & ys & \\
\hline & Hf1 & 1 & d & \\
\hline & Hf181 & 4. & d & \\
\hline & Hf182 & $\mathrm{E}+$ & $y r$ & \\
\hline & $\mathrm{Hf} 1$ & $F_{1}$ & h & \\
\hline & $\mathrm{Hg} 1$ & $E+02$ & $y r$ & \\
\hline & & +01 & h & \\
\hline & Hg1 & +01 & $\mathrm{~h}$ & \\
\hline & $\mathrm{Hg} 1$ & 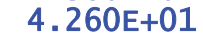 & m & \\
\hline & $\mathrm{Hg} 2$ & 4 & d & \\
\hline & Ho1 & $E+$ & $\mathrm{m}$ & \\
\hline & Hol & $E+01$ & m & \\
\hline & Ho1 & $E+01$ & h & \\
\hline & Ho166m & $E+03$ & $y r$ & \\
\hline 5 & I122 & $\mathrm{E}+$ & $\mathrm{m}$ & \\
\hline & & CIL T & h & \\
\hline & & $E+$ & d & \\
\hline & & & d & \\
\hline & I1 & & d & \\
\hline & I1 & & $m$ & \\
\hline
\end{tabular}




\begin{tabular}{|c|c|c|c|c|}
\hline NUCLIDE & & HALF LIFE & & CURIE \\
\hline 531290 & I129 & $1.570 \mathrm{E}+07$ & yr & $3.750 \mathrm{E}-01$ \\
\hline 00 & I130 & $1.236 \mathrm{E}+01$ & $h$ & $0 \mathrm{E}-01$ \\
\hline 301 & $\mathrm{I} 130 \mathrm{~m}$ & $9.000 \mathrm{E}+00$ & $\mathrm{~m}$ & $3.750 \mathrm{E}-01$ \\
\hline 310 & I131 & $8.021 E+00$ & d & $3.750 \mathrm{E}-01$ \\
\hline 320 & I132 & $2.295 \mathrm{E}+00$ & $\mathrm{~h}$ & $50 \mathrm{E}-01$ \\
\hline 330 & I133 & $80 E+01$ & $\mathrm{~h}$ & 01 \\
\hline & $\mathrm{I} 133 \mathrm{~m}$ & $9.000 \mathrm{E}+00$ & $\mathrm{~s}$ & $0 \mathrm{E}-0$ \\
\hline 40 & I 134 & $5.250 \mathrm{E}+01$ & m & $3.750 \mathrm{E}-01$ \\
\hline 341 & $\mathrm{I} 134 \mathrm{~m}$ & $3.600 \mathrm{E}+00$ & $\mathrm{~m}$ & $750 \mathrm{E}-01$ \\
\hline 50 & I135 & $6.570 \mathrm{E}+00$ & h & 01 \\
\hline 60 & I136 & $10 \mathrm{E}+01$ & $\mathrm{~s}$ & 01 \\
\hline & $\mathrm{I} 136 \mathrm{~m}$ & $90 \mathrm{E}+01$ & $\mathrm{~s}$ & 7 \\
\hline 70 & I137 & $2.450 \mathrm{E}+01$ & S & $750 \mathrm{E}-01$ \\
\hline 80 & I138 & $6.490 \mathrm{E}+00$ & $\mathrm{~s}$ & 01 \\
\hline 390 & I139 & $90 \mathrm{E}+00$ & $\mathbf{s}$ & U \\
\hline 400 & I140 & $0 E-01$ & $\mathrm{~s}$ & \\
\hline 10 & I141 & $00 \mathrm{E}-01$ & s & $50 \mathrm{E}-01$ \\
\hline 420 & I142 & $1.960 \mathrm{E}-01$ & s & $750 E-01$ \\
\hline 0 & I143 & $80 \bar{E}-01$ & $\mathrm{~s}$ & \\
\hline 440 & I144 & E-01 & s & U \\
\hline 110 & In111 & $\bar{E}+00$ & d & \\
\hline 131 & In $113 \mathrm{~m}$ & $1.658 \mathrm{E}+00$ & $\mathrm{~h}$ & $0 \mathrm{E}-01$ \\
\hline 140 & In114 & $7.190 \mathrm{E}+01$ & $\mathrm{~s}$ & \\
\hline 141 & In $114 m$ & $4.951 E+01$ & d & 01 \\
\hline 150 & In115 & $\mathrm{L} \mathrm{E}+14$ & $y r$ & \\
\hline 151 & In $115 \mathrm{~m}$ & $86 \mathrm{E}+00$ & $h$ & 3 \\
\hline 1161 & In116m & $29 E+01$ & m & 01 \\
\hline 170 & In117 & $0 \mathrm{E}+01$ & $\mathrm{~h}$ & \\
\hline L71 & In $117 \mathrm{~m}$ & $2 E+02$ & $\mathrm{~h}$ & 3 \\
\hline 180 & In118 & $0 \mathrm{E}+00$ & S & \\
\hline 1181 & In $118 \mathrm{~m}$ & $50 \mathrm{E}+00$ & m & 3 \\
\hline 491191 & In119m & $00 \mathrm{E}+01$ & m & \\
\hline 00 & In120 & $\mathrm{E}+00$ & S & \\
\hline 1201 & In $120 \mathrm{~m}$ & $E+01$ & $\mathrm{~s}$ & \\
\hline 10 & In121 & $E+01$ & $\mathrm{~s}$ & \\
\hline 491211 & In $121 \mathrm{~m}$ & $80 E+00$ & m & UI \\
\hline 491230 & In 123 & $80 \mathrm{E}+00$ & s & \\
\hline 31 & In $123 \mathrm{~m}$ & 4. & $\mathrm{~s}$ & \\
\hline 491240 & In124 & $\mathrm{E}+00$ & S & \\
\hline 491250 & In125 & $\mathrm{E}+00$ & $\mathrm{~s}$ & \\
\hline 491251 & In $125 \mathrm{~m}$ & $20 \mathrm{E}+01$ & s & \\
\hline 491260 & In126 & $E+00$ & $\mathrm{~s}$ & \\
\hline 270 & In127 & 1 . & $\mathrm{s}$ & \\
\hline 491271 & In $127 \mathrm{~m}$ & $E+00$ & S & \\
\hline 491280 & In128 & $E-01$ & s & \\
\hline 491290 & In12 & $\mathrm{E}-01$ & $\mathbf{s}$ & \\
\hline 491300 & In130 & & s & \\
\hline 1210 & In131 & 2. & s & \\
\hline 1320 & In132 & 2. & $\mathbf{s}$ & \\
\hline 491330 & In133 & E- & s & \\
\hline 491340 & In134 & & s & \\
\hline 771900 & Ir190 & 1. & d & \\
\hline 771901 & Ir $190 \mathrm{~m}$ & 1 . & $\mathrm{h}$ & \\
\hline 771904 & Ir190n & $E+00$ & $\mathrm{~h}$ & \\
\hline 771920 & Ir192 & $E+01$ & d & \\
\hline & Ir $192 m$ & $E+02$ & $y r$ & \\
\hline 771940 & Ir194 & & h & \\
\hline 1041 & Ir $194 m$ & 1 . & d & \\
\hline 190400 & K 40 & 1. & $y$ & \\
\hline 190420 & K 42 & & h & \\
\hline 190430 & K 43 & 2 & $\mathrm{~h}$ & ᄂ \\
\hline & Kr 79 & $4 \mathrm{E}+01$ & $\mathrm{~h}$ & \\
\hline 36081 & $\mathrm{Kr} 81$ & & y & \\
\hline 31 & $\mathrm{Kr} 83 \mathrm{r}$ & 1 . & h & 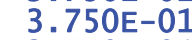 \\
\hline 36 & $\mathrm{Kr} 8$ & 1. & $y r$ & \\
\hline & $\mathrm{Kr} 8$ & $0 \mathrm{E}+00$ & h & 7 \\
\hline & $\mathrm{Kr}$ & & m & \\
\hline & $\mathrm{Kr}$ & & $\mathrm{h}$ & \\
\hline & $\mathrm{Kr}$ & & $m$ & \\
\hline
\end{tabular}




\begin{tabular}{|c|c|c|c|c|}
\hline NUCLIDE & & HALF LIFE & & CURIE \\
\hline 360900 & Kr 90 & $3.232 \mathrm{E}+01$ & s & $3.750 \mathrm{E}-01$ \\
\hline 10 & $\mathrm{Kr} 91$ & $8.570 \mathrm{E}+00$ & S & $3.750 \mathrm{E}-01$ \\
\hline 920 & $\mathrm{Kr} 92$ & $1.840 \mathrm{E}+00$ & s & $3.750 \mathrm{E}-01$ \\
\hline 930 & $\mathrm{Kr} 93$ & $1.286 \mathrm{E}+00$ & 5 & $3.750 \mathrm{E}-01$ \\
\hline 360940 & $\mathrm{Kr} 94$ & $2.000 \mathrm{E}-01$ & s & $3.750 \mathrm{E}-01$ \\
\hline 360950 & $\mathrm{Kr} 95$ & $7.800 \mathrm{E}-01$ & s & $0 \mathrm{E}-01$ \\
\hline & $\mathrm{Kr} 97$ & $1.485 \mathrm{E}-01$ & s & $0 \mathrm{E}-01$ \\
\hline 980 & $\mathrm{Kr} 98$ & $2.243 \mathrm{E}-01$ & s & $3.750 \mathrm{E}-01$ \\
\hline 370 & La137 & $6.000 \mathrm{E}+04$ & $y r$ & $3.750 \mathrm{E}-01$ \\
\hline & La138 & $1.050 \mathrm{E}+11$ & $y r$ & $0 \mathrm{E}-01$ \\
\hline 00 & La140 & $1.678 \mathrm{E}+00$ & d & $0 \mathrm{E}-0$ \\
\hline 10 & La141 & $3.920 \mathrm{E}+00$ & $\mathrm{~h}$ & $0 \mathrm{E}-01$ \\
\hline & La142 & $9.110 \mathrm{E}+01$ & m & $0 \mathrm{E}-01$ \\
\hline & La143 & $1.420 \mathrm{E}+01$ & m & $0 \mathrm{E}-01$ \\
\hline 440 & La144 & $4.080 \mathrm{E}+01$ & S & 3 \\
\hline & La145 & $2.480 \mathrm{E}+01$ & s & \\
\hline 60 & La146 & $6.270 \mathrm{E}+00$ & $s$ & $3.750 \mathrm{E}-01$ \\
\hline 70 & La147 & $4.015 \mathrm{E}+00$ & s & $0 \mathrm{E}-01$ \\
\hline 0 & La148 & $1.050 \mathrm{E}+00$ & s & \\
\hline 0 & La149 & $50 \mathrm{E}+00$ & s & U1 \\
\hline & La151 & E-01 & s & \\
\hline 20 & La152 & 3.094E-01 & s & $3.750 \mathrm{E}-01$ \\
\hline 30 & La153 & $4.370 \mathrm{E}-01$ & s & E-01 \\
\hline & La154 & $1.753 \mathrm{E}-01$ & 5 & 0 \\
\hline & Lu174 & $3.310 \mathrm{E}+00$ & $y r$ & \\
\hline 41 & Lu174m & $20 \mathrm{E}+02$ & d & 3 \\
\hline 60 & Lu176 & $3.780 \mathrm{E}+10$ & $y r$ & $0 \mathrm{E}-01$ \\
\hline 71 & Lu176m & $3.635 \mathrm{E}+00$ & h & \\
\hline $7 \overline{1}$ & Lu177 & $6.734 \mathrm{E}+00$ & d & 01 \\
\hline & Lu177m & $1.604 \mathrm{E}+02$ & d & 3 \\
\hline 80 & Lu178 & $2.840 \mathrm{E}+01$ & $\mathrm{~m}$ & $0 \mathrm{E}-01$ \\
\hline 71 & Lu178m & $2.310 \mathrm{E}+01$ & $\mathrm{~m}$ & 3 \\
\hline 12 & $\operatorname{Mg} 28$ & $2.091 \mathrm{E}+01$ & $\mathrm{~h}$ & 01 \\
\hline & Mn 52 & $\mathrm{~L}+00$ & d & 3 \\
\hline & $\mathrm{Mn} 52 \mathrm{~m}$ & $\mathrm{E}+01$ & $\mathrm{~m}$ & \\
\hline & $\mathrm{Mn} 53$ & $3.740 \mathrm{E}+06$ & $y r$ & 01 \\
\hline & Mn 54 & $3.123 E+02$ & d & 3 \\
\hline & Mn 56 & $\mathrm{E}+00$ & $\vec{h}$ & \\
\hline & Mo 93 & $4.000 \mathrm{E}+03$ & $y r$ & \\
\hline & Mo $93 \mathrm{~m}$ & $6.850 \mathrm{E}+00$ & $h$ & 01 \\
\hline & Mo 99 & $6.594 \mathrm{E}+01$ & $\mathrm{~h}$ & 01 \\
\hline & Mo101 & 461E+01 & m & \\
\hline & Mo102 & $E+01$ & $\mathrm{~m}$ & 3 \\
\hline & Mo103 & $6.750 \mathrm{E}+01$ & s & \\
\hline & Mo104 & $00 \mathrm{E}+01$ & $\mathrm{~s}$ & 3 \\
\hline & Mo105 & $0 \mathrm{E}+01$ & s & \\
\hline 4 & Mo106 & 0 & s & \\
\hline & Mo107 & $E+00$ & $\mathrm{~s}$ & \\
\hline & Mo108 & $\mathrm{E}+00$ & 5 & \\
\hline & Mo1C & E- & s & \\
\hline & Mo11 & 3 & s & \\
\hline 42 & Mo11 & 6 & 5 & \\
\hline 42 & Mo11 & 1 & s & \\
\hline 4 & Mo114 & 3 & $\mathrm{~s}$ & 3 \\
\hline & $\mathrm{Na} 22$ & $02 \mathrm{E}+00$ & $y r$ & \\
\hline & $\mathrm{Na} 24$ & $E+01$ & $h$ & \\
\hline 4 & $\mathrm{Nb} 93 n$ & $E+$ & $y r$ & \\
\hline 4 & Nb 94 & 4 & $\mathrm{yr}$ & \\
\hline & $\mathrm{Nb} 94 \mathrm{~m}$ & $\mathrm{E}+00$ & $\mathrm{~m}$ & 3 \\
\hline & $\mathrm{Nb} 95$ & $E+01$ & d & $5-0$. \\
\hline & $\mathrm{Nb} 95 \mathrm{~m}$ & $\mathrm{E}+$ & $\bar{h}$ & \\
\hline & $\mathrm{Nb} 96$ & $\mathrm{E}+01$ & $\mathrm{~h}$ & \\
\hline 4 & $\mathrm{Nb} 97$ & & $\mathrm{~m}$ & \\
\hline 4 & $\mathrm{Nb} 97 \mathrm{~m}$ & $0 \mathrm{E}+$ & s & 3 \\
\hline 4 & $\mathrm{Nb} 98$ & $60 \mathrm{E}+00$ & s & JE-U \\
\hline & $\mathrm{Nb} 98$ & $\mathrm{E}+$ & $\mathrm{m}$ & 5 \\
\hline & $\mathrm{Nb} 99$ & $00 \mathrm{E}+01$ & s & \\
\hline & $\mathrm{Nb}$ & $0 \mathrm{E}+$ & $\mathrm{m}$ & L \\
\hline & $\mathrm{Nb} 100$ & $00 \mathrm{E}+$ & S & OE- \\
\hline & $\mathrm{Nb} 100 \mathrm{~m}$ & 00 & $\mathbf{s}$ & \\
\hline
\end{tabular}




\begin{tabular}{|c|c|c|c|c|}
\hline NUCLIDE & & HALF LIFE & & CURIE \\
\hline 411010 & Nb101 & $7.100 \mathrm{E}+00$ & 5 & $3.750 \mathrm{E}-01$ \\
\hline 411020 & Nb102 & $1.300 \mathrm{E}+00$ & $\mathbf{S}$ & 5E-01 \\
\hline 030 & Nb103 & $1.500 \mathrm{E}+00$ & s & $3.750 \mathrm{E}-01$ \\
\hline 40 & Nb104 & $4.800 \mathrm{E}+00$ & s & $3.750 \mathrm{E}-01$ \\
\hline 411050 & Nb105 & $2.950 \mathrm{E}+00$ & S & $3.750 \mathrm{E}-01$ \\
\hline 411060 & $\mathrm{Nb} 106$ & $1.020 \mathrm{E}+00$ & s & $0 \bar{E}-01$ \\
\hline 411070 & Nb107 & $3.300 \mathrm{E}-01$ & s & $3.750 \mathrm{E}-01$ \\
\hline 80 & Nb108 & $1.930 \mathrm{E}-01$ & $\mathrm{~s}$ & $3.750 \mathrm{E}-01$ \\
\hline 411 & Nb109 & $1.900 \mathrm{E}-01$ & s & $3.750 \mathrm{E}-01$ \\
\hline 41] & Nb111 & 1. $562 \mathrm{E}-01$ & s & 3 \\
\hline 601410 & $\mathrm{Nd} 141$ & $2.490 \mathrm{E}+00$ & $\mathrm{~h}$ & $0 \mathrm{E}-01$ \\
\hline 601440 & Nd144 & $2.290 \mathrm{E}+15$ & $y r$ & $3.750 \mathrm{E}-01$ \\
\hline 60 & $\mathrm{Nd} 147$ & $1.098 \mathrm{E}+01$ & d & $3.750 \mathrm{E}-01$ \\
\hline 601490 & $\mathrm{Nd} 149$ & $1.728 \mathrm{E}+00$ & h & $3.750 \mathrm{E}-01$ \\
\hline 601510 & Nd151 & 1. 244E+01 & m & \\
\hline 60 & $\mathrm{Nd} 152$ & 1. $140 \mathrm{E}+01$ & m & $E-01$ \\
\hline 60 & Nd153 & $3.160 \mathrm{E}+01$ & $\mathbf{s}$ & 3 \\
\hline & $\mathrm{Nd} 154$ & $2.590 \mathrm{E}+01$ & s & $0 \mathrm{E}-0$ \\
\hline 601550 & Nd155 & $8.900 \mathrm{E}+00$ & s & \\
\hline 60 & Nd156 & $5.470 \mathrm{E}+00$ & $\mathbf{S}$ & \\
\hline 60 & Nd157 & $4.150 \mathrm{E}+00$ & S & 3 \\
\hline & Nd158 & $7.890 \mathrm{E}+00$ & $\mathrm{~s}$ & \\
\hline 601 & Nd159 & $1.410 \mathrm{E}+00$ & s & 3 \\
\hline 280560 & Ni 56 & $6.077 \mathrm{E}+00$ & d & \\
\hline 70 & Ni 57 & $3.560 \mathrm{E}+01$ & $\mathrm{~h}$ & 01 \\
\hline 28 & Ni 59 & $7.600 \mathrm{E}+04$ & yr & 01 \\
\hline & Ni 63 & $1.001 \mathrm{E}+02$ & $y r$ & \\
\hline 28 & $\mathrm{Ni} 65$ & $2.517 \mathrm{E}+00$ & h & 3 \\
\hline 280 & Ni 72 & $2.100 \mathrm{E}+00$ & $\mathbf{s}$ & \\
\hline 280 & Ni 73 & $7.000 \mathrm{E}-01$ & s & 3 \\
\hline 740 & Ni 74 & $5.400 \mathrm{E}-01$ & s & 3 \\
\hline 50 & Ni 75 & $6.000 \mathrm{E}-01$ & s & 2 \\
\hline & Ni 76 & $50 \mathrm{E}-01$ & s & \\
\hline 70 & Ni 77 & 1. & s & \\
\hline 80 & Ni 78 & $1.376 \mathrm{E}-01$ & s & 01 \\
\hline 350 & Os 185 & $9.360 \mathrm{E}+01$ & d & 3 \\
\hline 891 & os $189 \mathrm{~m}$ & $5.800 \mathrm{E}+00$ & $\mathrm{~h}$ & $\perp$ \\
\hline 76 & Os190m & $300 \mathrm{E}+00$ & $\mathrm{~m}$ & \\
\hline 76 & Os191 & 1. $540 \mathrm{E}+01$ & d & 01 \\
\hline 761911 & Os191m & 1. 310E+01 & $\mathrm{h}$ & 3 \\
\hline 76 & Os193 & $3.011 \mathrm{E}+01$ & $\mathrm{~h}$ & \\
\hline 76. & Os194 & $6.000 \mathrm{E}+00$ & $y r$ & 3 \\
\hline 150320 & P 32 & $426 \mathrm{E}+01$ & d & \\
\hline 150330 & P 33 & $2.534 \mathrm{E}+01$ & d & 01 \\
\hline 822020 & Pb202 & $5.250 \mathrm{E}+04$ & $y r$ & \\
\hline & Pb203 & $5.187 \mathrm{E}+01$ & $h$ & 7 \\
\hline & Pb205 & $30 \mathrm{E}+07$ & $y r$ & \\
\hline 090 & Pb209 & $3.253 \mathrm{E}+00$ & $h$ & $\lambda_{2}$ \\
\hline 82 & $\mathrm{~Pb} 210$ & $2.230 \mathrm{E}+01$ & $y r$ & 3 \\
\hline & Pb211 & $3.610 \mathrm{E}+01$ & $\mathrm{~m}$ & \\
\hline & Pb212 & $\mathrm{E}+01$ & $\mathrm{~h}$ & \\
\hline & $\mathrm{Pb} 214$ & $\mathrm{E}+01$ & m & \\
\hline 4( & Pd103 & $1.699 \mathrm{E}+01$ & d & 3 \\
\hline & Pd10 & $6.500 \mathrm{E}+06$ & $\mathrm{yr}$ & 3 \\
\hline & $\mathrm{Pd} 10$ & $30 \mathrm{E}+01$ & $\mathrm{~s}$ & \\
\hline & Pd10 & $70 \mathrm{E}+01$ & $\mathrm{~h}$ & \\
\hline & Pd10 & $E+00$ & $\mathrm{~m}$ & \\
\hline & Pd11. & $E+01$ & m & \\
\hline & $\mathrm{Pd} 111$ & $\mathrm{~F}+00$ & $\mathrm{~h}$ & \\
\hline & $\mathrm{Pd} 1$ & 2 & $\mathrm{~h}$ & \\
\hline & Pd1] & $00 \mathrm{E}+01$ & $\mathbf{s}$ & \\
\hline & Pd1] & $E+00$ & m & \\
\hline & Pd11 & $00 \mathrm{E}+01$ & $s$ & \\
\hline 4611 & Pd1 & $00 \mathrm{E}+$ & s & \\
\hline & $\mathrm{Pd} 1$ & $n^{\circ}$ & 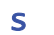 & E- \\
\hline & $\mathrm{Pd} 12$ & JUUE- & $\mathbf{s}$ & \\
\hline & Pd1 & $22 \mathrm{E}-$ & s & $0 \mathrm{E}-0$ \\
\hline & Pd1 & $00 \mathrm{E}-$ & s & \\
\hline & Pd1 & E- & $\mathbf{s}$ & \\
\hline & Pd1 & & $\mathbf{s}$ & \\
\hline
\end{tabular}




\begin{tabular}{|c|c|c|c|c|}
\hline NUCLIDE & & HALF LIFE & & CURIE \\
\hline 611430 & Pm143 & $2.650 \mathrm{E}+02$ & d & $3.750 \mathrm{E}-01$ \\
\hline 611440 & Pm144 & $3.630 \mathrm{E}+02$ & d & $50 \mathrm{E}-01$ \\
\hline 450 & Pm145 & $1.770 \mathrm{E}+01$ & yr & $0 \mathrm{E}-01$ \\
\hline 460 & Pm146 & $5.530 \mathrm{E}+00$ & $\mathrm{yr}$ & $50 \mathrm{E}-01$ \\
\hline & Pm147 & $2.623 \mathrm{E}+00$ & yr & $750 E-01$ \\
\hline 480 & $\mathrm{Pm} 148$ & $5.370 \mathrm{E}+00$ & d & $3.750 \mathrm{E}-01$ \\
\hline 481 & Pm148m & $29 \mathrm{E}+01$ & d & $0 \mathrm{E}-01$ \\
\hline 490 & Pm149 & 5. $308 \mathrm{E}+01$ & h & $0 \mathrm{E}-01$ \\
\hline 6 & Pm150 & $2.680 \mathrm{E}+00$ & $\mathrm{~h}$ & $0 \mathrm{E}-01$ \\
\hline 510 & Pm151 & $2.840 \mathrm{E}+01$ & $\mathrm{~h}$ & $50 \mathrm{E}-01$ \\
\hline 20 & Pm152 & $4.120 \mathrm{E}+00$ & m & $3.750 \mathrm{E}-01$ \\
\hline 21 & Pm152m & $0 \mathrm{E}+00$ & m & 01 \\
\hline 530 & Pm153 & $5.250 \mathrm{E}+00$ & m & 0E-01 \\
\hline & Pm154 & $30 E+00$ & m & $0 \mathrm{E}-01$ \\
\hline 41 & Pm154m & $2.680 \mathrm{E}+00$ & m & $0 \mathrm{E}-01$ \\
\hline 50 & Pm155 & $4.150 \mathrm{E}+01$ & $\mathbf{s}$ & \\
\hline 60 & Pm156 & $2.670 \mathrm{E}+01$ & s & $E-01$ \\
\hline 61 & Pm157 & $1.056 \mathrm{E}+01$ & $\mathbf{s}$ & E-01 \\
\hline & Pm158 & $4.800 \mathrm{E}+00$ & s & $0 E-01$ \\
\hline 590 & Pm159 & $4.230 \mathrm{E}+00$ & $s$ & $E-01$ \\
\hline 0 & Pr142 & 1. $912 \mathrm{E}+01$ & h & -01 \\
\hline 5 & $\operatorname{Pr} 143$ & $1.357 \mathrm{E}+01$ & d & E-01 \\
\hline 5 & Pr144 & $3 \mathrm{E}+01$ & $\mathrm{~m}$ & -01 \\
\hline 41 & Pr144m & $7.200 \mathrm{E}+00$ & m & $750 \mathrm{E}-01$ \\
\hline 50 & Pr145 & $5.984 E+00$ & $\mathrm{~h}$ & $E-01$ \\
\hline 5 & Pr146 & $2.415 \mathrm{E}+01$ & $\mathrm{~m}$ & \\
\hline 5 & $\operatorname{Pr} 147$ & 1. 340E +01 & m & 01 \\
\hline 400 & Pr148 & $70 \mathrm{E}+00$ & $\mathrm{~m}$ & \\
\hline 90 & Pr149 & $2.260 \mathrm{E}+00$ & m & 01 \\
\hline 5 & Pr151 & $0 \mathrm{E}+01$ & S & \\
\hline 0 & Pr152 & $0 \mathrm{E}+00$ & $\mathbf{s}$ & 01 \\
\hline 53 & Pr153 & 4. $300 \mathrm{E}+00$ & $s$ & -01 \\
\hline 40 & Pr154 & $2.300 \mathrm{E}+00$ & $\mathbf{s}$ & 01 \\
\hline & Pr155 & $0 \mathrm{E}+00$ & $\mathbf{s}$ & \\
\hline 0 & Pr1 & 4E-01 & 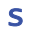 & \\
\hline ○ & Pr157 & $6.780 \mathrm{E}-01$ & $\mathbf{s}$ & 01 \\
\hline 58 & Pr158 & $2.630 \mathrm{E}-01$ & 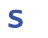 & -01 \\
\hline & Pr1 & OE-01 & s & 01 \\
\hline & Pt1 & $02 \mathrm{E}+00$ & d & 01 \\
\hline 930 & Pt1S & $5.000 \mathrm{E}+01$ & $y r$ & 01 \\
\hline 31 & Pt193m & $4.330 \mathrm{E}+00$ & d & 01 \\
\hline & Pt195m & $4.020 \mathrm{E}+00$ & d & \\
\hline 170 & Pt197 & $89 \mathrm{E}+01$ & $\mathrm{~h}$ & -01 \\
\hline 971 & Pt197m & $9.541 \mathrm{E}+01$ & $\mathrm{~m}$ & \\
\hline 10 & Rb 81 & 4. & $\mathrm{~h}$ & \\
\hline$?$ & $\mathrm{Rb} 82$ & 1.273E+ & $\mathrm{m}$ & 01 \\
\hline & $\mathrm{Rb} 83$ & $8.620 \mathrm{E}+01$ & d & \\
\hline & $\mathrm{Rb} 84$ & $3.277 \mathrm{E}+01$ & d & 01 \\
\hline & $\mathrm{Rb} 86$ & 1. & d & \\
\hline & $\mathrm{Rb} 87$ & $4.750 \mathrm{E}+10$ & $\mathrm{yr}$ & 01 \\
\hline & $\mathrm{Rb} 88$ & $E+01$ & m & 01 \\
\hline & $\mathrm{Rb} 89$ & $5 E+01$ & $\mathrm{~m}$ & 01 \\
\hline & $\mathrm{Rb} 90$ & 2 & $\mathbf{s}$ & \\
\hline & $\mathrm{Rb} 90 \mathrm{~m}$ & & $\mathbf{s}$ & \\
\hline & $\mathrm{Rb} 91$ & 5. & $\mathbf{s}$ & \\
\hline & $\mathrm{Rb} 92$ & 4. & $\mathbf{s}$ & \\
\hline & $\mathrm{Rb} 93$ & 5 & $\mathbf{s}$ & \\
\hline & $\mathrm{Rb} 94$ & & $\mathbf{s}$ & \\
\hline & $\mathrm{Rb} 95$ & & $s$ & \\
\hline & $\mathrm{Rb} 97$ & & 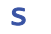 & \\
\hline & $\mathrm{Rb} 98$ & 1. & $\mathbf{s}$ & \\
\hline & $\mathrm{Rb}$ & & $\mathrm{s}$ & \\
\hline & $\mathrm{Rb} 100$ & LOOE-02 & $s$ & \\
\hline & Re182a & 1. $270 \mathrm{E}+01$ & $\mathrm{~h}$ & 01 \\
\hline & Re182b & $6.400 \mathrm{E}+01$ & $\mathrm{~h}$ & \\
\hline & Re184 & 3. & d & \\
\hline & Re184m & & d & \\
\hline & Re18 & 3. & d & E-01 \\
\hline & Re18 & $00 \mathrm{E}+$ & $\mathrm{yr}$ & 01 \\
\hline & $\operatorname{Re} 18$ & & yr & \\
\hline
\end{tabular}




\begin{tabular}{|c|c|c|c|c|}
\hline NUCLIDE & & HALF LIFE & & CURIE \\
\hline 751880 & Re188 & $1.700 \mathrm{E}+01$ & $\mathrm{~h}$ & $3.750 \mathrm{E}-01$ \\
\hline 751881 & Re188m & $1.860 \mathrm{E}+01$ & $\mathrm{~m}$ & $3.750 \mathrm{E}-01$ \\
\hline 751890 & Re189 & $2.430 \mathrm{E}+01$ & $\mathrm{~h}$ & $3.750 \mathrm{E}-01$ \\
\hline 451010 & Rh101 & $3.300 \mathrm{E}+00$ & $\mathrm{yr}$ & $3.750 \mathrm{E}-01$ \\
\hline 011 & $\mathrm{Rh} 101 \mathrm{~m}$ & $4.340 \mathrm{E}+00$ & d & $3.750 \mathrm{E}-01$ \\
\hline 020 & Rh102 & $2.070 \mathrm{E}+02$ & d & $3.750 \mathrm{E}-01$ \\
\hline 021 & Rh102m & $2.900 \mathrm{E}+00$ & $y r$ & $3.750 \mathrm{E}-01$ \\
\hline 031 & Rh103m & $5.612 \mathrm{E}+01$ & $\mathrm{~m}$ & $3.750 \mathrm{E}-01$ \\
\hline 45 & Rh105 & $3.536 \mathrm{E}+01$ & $\mathrm{~h}$ & $3.750 \mathrm{E}-01$ \\
\hline & Rh105m & $4.500 \mathrm{E}+01$ & $\mathrm{~s}$ & $0 \mathrm{E}-0$ \\
\hline & Rh106 & $2.980 \mathrm{E}+01$ & $\mathrm{~s}$ & 01 \\
\hline & Rh106m & $310 \mathrm{E}+02$ & $\mathrm{~m}$ & $0 \mathrm{E}-01$ \\
\hline 45 & Rh107 & $2.170 \mathrm{E}+01$ & $\mathrm{~m}$ & $3.750 \mathrm{E}-01$ \\
\hline 80 & Rh108 & $1.680 \mathrm{E}+01$ & s & $0 \mathrm{E}-01$ \\
\hline 81 & Rh108m & $6.000 \mathrm{E}+00$ & m & 3 \\
\hline & Rh109 & $8.000 E+01$ & s & 3 \\
\hline & Rh109m & $5.000 \mathrm{E}+01$ & $\mathrm{~s}$ & $3.750 \mathrm{E}-01$ \\
\hline 45 & Rh111 & $1.100 \mathrm{E}+01$ & s & $E-01$ \\
\hline & Rh112 & $2.100 \mathrm{E}+00$ & $\mathrm{~s}$ & \\
\hline & Rh113 & $2.800 \mathrm{E}+00$ & s & 3 \\
\hline & Rh114 & $1.850 \mathrm{E}+00$ & $\mathrm{~s}$ & \\
\hline 45 & Rh118 & $2.953 \mathrm{E}-01$ & $\mathrm{~s}$ & $U$ E -01 \\
\hline 45 & Rh119 & $4.478 \mathrm{E}-01$ & s & 3 \\
\hline 45 & Rh120 & $1.624 \mathrm{E}-01$ & $\mathrm{~s}$ & 01 \\
\hline 45 & Rh121 & $210 \mathrm{E}-01$ & s & \\
\hline 30 & Rh123 & 1 & $\mathrm{~s}$ & 3 \\
\hline 44 & Ru 97 & $2.900 \mathrm{E}+00$ & d & 3 \\
\hline 30 & Ru103 & $3.926 \mathrm{E}+01$ & $\vec{d}$ & \\
\hline 50 & Ru105 & $4.440 \mathrm{E}+00$ & $\mathrm{~h}$ & 3 \\
\hline 44 & Ru106 & $3.736 \mathrm{E}+02$ & d & 3 \\
\hline 44 & Ru108 & $50 \mathrm{E}+00$ & $\mathrm{~m}$ & 3 \\
\hline 441090 & Ru109 & $450 \mathrm{E}+01$ & $s$ & \\
\hline 44 & Ru111 & $20 \mathrm{E}+$ & $\mathrm{m}$ & \\
\hline 44 & Ru112 & $1.750 \mathrm{E}+00$ & s & \\
\hline 44 & Ru113 & $00 \mathrm{E}-01$ & $\mathrm{~s}$ & \\
\hline 441 & Ru114 & 00E-01 & 5 & \\
\hline 441180 & Ru118 & $60 \mathrm{E}-01$ & $\mathrm{~s}$ & \\
\hline 44 & Ru120 & $32 \mathrm{E}-01$ & $\mathrm{~s}$ & 3 \\
\hline 16 & 535 & $8.732 \mathrm{E}+01$ & $d$ & 3 \\
\hline 511170 & sb117 & $2.800 \mathrm{E}+00$ & $\mathrm{~h}$ & 01 \\
\hline 511203 & sb120b & $5.760 \mathrm{E}+00$ & d & 01 \\
\hline 511220 & Sb122 & $2.724 \mathrm{E}+00$ & $d$ & 01 \\
\hline 51124 & Sb124 & $6.020 \mathrm{E}+01$ & d & 3 \\
\hline 511250 & sb125 & $2.758 \mathrm{E}+00$ & $y r$ & \\
\hline 260 & Sb126 & $1.246 \mathrm{E}+01$ & d & 3 \\
\hline 511261 & sb126m & $1.915 \mathrm{E}+01$ & $\mathrm{~m}$ & \\
\hline 51127 & Sb127 & 0 & $\ddot{d}$ & \\
\hline 280 & Sb128 & $10 \mathrm{E}+00$ & $\bar{h}$ & 3 \\
\hline 81 & $\mathrm{sb} 128 \mathrm{~m}$ & $40 \mathrm{E}+01$ & $\mathrm{~m}$ & \\
\hline 90 & Sb129 & $4.400 \mathrm{E}+00$ & $\mathrm{~h}$ & \\
\hline 300 & sb130 & $50 \mathrm{E}+01$ & $\mathrm{~m}$ & \\
\hline 51 & $\mathrm{sb} 130 \mathrm{~m}$ & $00 \mathrm{E}+00$ & $\mathrm{~m}$ & \\
\hline 5$]$ & Sb131 & ? & $\mathrm{m}$ & \\
\hline 5$]$ & Sb132 & $90 \mathrm{E}+00$ & $\mathrm{~m}$ & \\
\hline & Sb132m & $00 \mathrm{E}+00$ & m & \\
\hline 51 & sb133 & $0 \mathrm{E}+$ & $m$ & \\
\hline & Sb134 & $\mathrm{E}-01$ & s & \\
\hline 5 & Sb134m & 1 & $\mathrm{~s}$ & \\
\hline & sb135 & $10 \mathrm{E}+00$ & $\mathrm{~s}$ & \\
\hline & Sb136 & E- & $\mathrm{s}$ & \\
\hline & sb137 & & $\mathrm{s}$ & \\
\hline & sb138 & & $\mathrm{s}$ & \\
\hline & sb139 & & $\mathrm{s}$ & 3 \\
\hline & SC 44 & 7E+ & $\hat{h}$ & 3 \\
\hline & Sc $44 m$ & $50 \mathrm{E}+01$ & $\mathrm{~h}$ & J-U \\
\hline & SC 46 & L. & d & 5 \\
\hline & Sc 47 & $49 \mathrm{E}+$ & d & \\
\hline & SC 48 & & $\mathrm{~h}$ & E- \\
\hline & SC 49 & $20 \mathrm{E}+$ & m & JE- \\
\hline & Se 73 & 50 & $\mathrm{~h}$ & \\
\hline
\end{tabular}




\begin{tabular}{|c|c|c|c|c|}
\hline NUCLIDE & & HALF LIFE & & CURIE \\
\hline 750 & se 75 & $1.198 \mathrm{E}+02$ & d & $3.750 \mathrm{E}-01$ \\
\hline 790 & se 79 & $1.130 \mathrm{E}+06$ & $y r$ & $0 \mathrm{E}-01$ \\
\hline 340791 & se $79 m$ & $3.920 \mathrm{E}+00$ & $\mathrm{~m}$ & $3.750 \mathrm{E}-01$ \\
\hline 340810 & Se 81 & $1.845 \mathrm{E}+01$ & m & $3.750 \mathrm{E}-01$ \\
\hline 340811 & Se $81 \mathrm{~m}$ & $5.728 \mathrm{E}+01$ & m & $0 \bar{E}-01$ \\
\hline 830 & Se $8 \overline{3}$ & $2.230 \mathrm{E}+01$ & m & $0 \mathrm{E}-01$ \\
\hline & Se $83 \mathrm{~m}$ & $7.010 \mathrm{E}+01$ & s & 0 \\
\hline 340 & Se 84 & $3.100 \mathrm{E}+00$ & m & $3.750 \mathrm{E}-01$ \\
\hline 350 & Se 85 & $3.170 \mathrm{E}+01$ & s & $0 E-01$ \\
\hline 351 & Se $85 \mathrm{~m}$ & $1.900 \mathrm{E}+01$ & s & \\
\hline 860 & Se 86 & $0 \mathrm{E}+01$ & s & 3 \\
\hline 370 & Se 87 & $\mathrm{E}+00$ & $\mathrm{~s}$ & \\
\hline 380 & Se 88 & $1.530 \mathrm{E}+00$ & $\mathrm{~s}$ & UE-UI \\
\hline 390 & Se 89 & $4.100 \mathrm{E}-01$ & $\mathbf{s}$ & \\
\hline 900 & Se 90 & $5 E-01$ & s & 3 \\
\hline 910 & se 91 & $2.700 \mathrm{E}-01$ & S & 3 \\
\hline 20 & Se 92 & $2.478 \mathrm{E}-01$ & 5 & \\
\hline 310 & si 31 & $1.573 \mathrm{E}+02$ & m & $5-U \perp$ \\
\hline 160 & Sm146 & $1.030 \mathrm{E}+08$ & $y r$ & \\
\hline 70 & Sm147 & $60 \mathrm{E}+11$ & $y r$ & 01 \\
\hline 480 & Sm148 & $00 \mathrm{E}+15$ & yr & 3 \\
\hline 90 & Sm149 & $00 \mathrm{E}+15$ & $\mathrm{yr}$ & 3 \\
\hline 621510 & Sm151 & $9.000 \mathrm{E}+01$ & $y r$ & 3 \\
\hline 30 & Sm153 & $4.628 \mathrm{E}+01$ & $h$ & 3 \\
\hline 62 & Sm155 & $\mathrm{E}+01$ & $\mathrm{~m}$ & 3 \\
\hline 6 & Sm156 & $00 \mathrm{E}+00$ & $\mathrm{~h}$ & \\
\hline 570 & Sm157 & $320 \mathrm{E}+02$ & s & 3 \\
\hline 621580 & Sm158 & $5.300 \mathrm{E}+00$ & $\mathrm{~m}$ & \\
\hline 90 & Sm159 & $37 E+01$ & $\mathbf{s}$ & 3 \\
\hline 100 & Sn110 & $4.110 \mathrm{E}+00$ & h & \\
\hline 130 & Sn113 & $1.151 \mathrm{E}+02$ & d & 3 \\
\hline 501171 & Sn117m & $1.360 \mathrm{E}+01$ & d & UI \\
\hline 191 & Sn119m & $\mathrm{LE}+02$ & d & 0 \\
\hline 210 & Sn121 & $\mathrm{E}+01$ & $\mathrm{~h}$ & 3 \\
\hline 50 & Sn121m & $\mathrm{E}+01$ & $y r$ & \\
\hline 230 & Sn123 & $92 \mathrm{E}+02$ & d & 01 \\
\hline 501231 & Sn123m & $4.006 \mathrm{E}+01$ & m & \\
\hline 50 & Sn125 & $40 \mathrm{E}+00$ & d & 3 \\
\hline 50 & $\mathrm{Sn} 125 \mathrm{~m}$ & $9.520 \mathrm{E}+00$ & $\mathrm{~m}$ & 3 \\
\hline 50 & Sn126 & $00 \mathrm{E}+05$ & $y r$ & \\
\hline 270 & Sn127 & $2.100 \mathrm{E}+00$ & $h$ & )1 \\
\hline 501271 & Sn127m & $4.130 \mathrm{E}+00$ & m & \\
\hline 50 & Sn12 & $E+01$ & $\mathrm{~m}$ & \\
\hline 50 & Sn1 & $E+00$ & m & \\
\hline 291 & Sn129m & $6.900 \mathrm{E}+00$ & $\mathrm{~m}$ & 3 \\
\hline 300 & Sn130 & $20 \mathrm{E}+00$ & m & 3 \\
\hline 50 & Sn131 & $E+01$ & s & \\
\hline & Sn1 & 3. & $\mathrm{~s}$ & \\
\hline & Sn133 & $E+00$ & $\mathrm{~s}$ & \\
\hline & sn13 & $\mathrm{E}+00$ & s & \\
\hline & Sn13 & 2 & $\mathrm{~s}$ & \\
\hline 360 & Sn136 & -01 & $\mathrm{~s}$ & \\
\hline $3 \varepsilon$ & Sr 82 & $=01$ & d & \\
\hline & Sr 85 & $E+01$ & d & 3 \\
\hline & $\mathrm{Sr} 85 \mathrm{~m}$ & $\mathrm{E}+01$ & m & \\
\hline & $\mathrm{sr} 87 \mathrm{~m}$ & & $\hat{h}$ & \\
\hline & Sr 89 & 1 & d & \\
\hline & sr 90 & & $y r$ & \\
\hline & sr 91 & $\mathrm{E}+$ & $h$ & 3 \\
\hline & sr 92 & $E+00$ & $\mathrm{~h}$ & \\
\hline & Sr 93 & & $\mathrm{~m}$ & \\
\hline & sr 94 & $=01$ & s & \\
\hline & Sr 95 & & $\mathbf{s}$ & \\
\hline 38 & Sr 97 & 4 & 5 & E- \\
\hline & $\mathrm{Sr}$ & UE- & s & \\
\hline & Sr 99 & $90 \mathrm{E}-$ & s & UL- \\
\hline & & 50 & $\mathrm{~s}$ & \\
\hline & Sr101 & - & $\mathrm{s}$ & \\
\hline & sr102 & E- & $\mathrm{s}$ & $E-$ \\
\hline & sr10 & -01 & s & \\
\hline
\end{tabular}




\begin{tabular}{|c|c|c|c|c|}
\hline NUCLIDE & & HALF LIFE & & CURIE \\
\hline 381040 & Sr104 & $1.925 \mathrm{E}-01$ & $\mathbf{S}$ & $3.750 \mathrm{E}-01$ \\
\hline 790 & Ta179 & $1.820 \mathrm{E}+00$ & $y r$ & $50 \mathrm{E}-01$ \\
\hline 301 & Ta180m & $1.200 \mathrm{E}+15$ & $y r$ & $50 \mathrm{E}-01$ \\
\hline 820 & Ta182 & $1.144 \mathrm{E}+02$ & d & $50 \mathrm{E}-01$ \\
\hline 321 & Ta182m & $1.584 \mathrm{E}+01$ & $\mathrm{~m}$ & $750 \mathrm{E}-01$ \\
\hline 330 & Ta183 & $5.100 \mathrm{E}+00$ & d & $3.750 \mathrm{E}-01$ \\
\hline 340 & Ta184 & $8.700 \mathrm{E}+00$ & $\mathrm{~h}$ & $0 \mathrm{E}-01$ \\
\hline 50 & Ta185 & 4. $940 \mathrm{E}+01$ & m & $0 E-01$ \\
\hline & Ta186 & $1.050 \mathrm{E}+01$ & m & $0 \mathrm{E}-01$ \\
\hline 70 & Tb157 & $7.100 \mathrm{E}+01$ & $y r$ & $750 \mathrm{E}-01$ \\
\hline 80 & Tb158 & $1.800 \mathrm{E}+02$ & $y r$ & $0 \mathrm{E}-01$ \\
\hline 00 & Tb160 & $7.230 \mathrm{E}+01$ & & $0 \mathrm{E}-01$ \\
\hline & Tb161 & $6.880 \mathrm{E}+00$ & d & $0 \mathrm{E}-01$ \\
\hline & Tс 96 & $4.280 E+00$ & d & $750 \mathrm{E}-01$ \\
\hline 61 & TC $96 \mathrm{~m}$ & $5.150 \mathrm{E}+01$ & $\mathrm{~m}$ & $3.750 \mathrm{E}-01$ \\
\hline & TC 97 & $00 \mathrm{E}+06$ & $y r$ & 01 \\
\hline & TC $97 \mathrm{~m}$ & $9.010 \mathrm{E}+01$ & d & 01 \\
\hline & Tс 98 & $4.200 \mathrm{E}+06$ & $y r$ & 01 \\
\hline 90 & Tс 99 & $2.111 \mathrm{E}+05$ & $y r$ & $0 E-01$ \\
\hline 91 & TC $99 m$ & $6.010 \mathrm{E}+00$ & $\mathrm{~h}$ & -01 \\
\hline 10 & Tc101 & $22 \mathrm{E}$ & $\mathrm{m}$ & 01 \\
\hline 20 & TC102 & $5.280 \mathrm{E}+00$ & S & 01 \\
\hline & Tc102m & $4.350 \mathrm{E}+00$ & $\mathrm{~m}$ & \\
\hline 30 & Tc103 & $5.420 \mathrm{E}+01$ & S & 01 \\
\hline 40 & Tc104 & $30 \mathrm{E}+01$ & $\mathrm{~m}$ & \\
\hline 50 & Tc105 & $7.600 \mathrm{E}+00$ & m & 01 \\
\hline 4 & Tc106 & 3. 560E+01 & S & 01 \\
\hline & Tc107 & $2.120 \mathrm{E}+01$ & s & 01 \\
\hline 80 & Tc108 & $0 \mathrm{E}+00$ & s & \\
\hline 90 & Tc109 & 8. & $\mathbf{s}$ & \\
\hline 110 & Tc111 & $0 E-01$ & S & 01 \\
\hline 12 & Tc112 & $2.800 \mathrm{E}-01$ & $\mathrm{~s}$ & 01 \\
\hline L130 & Tc113 & $1.300 \mathrm{E}-01$ & s & 01 \\
\hline 140 & Tc114 & 1.734E-01 & $\mathrm{s}$ & \\
\hline 210 & Te121 & $78 \mathrm{E}+01$ & d & 01 \\
\hline 211 & Te121m & 1. 540E+02 & d & $E-01$ \\
\hline 52 & Te123 & $00 E+13$ & $y r$ & \\
\hline 31 & Te123m & $1.197 \mathrm{E}+02$ & d & 01 \\
\hline 251 & Te125m & $5.740 \mathrm{E}+01$ & d & \\
\hline 770 & Te127 & $0 \mathrm{E}+00$ & $\mathrm{~h}$ & 01 \\
\hline 271 & Te127m & $1.090 \mathrm{E}+02$ & d & -01 \\
\hline 290 & Te129 & $6.960 \mathrm{E}+01$ & $\mathrm{~m}$ & 01 \\
\hline 91 & Te129m & $60 \mathrm{E}+01$ & d & 01 \\
\hline 310 & Te131 & $\mathrm{E}+01$ & $\mathrm{~m}$ & \\
\hline 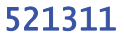 & Te131m & $3.000 \mathrm{E}+01$ & $\mathrm{~h}$ & 01 \\
\hline & Te132 & $3.204 \mathrm{E}+00$ & d & \\
\hline 0 & Te13 & $50 E+01$ & $\mathrm{~m}$ & 01 \\
\hline & Te133m & $5.540 \mathrm{E}+01$ & $\mathrm{~m}$ & \\
\hline 340 & Te134 & $\mathrm{E}+01$ & $\mathrm{~m}$ & \\
\hline 35 & Te135 & 00E+01 & S & $=-0$ \\
\hline & Te136 & $1.750 \mathrm{E}+01$ & s & \\
\hline & Te137 & $E+00$ & s & 01 \\
\hline 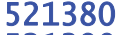 & Te1 & 1. & $s$ & \\
\hline & Te1 & 4. & s & \\
\hline & Te140 & 7. & s & \\
\hline & Te1 & 2 & $s$ & \\
\hline & Te142 & 4 & s & \\
\hline & Ti 44 & 6. & $y r$ & \\
\hline & Ti 45 & 1. & $\mathrm{~m}$ & \\
\hline & & & $\mathrm{m}$ & \\
\hline & T1200 & $10 \mathrm{E}+01$ & $\mathrm{~h}$ & \\
\hline & T1201 & $1 E+01$ & $\mathrm{~h}$ & -01 \\
\hline & T1202 & $3 E+01$ & d & \\
\hline & T1204 & DE+ & $y$ & \\
\hline & T1206 & & $\mathrm{m}$ & \\
\hline & T1207 & OEt & $\mathrm{m}$ & \\
\hline & T1208 & $53 \mathrm{E}+00$ & m & -01 \\
\hline & & ח & $\mathrm{m}$ & \\
\hline & & & m & \\
\hline & & & d & \\
\hline
\end{tabular}




\begin{tabular}{|c|c|c|c|c|}
\hline NUCLIDE & & HALF LIFE & & CURIE \\
\hline 691710 & Tm171 & $1.920 \mathrm{E}+00$ & yr & $3.750 \mathrm{E}-01$ \\
\hline & V 48 & $1.597 \mathrm{E}+01$ & d & $3.750 \mathrm{E}-01$ \\
\hline & V 49 & $3.300 \mathrm{E}+02$ & d & $750 \mathrm{E}-01$ \\
\hline & W179 & $3.705 \mathrm{E}+01$ & m & $3.750 \mathrm{E}-01$ \\
\hline 741810 & W181 & $1.212 \mathrm{E}+02$ & d & $3.750 \mathrm{E}-01$ \\
\hline 350 & W185 & $7.510 \mathrm{E}+01$ & d & $3.750 \mathrm{E}-01$ \\
\hline 870 & W187 & $2.372 \mathrm{E}+01$ & $\mathrm{~h}$ & $3.750 \mathrm{E}-01$ \\
\hline & W188 & $6.940 \mathrm{E}+01$ & d & $3.750 \mathrm{E}-01$ \\
\hline 54 & Xe122 & $2.010 E+01$ & $\mathrm{~h}$ & $3.750 \mathrm{E}-01$ \\
\hline 230 & Xe123 & $2.080 \mathrm{E}+00$ & $\mathrm{~h}$ & $3.750 \mathrm{E}-01$ \\
\hline 50 & xe125 & $1.690 \mathrm{E}+01$ & h & $3.750 \mathrm{E}-01$ \\
\hline 54 & xe127 & $3.640 \mathrm{E}+01$ & d & $3.750 \mathrm{E}-01$ \\
\hline 291 & Xe129m & $8.880 \mathrm{E}+00$ & d & $3.750 \mathrm{E}-01$ \\
\hline 311 & Xe131m & 1. 184E+01 & d & $3.750 \mathrm{E}-01$ \\
\hline 30 & Xe133 & $5.243 \mathrm{E}+00$ & d & $\mathrm{E}-01$ \\
\hline 31 & xe133m & $2.190 \mathrm{E}+00$ & $\bar{d}$ & $0 \mathrm{E}-01$ \\
\hline & Xe134m & $2.900 \mathrm{E}-01$ & s & -01 \\
\hline & Xe135 & $9.140 \mathrm{E}+00$ & $\mathrm{~h}$ & $3.750 \mathrm{E}-01$ \\
\hline & xe135m & $1.529 \mathrm{E}+01$ & m & $0 \mathrm{E}-01$ \\
\hline 54 & Xe137 & $3.818 \mathrm{E}+00$ & m & 01 \\
\hline 30 & Xe138 & $1.408 \mathrm{E}+01$ & $\mathrm{~m}$ & 01 \\
\hline & xe139 & $3.968 \mathrm{E}+01$ & s & \\
\hline 54 & Xe140 & 1. $360 \mathrm{E}+01$ & $\mathrm{~s}$ & $0 \mathrm{E}-0$ \\
\hline 54 & Xe141 & $1.730 \mathrm{E}+00$ & s & \\
\hline 5 & Xe142 & $1.220 \mathrm{E}+00$ & s & 3 \\
\hline 5 & Xe143 & $3.000 \mathrm{E}-01$ & S & 01 \\
\hline & Xe144 & $1.150 \mathrm{E}+00$ & $\mathrm{~s}$ & \\
\hline 50 & Xe145 & $9.000 \mathrm{E}-01$ & $\mathrm{~s}$ & 3 \\
\hline 54 & Xe146 & $372 E-01$ & $\mathbf{s}$ & \\
\hline 70 & Xe147 & $2.638 \mathrm{E}-01$ & $\mathrm{~s}$ & 3 \\
\hline & Y 86 & $1.474 \mathrm{E}+01$ & $\mathrm{~h}$ & 3 \\
\hline 39 & Y 87 & $7.980 \mathrm{E}+01$ & $\mathrm{~h}$ & $0 \mathrm{E}-01$ \\
\hline 39 & Y 88 & $1.067 \mathrm{E}+02$ & d & 01 \\
\hline 39 & Y 90 & $6.400 \mathrm{E}+01$ & $\bar{h}$ & 01 \\
\hline 39 & Y $90 \mathrm{~m}$ & $3.190 \mathrm{E}+00$ & $\mathrm{~h}$ & 01 \\
\hline & Y 91 & $5.851 E+01$ & d & 3 \\
\hline 39 & Y $91 \mathrm{~m}$ & $4.971 \mathrm{E}+01$ & $\mathrm{~m}$ & 01 \\
\hline 39 & Y 92 & 3. $540 \mathrm{E}+00$ & $\mathrm{~h}$ & \\
\hline 39 & Y 93 & $1.018 \mathrm{E}+01$ & $\mathrm{~h}$ & 01 \\
\hline 3 & Y 94 & $1.870 \mathrm{E}+01$ & m & 01 \\
\hline 3 & Y 95 & $1.030 \mathrm{E}+01$ & $\mathrm{~m}$ & 3 \\
\hline & Y 97 & $3.750 \mathrm{E}+00$ & $\mathbf{s}$ & 3 \\
\hline 39 & Y 98 & $480 \mathrm{E}-01$ & s & \\
\hline 39 & Y 99 & $1.470 \mathrm{E}+00$ & $s$ & 01 \\
\hline & Y100 & $7.350 \mathrm{E}-01$ & $\mathbf{s}$ & \\
\hline & Y101 & $4.480 \mathrm{E}-01$ & s & 3 \\
\hline & Y102 & $00 \mathrm{E}-01$ & $\mathbf{s}$ & \\
\hline 3 & Y10 & 2 & $\mathbf{s}$ & \\
\hline & Y1 & $442 \mathrm{E}-01$ & s & 3 \\
\hline & Y105 & $36 \mathrm{E}-01$ & s & \\
\hline & Y10 & $46 \mathrm{E}$ & s & \\
\hline & Yb1 & 1 & d & \\
\hline 70 & Yb175 & $\mathrm{E}+00$ & d & 3 \\
\hline 30 & $\mathrm{Zn} 62$ & $86 E+00$ & $\mathrm{~h}$ & 3 \\
\hline & $\mathrm{Zn} 63$ & $47 \mathrm{E}+01$ & m & \\
\hline & Zn 65 & $\mathrm{E}+02$ & d & 3 \\
\hline & $\mathrm{Zn} 69$ & 5 & m & \\
\hline & $\mathrm{Zn} 69 n$ & +01 & $\mathrm{~h}$ & \\
\hline & $\mathrm{Zn} 71 \mathrm{~m}$ & + & $\mathrm{h}$ & \\
\hline & $\mathrm{Zn} 72$ & 4 & $\mathrm{~h}$ & 3 \\
\hline & Zn 73 & $E+01$ & s & \\
\hline & $\mathrm{Zn} 74$ & ᄂ. & $s$ & \\
\hline 3 & Zn 75 & $E+$ & $\mathbf{s}$ & \\
\hline 80 & Zn 76 & $0 \mathrm{E}+00$ & s & \\
\hline 3( & Zn 77 & $80 \mathrm{E}+$ & s & $\mathrm{E}-$ \\
\hline 3 & Zn 78 & $70 \mathrm{E}+$ & s & \\
\hline & Zn 79 & $50 \mathrm{E}-01$ & s & - \\
\hline & $\mathrm{Zn} 80$ & $50 \mathrm{E}-$ & s & \\
\hline & Zn 8 & $\mathrm{E}-$ & s & $E-0$ \\
\hline & $\mathrm{Zn}$ & & s & \\
\hline
\end{tabular}




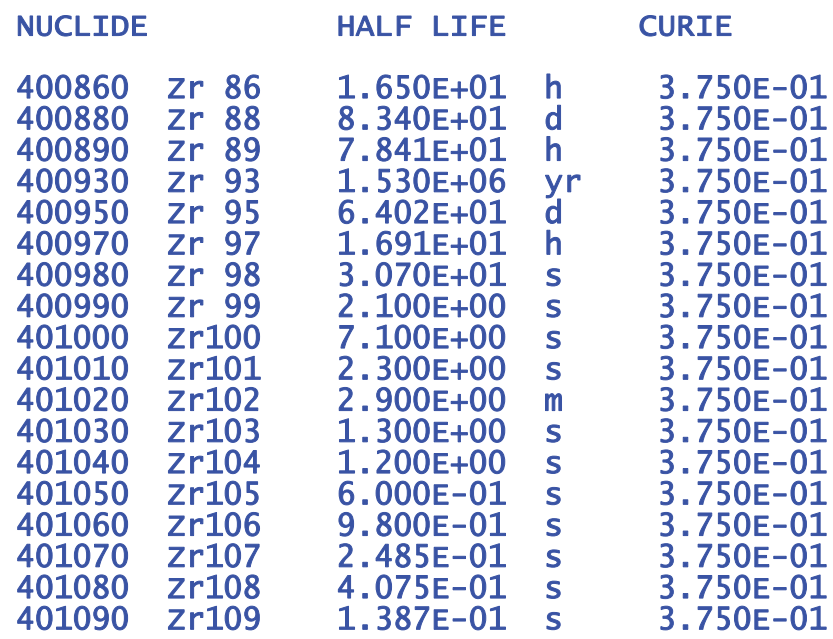

$* * *$ DIRECT RADIONUCLIDE INPUT PREVIOUS INVENTORY INCREASED BY THE FOLLOWING VALUES NUCLIDE HALF LIFE GRAM CURIE

902320 Th232 1.405E+10 yr $2.000 \mathrm{E}+032.193 \mathrm{E}-04$

$922330 \mathrm{U} 233$ 1.592E+05 yr $0.000 \mathrm{E}+000.000 \mathrm{E}+00$ WARNING-LESS THAN 1 ATOM

$922350 \mathrm{U} 235 \quad 7.038 \mathrm{E}+08$ yr $\quad 5.000 \mathrm{E}+00 \quad 1.081 \mathrm{E}-05$

922380 U238 $4.468 \mathrm{E}+09$ yr $2.040 \mathrm{E}+04 \quad 6.858 \mathrm{E}-03$

942390 Pu239 2.411E+04 yr 1.100E-01 6.824E-03

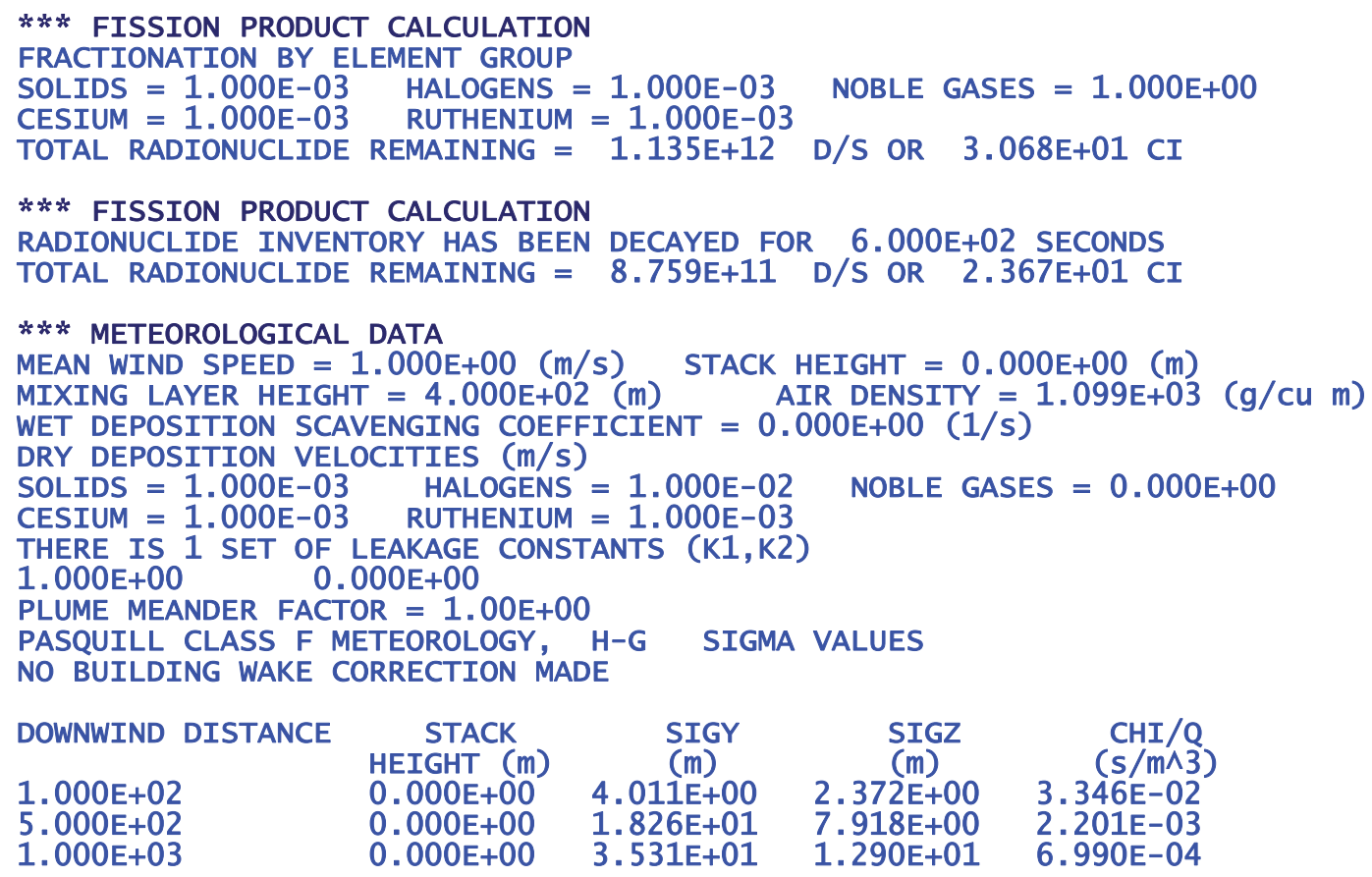

PLUME DEPLETION BY FALLOUT IS INCLUDED

FRACTION OF PLUME REMAINING AIRBORNE FOLLOWING DEPLETION BY DEPOSITION

DOWNWIND DISTANCE SOLIDS HALOGENS CESIUM RUTHENIUM

$\begin{array}{lllll}1.000 \mathrm{E}+02 & 9.480 \mathrm{E}-01 & 5.862 \mathrm{E}-01 & 9.480 \mathrm{E}-01 & 9.480 \mathrm{E}-01 \\ 5.000 \mathrm{E}+02 & 9.451 \mathrm{E}-01 & 5.684 \mathrm{E}-01 & 9.451 \mathrm{E}-01 & 9.451 \mathrm{E}-01 \\ 1.000 \mathrm{E}+03 & 9.426 \mathrm{E}-01 & 5.538 \mathrm{E}-01 & 9.426 \mathrm{E}-01 & 9.426 \mathrm{E}-01\end{array}$


*** INHALATION DOSE CALCULATION

USING DOSE CONVERSION FACTORS FROM ICRP-72 FOR MEMBERS OF THE PUBLIC

RESPIRABLE FRACTION $=1.000 \mathrm{E}+00$

BREATHING RATE $=3.330 \mathrm{E}-04(\mathrm{~m} \wedge 3 / \mathrm{s})$

RELEASE TIME FOR EXPONENTIAL DECAY FUNCTION $=1.000 \mathrm{E}+00$ ( $\mathrm{s}$ )

INTERNAL EXPOSURE TIME PERIOD $=5.000 \mathrm{E}+01$ (yr)

DEFAULT ELEMENT LUNG ABSORPTION TYPES RECOMMENDED BY ICRP-72

INHALATION DOSE CALCULATIONS FOR ADULT AGE

INHALATION EQUIVALENT DOSE ORDERED BY ORGAN (rem) FOR ADULT AGE

\begin{tabular}{|c|c|c|c|c|}
\hline INWIND & DIST & CES (m) & & \\
\hline GAN & & $E+02$ & +02 & 1.00 \\
\hline DRENALS & & $5 E-02$ & .89 & $.55 \mathrm{k}-2$ \\
\hline B_W & & 52 & & \\
\hline & & $E+01$ & $1.24 \mathrm{E}+00$ & $3.92 \mathrm{E}-01$ \\
\hline BRA & & 07 & 26 & $99 F-c$ \\
\hline BRE & & 2 & $?$ & 4 \\
\hline COLON & & & 2 & 8 \\
\hline ESOPHAGU & & -02 & $1.74 \mathrm{E}$ & $50 \mathrm{E}$ \\
\hline & & & & 41 \\
\hline KID & 9 & & 1. & 3 \\
\hline LIVER & 10 & 00 & $3.15 \mathrm{E}$ & 9. \\
\hline LLI_W, & 1 & 3. & 2. & 8.2 \\
\hline $\begin{array}{l}\text { LUNGS } \\
\text { MUSCLE }\end{array}$ & $\frac{12}{13}$ & $\begin{array}{l}1.79 \mathrm{E}+00 \\
2.80 \mathrm{~F}-02\end{array}$ & $\begin{array}{l}1.17 \mathrm{E}-01 \\
1.84 \mathrm{~F}-03\end{array}$ & $\begin{array}{l}3.71 \\
5.82\end{array}$ \\
\hline OVARIES & 14 & -02 & $2.33 \mathrm{E}$ & $7.39 \mathrm{E}$ \\
\hline PANCREAS & 15 & & $4.43 \mathrm{E}$ & 1.4 \\
\hline$R \_M$ & & & 1.0 & 3.1 \\
\hline $\begin{array}{l}\text { SI_I } \\
\text { SKT }\end{array}$ & 18 & $=-02$ & $\begin{array}{l}1.87 \mathrm{E}-03 \\
1.10 \mathrm{~F}-03\end{array}$ & 3 \\
\hline SPLEEN & & & $3.72 \mathrm{E}$ & \\
\hline & & & 10 & \\
\hline $\mathrm{TE}$ & & & & \\
\hline THYMU & 2 & & $4 \mathrm{E}$ & 5 \\
\hline & & & & \\
\hline & & & & \\
\hline JTERUS & & & $1.27 \mathrm{E}-03$ & 4.01 \\
\hline
\end{tabular}

INHALATION EQUIVALENT DOSE ORDERED BY DOSE (rem) FOR ADULT AGE DOWNWIND DISTANCES (m)

\begin{tabular}{lrccc} 
ORGAN & NO. & $1.00 \mathrm{E}+02$ & $5.00 \mathrm{E}+02$ & $1.00 \mathrm{E}+03$ \\
\hline BSURFACE & -1 & $1.89 \mathrm{E}+01$ & $1.24 \mathrm{E}+00$ & $3.92 \mathrm{E}-01$ \\
LIVER & 10 & $4.80 \mathrm{E}+00$ & $3.15 \mathrm{E}-01$ & $9.97 \mathrm{E}-02$ \\
LUNGS & 12 & $1.79 \mathrm{E}+00$ & $1.17 \mathrm{E}-01$ & $3.71 \mathrm{E}-02$ \\
R_MARROW & 16 & $1.54 \mathrm{E}+00$ & $1.01 \mathrm{E}-01$ & $3.19 \mathrm{E}-02$ \\
ET_AIR & 8 & $6.94 \mathrm{E}-01$ & $4.55 \mathrm{E}-02$ & $1.44 \mathrm{E}-02$ \\
KIDNEYS & 9 & $1.69 \mathrm{E}-01$ & $1.11 \mathrm{E}-02$ & $3.52 \mathrm{E}-03$ \\
ADRENALS & 1 & $7.45 \mathrm{E}-02$ & $4.89 \mathrm{E}-03$ & $1.55 \mathrm{E}-03$ \\
PANCREAS & 15 & $6.75 \mathrm{E}-02$ & $4.43 \mathrm{E}-03$ & $1.40 \mathrm{E}-03$ \\
SPLEEN & 19 & $5.67 \mathrm{E}-02$ & $3.72 \mathrm{E}-03$ & $1.18 \mathrm{E}-03$ \\
THYROID & 23 & $4.11 \mathrm{E}-02$ & $2.71 \mathrm{E}-03$ & $8.61 \mathrm{E}-04$ \\
ULI_WALL & 24 & $4.07 \mathrm{E}-02$ & $2.67 \mathrm{E}-03$ & $8.45 \mathrm{E}-04$ \\
LLI_WALL & 11 & $3.96 \mathrm{E}-02$ & $2.60 \mathrm{E}-03$ & $8.23 \mathrm{E}-04$ \\
COLON & 6 & $3.90 \mathrm{E}-02$ & $2.56 \mathrm{E}-03$ & $8.10 \mathrm{E}-04$ \\
OVARIES & 14 & $3.56 \mathrm{E}-02$ & $2.33 \mathrm{E}-03$ & $7.39 \mathrm{E}-04$ \\
ST_WALL & 20 & $2.99 \mathrm{E}-02$ & $1.97 \mathrm{E}-03$ & $6.27 \mathrm{E}-04$ \\
SI_WALL & 17 & $2.85 \mathrm{E}-02$ & $1.87 \mathrm{E}-03$ & $5.92 \mathrm{E}-04$ \\
MUSCLE & 13 & $2.80 \mathrm{E}-02$ & $1.84 \mathrm{E}-03$ & $5.82 \mathrm{E}-04$ \\
ESOPHAGU & 7 & $2.65 \mathrm{E}-02$ & $1.74 \mathrm{E}-03$ & $5.50 \mathrm{E}-04$ \\
THYMUS & 22 & $2.65 \mathrm{E}-02$ & $1.74 \mathrm{E}-03$ & $5.50 \mathrm{E}-04$ \\
TESTES & 21 & $2.57 \mathrm{E}-02$ & $1.69 \mathrm{E}-03$ & $5.34 \mathrm{E}-04$ \\
BREAST & 5 & $2.40 \mathrm{E}-02$ & $1.57 \mathrm{E}-03$ & $4.99 \mathrm{E}-04$ \\
UTERUS & 25 & $1.93 \mathrm{E}-02$ & $1.27 \mathrm{E}-03$ & $4.01 \mathrm{E}-04$ \\
BRAIN & 4 & $1.92 \mathrm{E}-02$ & $1.26 \mathrm{E}-03$ & $3.99 \mathrm{E}-04$ \\
SKIN & 18 & $1.68 \mathrm{E}-02$ & $1.10 \mathrm{E}-03$ & $3.50 \mathrm{E}-04$ \\
B_WALL & 2 & $1.59 \mathrm{E}-02$ & $1.04 \mathrm{E}-03$ & $3.30 \mathrm{E}-04$ \\
STIL & & & &
\end{tabular}




\begin{tabular}{|c|c|c|c|c|}
\hline $\begin{array}{l}\text { DOWNWIND } \\
\text { JRGAN }\end{array}$ & $\begin{array}{l}\text { DIST, } \\
\text { NO. }\end{array}$ & $\begin{array}{c}\text { NCES (m) } \\
1.00 \mathrm{~m}+02\end{array}$ & $5.00 \mathrm{E}+02$ & $1.00 \mathrm{E}+03$ \\
\hline $\begin{array}{l}\text { ADRENALS } \\
\text { B_WALL } \\
\text { BSURFACE } \\
\text { BRAIN } \\
\text { BREAST } \\
\text { COLON } \\
\text { ESOPHAGU } \\
\text { ET_AIR } \\
\text { KIDNEYS } \\
\text { LIVER } \\
\text { LLI_WALL } \\
\text { LUNGS } \\
\text { MUSCLE } \\
\text { OVARIES } \\
\text { PANCREAS } \\
\text { R_MARROW } \\
\text { SI_WALL } \\
\text { SKIN } \\
\text { SPLEEN } \\
\text { ST_WALL } \\
\text { TESTES } \\
\text { THYMUS } \\
\text { THYROID } \\
\text { ULI_WALL } \\
\text { UTERUS } \\
\text { E_50 }\end{array}$ & $\begin{array}{r}3 \\
4 \\
5 \\
6 \\
7 \\
8 \\
9 \\
10 \\
11 \\
12 \\
13 \\
14 \\
15 \\
16 \\
17 \\
18 \\
19 \\
20 \\
21 \\
22 \\
23 \\
24 \\
25 \\
26\end{array}$ & $\begin{array}{l}3.73 \mathrm{E}-03 \\
7.94 \mathrm{E}-04 \\
1.89 \mathrm{E}-01 \\
9.60 \mathrm{E}-04 \\
1.20 \mathrm{E}-03 \\
4.68 \mathrm{E}-03 \\
1.32 \mathrm{E}-03 \\
3.47 \mathrm{E}-02 \\
8.47 \mathrm{E}-03 \\
2.40 \mathrm{E}-01 \\
1.98 \mathrm{E}-03 \\
2.15 \mathrm{E}-01 \\
1.40 \mathrm{E}-03 \\
1.78 \mathrm{E}-03 \\
3.38 \mathrm{E}-03 \\
1.84 \mathrm{E}-01 \\
1.42 \mathrm{E}-03 \\
1.68 \mathrm{E}-04 \\
2.83 \mathrm{E}-03 \\
5.98 \mathrm{E}-03 \\
1.29 \mathrm{E}-03 \\
1.32 \mathrm{E}-03 \\
2.05 \mathrm{E}-03 \\
2.04 \mathrm{E}-03 \\
9.66 \mathrm{E}-04 \\
8.51 \mathrm{E}-01\end{array}$ & $\begin{array}{l}2.45 \mathrm{E}-04 \\
5.21 \mathrm{E}-05 \\
1.24 \mathrm{E}-02 \\
6.30 \mathrm{E}-05 \\
7.87 \mathrm{E}-05 \\
3.07 \mathrm{E}-04 \\
8.69 \mathrm{E}-05 \\
2.27 \mathrm{E}-03 \\
5.56 \mathrm{E}-04 \\
1.57 \mathrm{E}-02 \\
1.30 \mathrm{E}-04 \\
1.41 \mathrm{E}-02 \\
9.19 \mathrm{E}-05 \\
1.17 \mathrm{E}-04 \\
2.21 \mathrm{E}-04 \\
1.21 \mathrm{E}-02 \\
9.35 \mathrm{E}-05 \\
1.10 \mathrm{E}-05 \\
1.86 \mathrm{E}-04 \\
3.95 \mathrm{E}-04 \\
8.43 \mathrm{E}-05 \\
8.69 \mathrm{E}-05 \\
1.35 \mathrm{E}-04 \\
1.33 \mathrm{E}-04 \\
6.33 \mathrm{E}-05 \\
5.58 \mathrm{E}-02\end{array}$ & $\begin{array}{l}7.74 \mathrm{E}-05 \\
1.65 \mathrm{E}-05 \\
3.92 \mathrm{E}-03 \\
2.00 \mathrm{E}-05 \\
2.49 \mathrm{E}-05 \\
9.72 \mathrm{E}-05 \\
2.75 \mathrm{E}-05 \\
7.19 \mathrm{E}-04 \\
1.76 \mathrm{E}-04 \\
4.98 \mathrm{E}-03 \\
4.11 \mathrm{E}-05 \\
4.45 \mathrm{E}-03 \\
2.91 \mathrm{E}-05 \\
3.70 \mathrm{E}-05 \\
7.01 \mathrm{E}-05 \\
3.83 \mathrm{E}-03 \\
2.96 \mathrm{E}-05 \\
3.50 \mathrm{E}-06 \\
5.89 \mathrm{E}-05 \\
1.25 \mathrm{E}-04 \\
2.67 \mathrm{E}-05 \\
2.75 \mathrm{E}-05 \\
4.31 \mathrm{E}-05 \\
4.23 \mathrm{E}-05 \\
2.01 \mathrm{E}-05 \\
1.77 \mathrm{E}-02\end{array}$ \\
\hline
\end{tabular}

*** INGESTION DOSE CALCULATION

USING DOSE CONVERSION FACTORS FROM ICRP-72 FOR MEMBERS OF THE PUBLIC RELEASE TIME FOR EXPONENTIAL DECAY FUNCTION $=1.000 \mathrm{E}+00(\mathrm{~s})$

INTERNAL EXPOSURE TIME PERIOD $=5.000 E+01(\mathrm{yr})$

INGESTION CALCULATIONS MADE USING CODE CALCULATED CONSTANTS

INGESTION CONSTANTS:

5.20E+02 STORED VEGETABLE USAGE FACTOR (KG/YR)

6.40E+01 FRESH VEGETABLE USAGE FACTOR (KG/YR)

1.10E+02 MEAT USAGE FACTOR (KG/YR)

3.10E+02 MILK USAGE FACTOR (L/YR)

7.60E-01 FRACTION OF STORED VEGETABLES FROM GARDEN

1.00E+00 FRACTION OF FRESH VEGETABLES FROM GARDEN

5.70E-01 RETENTION FACTOR FOR ACTIVITY ON FORAGE

2.00E-01 RETENTION FACTOR FOR ACTIVITY ON VEGETABLES

1.00E+00 RETENTION FACTOR FOR IODINES

2.10E-03 REMOVAL RATE CONSTANT FOR CROPS $(1 / \mathrm{H})$

6.00E+01 VEGETABLE EXPOSURE TIME TO PLUME FOR CHRONIC RELEASE (D)

3.00E+01 FORAGE EXPOSURE TIME TO PLUME FOR CHRONIC RELEASE (D)

1.00E+00 HTO REMOVAL HALF TIME (D)

2.25E+02 EFFECTIVE SURFACE SOIL DENSITY (KG/SQ M)

6.00E+01 STORED VEGETABLE HOLDUP TIME AFTER HARVEST (D)

1.00E+00 FRESH VEGETABLE HOLDUP TIME AFTER HARVEST (D)

1.60E+01 ANIMALS DAILY FORAGE FEED (KG/D)

2.00E+00 FEED-MILK-RECEPTOR TRANSFER TIME (D)

2.00E+01 SLAUGHTER TO CONSUMPTION TIME (D)

4.00E-01 FRACTION OF YEAR ON PASTURE

4.30E-01 PASTURE FEED FRACTION

9.00E+01 STORED FEED STORAGE TIME

2.00E+00 VEGETABLE VEGETATION YIELD (KG/SQ M)

2.80E-01 FORAGE VEGETATION YIELD (KG/SQ M)

4.90E+00 ABSOLUTE HUMIDITY (G/CU M)

CHRONIC RELEASE - ANNUAL DOSE

ACTIVITY BUILDUP IN SOIL OVER $1.500 \mathrm{E}+01$ (YR) 
ICRP-72 INGESTION DOSE CALCULATIONS FOR ADULT AGE INGESTION EQUIVALENT DOSE ORDERED BY ORGAN (rem) FOR ADULT AGE

$\begin{array}{lrlll}\text { DOWNWIND } & \text { DISTANCES }(\mathrm{m}) & & \\ \text { ORGAN } & \text { NO. } & 1.00 \mathrm{E}+02 & 5.00 \mathrm{E}+02 & 1.00 \mathrm{E}+03 \\ ------ & --- & ------ & ---1--- & ------ \\ \text { ADRENALS } & 1 & 6.77 \mathrm{E}-01 & 4.37 \mathrm{E}-02 & 1.36 \mathrm{E}-02 \\ \text { B_WALL } & 2 & 1.35 \mathrm{E}+00 & 8.64 \mathrm{E}-02 & 2.68 \mathrm{E}-02 \\ \text { BSURFACE } & 3 & 4.38 \mathrm{E}+00 & 2.87 \mathrm{E}-01 & 9.06 \mathrm{E}-02 \\ \text { BRAIN } & 4 & 9.09 \mathrm{E}-01 & 5.89 \mathrm{E}-02 & 1.85 \mathrm{E}-02 \\ \text { BREAST } & 5 & 6.05 \mathrm{E}-01 & 3.89 \mathrm{E}-02 & 1.21 \mathrm{E}-02 \\ \text { COLON } & 6 & 1.02 \mathrm{E}+00 & 6.61 \mathrm{E}-02 & 2.07 \mathrm{E}-02 \\ \text { ESOPHAGU } & 7 & 6.26 \mathrm{E}-01 & 4.03 \mathrm{E}-02 & 1.26 \mathrm{E}-02 \\ \text { ET_AIR } & 8 & 6.37 \mathrm{E}-01 & 4.10 \mathrm{E}-02 & 1.28 \mathrm{E}-02 \\ \text { KIDNEYS } & 9 & 1.87 \mathrm{E}+00 & 1.22 \mathrm{E}-01 & 3.83 \mathrm{E}-02 \\ \text { LIVER } & 10 & 1.24 \mathrm{E}+00 & 8.05 \mathrm{E}-02 & 2.53 \mathrm{E}-02 \\ \text { LLI_WALL } & 11 & 1.26 \mathrm{E}+00 & 8.15 \mathrm{E}-02 & 2.55 \mathrm{E}-02 \\ \text { LUNGS } & 12 & 6.29 \mathrm{E}-01 & 4.05 \mathrm{E}-02 & 1.26 \mathrm{E}-02 \\ \text { MUSCLE } & 13 & 6.29 \mathrm{E}-01 & 4.05 \mathrm{E}-02 & 1.26 \mathrm{E}-02 \\ \text { OVARIES } & 14 & 6.66 \mathrm{E}-01 & 4.30 \mathrm{E}-02 & 1.34 \mathrm{E}-02 \\ \text { PANCREAS } & 15 & 6.75 \mathrm{E}-01 & 4.36 \mathrm{E}-02 & 1.36 \mathrm{E}-02 \\ \text { R_MARROW } & 16 & 1.15 \mathrm{E}+00 & 7.46 \mathrm{E}-02 & 2.34 \mathrm{E}-02 \\ \text { SI_WALL } & 17 & 6.84 \mathrm{E}-01 & 4.41 \mathrm{E}-02 & 1.38 \mathrm{E}-02 \\ \text { SKIN } & 18 & 6.03 \mathrm{E}-01 & 3.88 \mathrm{E}-02 & 1.21 \mathrm{E}-02 \\ \text { SPLEEN } & 19 & 1.26 \mathrm{E}+00 & 8.19 \mathrm{E}-02 & 2.57 \mathrm{E}-02 \\ \text { ST_WALL } & 20 & 8.52 \mathrm{E}-01 & 5.49 \mathrm{E}-02 & 1.71 \mathrm{E}-02 \\ \text { TESTES } & 21 & 6.16 \mathrm{E}-01 & 3.97 \mathrm{E}-02 & 1.24 \mathrm{E}-02 \\ \text { THYMUS } & 22 & 6.26 \mathrm{E}-01 & 4.03 \mathrm{E}-02 & 1.26 \mathrm{E}-02 \\ \text { THYROID } & 23 & 1.49 \mathrm{E}+01 & 9.78 \mathrm{E}-01 & 3.09 \mathrm{E}-01 \\ \text { ULI_WALL } & 24 & 8.49 \mathrm{E}-01 & 5.48 \mathrm{E}-02 & 1.72 \mathrm{E}-02 \\ \text { UTERUS } & 25 & 6.51 \mathrm{E}-01 & 4.20 \mathrm{E}-02 & 1.31 \mathrm{E}-02 \\ \text { UTER } & & & & \end{array}$

INGESTION EQUIVALENT DOSE ORDERED BY DOSE (rem) FOR ADULT AGE DOWNWIND DISTANCES (m)

$\begin{array}{lrccc}\text { ORGAN } & \text { NO. } & 1.00 \mathrm{E}+02 & 5.00 \mathrm{E}+02 & 1.00 \mathrm{E}+03 \\ ------ & -- & ------ & ---1-- & ------1 \\ \text { THYROID } & 23 & 1.49 \mathrm{E}+01 & 9.78 \mathrm{E}-01 & 3.09 \mathrm{E}-01 \\ \text { BSURFACE } & 3 & 4.38 \mathrm{E}+00 & 2.87 \mathrm{E}-01 & 9.06 \mathrm{E}-02 \\ \text { KIDNEYS } & 9 & 1.87 \mathrm{E}+00 & 1.22 \mathrm{E}-01 & 3.83 \mathrm{E}-02 \\ \text { B_WALL } & 2 & 1.35 \mathrm{E}+00 & 8.64 \mathrm{E}-02 & 2.68 \mathrm{E}-02 \\ \text { SPLEEN } & 19 & 1.26 \mathrm{E}+00 & 8.19 \mathrm{E}-02 & 2.57 \mathrm{E}-02 \\ \text { LLI_WALL } & 11 & 1.26 \mathrm{E}+00 & 8.15 \mathrm{E}-02 & 2.55 \mathrm{E}-02 \\ \text { LIVER } & 10 & 1.24 \mathrm{E}+00 & 8.05 \mathrm{E}-02 & 2.53 \mathrm{E}-02 \\ \text { R_MARROW } & 16 & 1.15 \mathrm{E}+00 & 7.46 \mathrm{E}-02 & 2.34 \mathrm{E}-02 \\ \text { COLON } & 6 & 1.02 \mathrm{E}+00 & 6.61 \mathrm{E}-02 & 2.07 \mathrm{E}-02 \\ \text { BRAIN } & 4 & 9.09 \mathrm{E}-01 & 5.89 \mathrm{E}-02 & 1.85 \mathrm{E}-02 \\ \text { ST_WALL } & 20 & 8.52 \mathrm{E}-01 & 5.49 \mathrm{E}-02 & 1.71 \mathrm{E}-02 \\ \text { ULI_WALL } & 24 & 8.49 \mathrm{E}-01 & 5.48 \mathrm{E}-02 & 1.72 \mathrm{E}-02 \\ \text { SI_WALL } & 17 & 6.84 \mathrm{E}-01 & 4.41 \mathrm{E}-02 & 1.38 \mathrm{E}-02 \\ \text { ADRENALS } & 1 & 6.77 \mathrm{E}-01 & 4.37 \mathrm{E}-02 & 1.36 \mathrm{E}-02 \\ \text { PANCREAS } & 15 & 6.75 \mathrm{E}-01 & 4.36 \mathrm{E}-02 & 1.36 \mathrm{E}-02 \\ \text { OVARIES } & 14 & 6.66 \mathrm{E}-01 & 4.30 \mathrm{E}-02 & 1.34 \mathrm{E}-02 \\ \text { UTERUS } & 25 & 6.51 \mathrm{E}-01 & 4.20 \mathrm{E}-02 & 1.31 \mathrm{E}-02 \\ \text { ET_AIR } & 8 & 6.37 \mathrm{E}-01 & 4.10 \mathrm{E}-02 & 1.28 \mathrm{E}-02 \\ \text { MUSCLE } & 13 & 6.29 \mathrm{E}-01 & 4.05 \mathrm{E}-02 & 1.26 \mathrm{E}-02 \\ \text { LUNGS } & 12 & 6.29 \mathrm{E}-01 & 4.05 \mathrm{E}-02 & 1.26 \mathrm{E}-02 \\ \text { ESOPHAGU } & 7 & 6.26 \mathrm{E}-01 & 4.03 \mathrm{E}-02 & 1.26 \mathrm{E}-02 \\ \text { THYMUS } & 22 & 6.26 \mathrm{E}-01 & 4.03 \mathrm{E}-02 & 1.26 \mathrm{E}-02 \\ \text { TESTES } & 21 & 6.16 \mathrm{E}-01 & 3.97 \mathrm{E}-02 & 1.24 \mathrm{E}-02 \\ \text { BREAST } & 5 & 6.05 \mathrm{E}-01 & 3.89 \mathrm{E}-02 & 1.21 \mathrm{E}-02 \\ \text { SKIN } & 18 & 6.03 \mathrm{E}-01 & 3.88 \mathrm{E}-02 & 1.21 \mathrm{E}-02\end{array}$




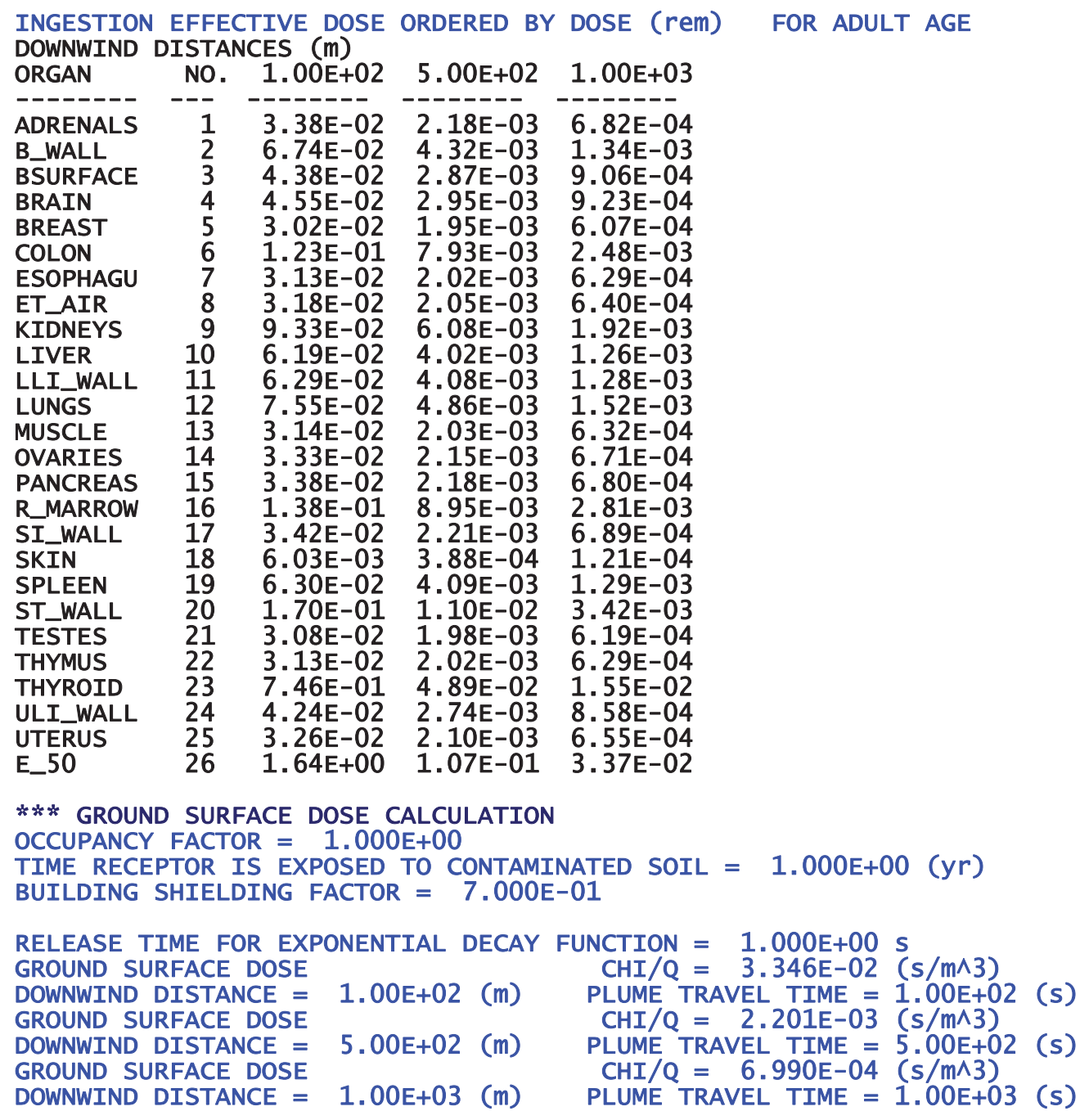




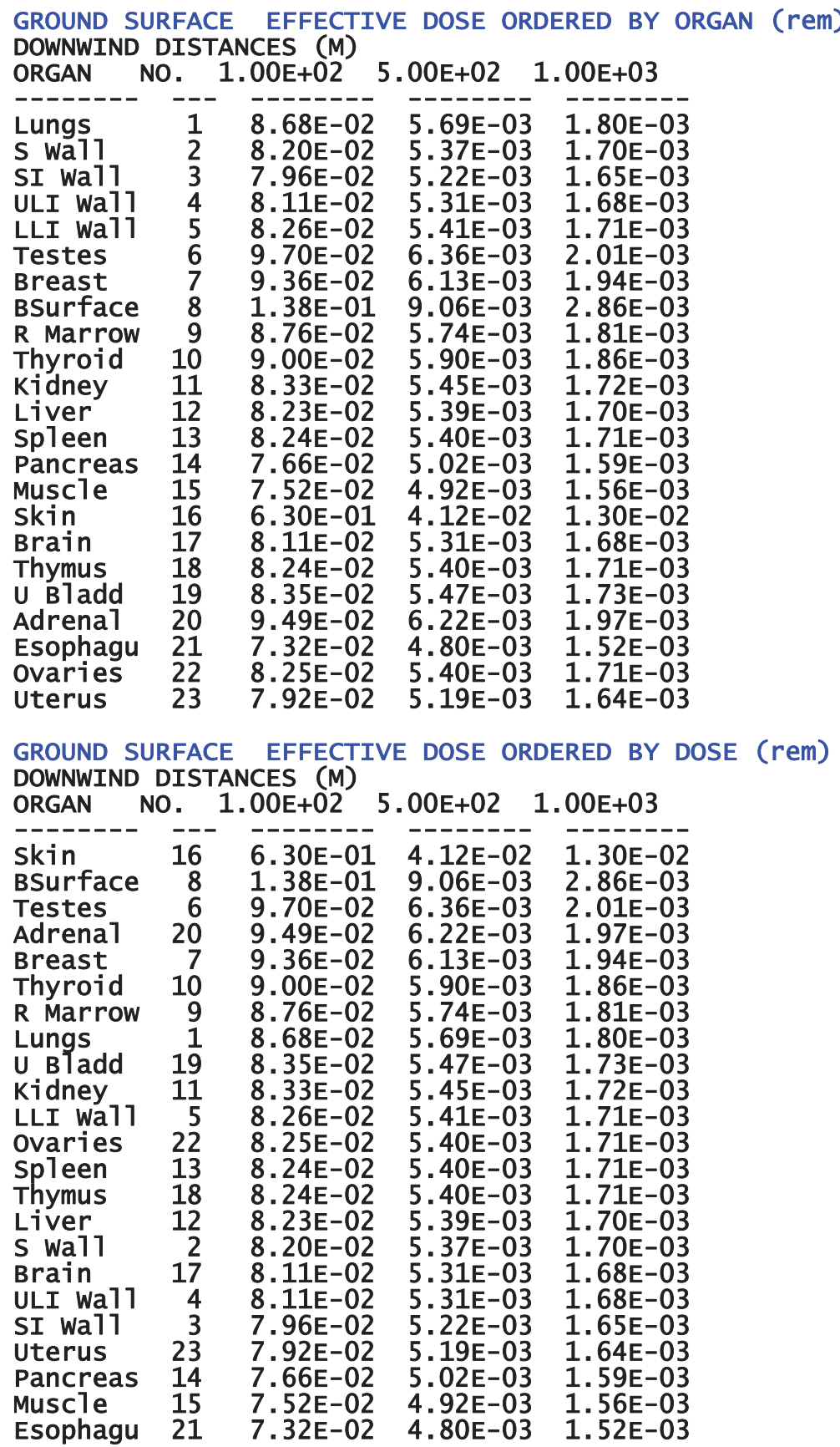




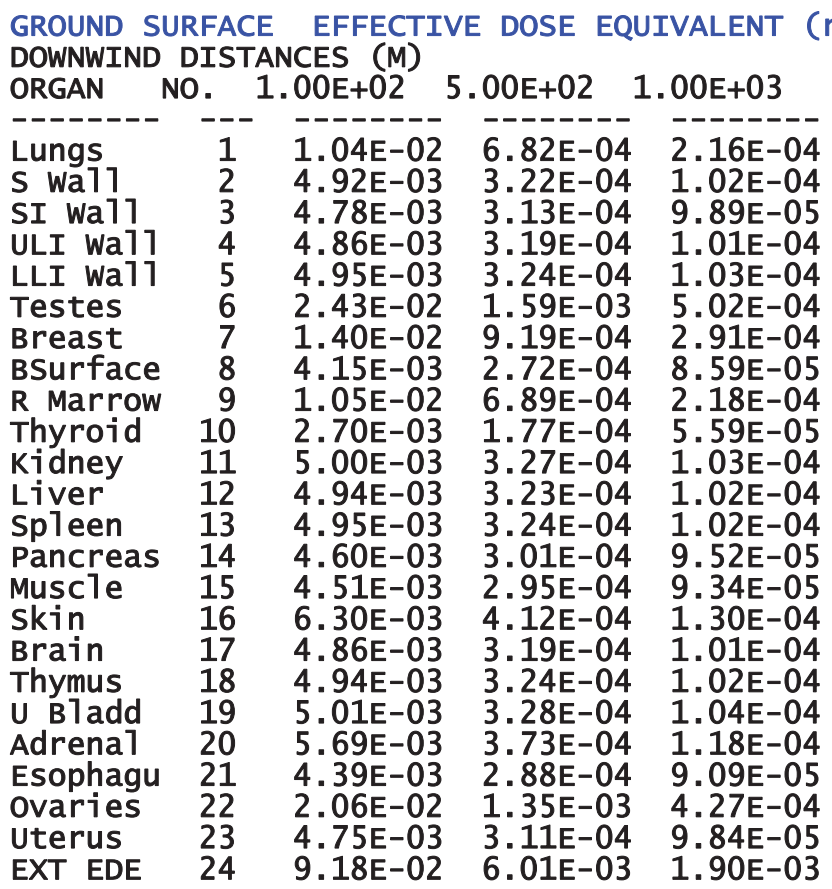

*** DOSE SUMMARY

ICRP-72 INHALATION DOSE CALCULATIONS MADE WITH ADULT INTAKE AGE ICRP-72 INGESTION DOSE CALCULATIONS MADE WITH ADULT INTAKE AGE SUM OF CONTRIBUTIONS TO THE EFFECTIVE DOSE (rem) DOWNWIND DISTANCE $=1.00 \mathrm{E}+02(\mathrm{~m})$

SORTED BY PERCENT OF EFFECTIVE DOSE

NUCLIDE

$531290 \quad I-129$

$170360 \mathrm{C} 1-36$

$621460 \mathrm{Sm}-146$

$641480 \mathrm{Gd}-148$

$822100 \mathrm{~Pb}-210$

$621470 \mathrm{Sm}-147$

$641520 \mathrm{Gd}-152$

$10030 \mathrm{H}-3$

$801940 \mathrm{Hg}-194$

531250 I-125

571370 La-137

832101 Bi-210m

$942390 \mathrm{Pu}-239$

$260600 \mathrm{Fe}-60$

531260 I-126

551370 Cs -137

842100 Po-210

531310 I-131

$380900 \mathrm{Sr}-90$

761940 os -194

491150 In-115

$481130 \mathrm{~cd}-113$

430980 Tc- 98

$320680 \mathrm{Ge}-68$

551340 Cs -134

481131 Cd-113m

$190400 \mathrm{~K}-40$

$220440 \mathrm{Ti}-44$

$410940 \mathrm{Nb}-94$

531240 I-124

501260 sn-126

791940 Au-194

$110220 \mathrm{Na}-22$ INTERNAL EXTERNAL

$\begin{array}{lc}------ & ---- \\ 6.44 \mathrm{E}-01 & - \\ 4.92 \mathrm{E}-01 & - \\ 1.69 \mathrm{E}-01 & - \\ 1.68 \mathrm{E}-01 & - \\ 1.61 \mathrm{E}-01 & - \\ 1.48 \mathrm{E}-01 & - \\ 1.23 \mathrm{E}-01 & - \\ 8.70 \mathrm{E}-02 & - \\ 6.42 \mathrm{E}-02 & - \\ 6.38 \mathrm{E}-02 & - \\ 5.28 \mathrm{E}-02 & - \\ 5.16 \mathrm{E}-02 & - \\ 3.21 \mathrm{E}-02 & - \\ 3.09 \mathrm{E}-02 & - \\ 2.08 \mathrm{E}-02 & - \\ 1.37 \mathrm{E}-02 & - \\ 1.36 \mathrm{E}-02 & - \\ 1.11 \mathrm{E}-02 & - \\ 8.81 \mathrm{E}-03 & - \\ 7.95 \mathrm{E}-03 & - \\ 7.47 \mathrm{E}-03 & - \\ 7.33 \mathrm{E}-03 & - \\ 6.68 \mathrm{E}-03 & - \\ 6.49 \mathrm{E}-03 & - \\ 6.09 \mathrm{E}-03 & - \\ 6.05 \mathrm{E}-03 & - \\ 5.60 \mathrm{E}-03 & - \\ 4.63 \mathrm{E}-03 & - \\ 4.43 \mathrm{E}-03 & - \\ 3.62 \mathrm{E}-03 & - \\ 3.58 \mathrm{E}-03 & - \\ 3.50 \mathrm{E}-03 & - \\ 3.47 \mathrm{E}-03 & - \\ & -\end{array}$

TOTAL

\begin{tabular}{lr}
\hline $6.44 \mathrm{E}-01$ & $2.58 \mathrm{E}+01$ \\
$4.92 \mathrm{E}-01$ & $1.97 \mathrm{E}+01$ \\
$1.69 \mathrm{E}-01$ & $6.76 \mathrm{E}+00$ \\
$1.68 \mathrm{E}-01$ & $6.75 \mathrm{E}+00$ \\
$1.61 \mathrm{E}-01$ & $6.46 \mathrm{E}+00$ \\
$1.48 \mathrm{E}-01$ & $5.91 \mathrm{E}+00$ \\
$1.23 \mathrm{E}-01$ & $4.91 \mathrm{E}+00$ \\
$8.70 \mathrm{E}-02$ & $3.48 \mathrm{E}+00$ \\
$6.42 \mathrm{E}-02$ & $2.57 \mathrm{E}+00$ \\
$6.38 \mathrm{E}-02$ & $2.56 \mathrm{E}+00$ \\
$5.28 \mathrm{E}-02$ & $2.11 \mathrm{E}+00$ \\
$5.16 \mathrm{E}-02$ & $2.07 \mathrm{E}+00$ \\
$3.21 \mathrm{E}-02$ & $1.29 \mathrm{E}+00$ \\
$3.09 \mathrm{E}-02$ & $1.24 \mathrm{E}+00$ \\
$2.08 \mathrm{E}-02$ & $8.33 \mathrm{E}-01$ \\
$1.37 \mathrm{E}-02$ & $5.49 \mathrm{E}-01$ \\
$1.36 \mathrm{E}-02$ & $5.43 \mathrm{E}-01$ \\
$1.11 \mathrm{E}-02$ & $4.43 \mathrm{E}-01$ \\
$8.81 \mathrm{E}-03$ & $3.53 \mathrm{E}-01$ \\
$7.95 \mathrm{E}-03$ & $3.18 \mathrm{E}-01$ \\
$7.47 \mathrm{E}-03$ & $2.99 \mathrm{E}-01$ \\
$7.33 \mathrm{E}-03$ & $2.94 \mathrm{E}-01$ \\
$6.68 \mathrm{E}-03$ & $2.68 \mathrm{E}-01$ \\
$6.49 \mathrm{E}-03$ & $2.60 \mathrm{E}-01$ \\
$6.09 \mathrm{E}-03$ & $2.44 \mathrm{E}-01$ \\
$6.05 \mathrm{E}-03$ & $2.42 \mathrm{E}-01$ \\
$5.60 \mathrm{E}-03$ & $2.24 \mathrm{E}-01$ \\
$4.63 \mathrm{E}-03$ & $1.85 \mathrm{E}-01$ \\
$4.43 \mathrm{E}-03$ & $1.78 \mathrm{E}-01$ \\
$3.62 \mathrm{E}-03$ & $1.45 \mathrm{E}-01$ \\
$3.58 \mathrm{E}-03$ & $1.43 \mathrm{E}-01$ \\
$3.50 \mathrm{E}-03$ & $1.40 \mathrm{E}-01$ \\
$3.47 \mathrm{E}-03$ & $1.39 \mathrm{E}-01$
\end{tabular}


SORTED BY PERCENT OF EFFECTIVE DOSE

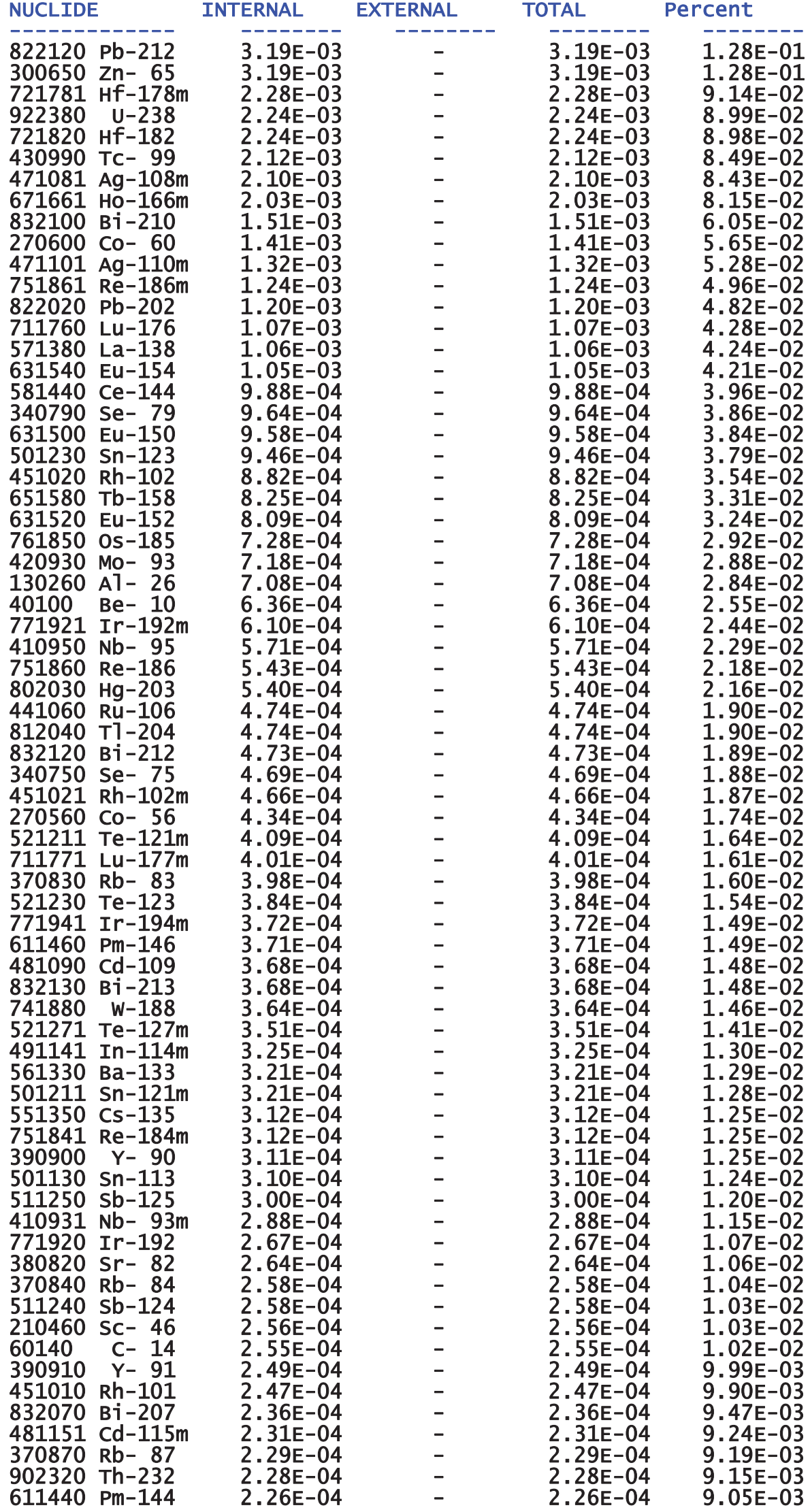


SORTED BY PERCENT OF EFFECTIVE DOSE

\begin{tabular}{|c|c|c|c|c|c|}
\hline NUCLIDE & & INTERNAL & EXTERNAL & TOTAL & Percent \\
\hline & & 223501 & & 27250 & בר \\
\hline $\begin{array}{l}521231 \\
200450\end{array}$ & Te-123m & $2.23 \mathrm{E}-04$ & - & 2. 23E-04 & 8.93E-03 \\
\hline 691700 & $\mathrm{Tm}-170$ & $\begin{array}{l}2.21 \mathrm{E}-04 \\
2.20 \mathrm{E}-04\end{array}$ & - & $\begin{array}{l}2.2 \mathrm{LE}-\mathrm{U} \\
2.20 \mathrm{~F}-04\end{array}$ & $8.88 \mathrm{E}-03$ \\
\hline 43097 & TC- 97 & $2.17 \mathrm{E}-04$ & _ & $2.17 \mathrm{E}-04$ & 0 \\
\hline & $T b-160$ & $2.11 \mathrm{E}-04$ & _- & $2.11 \mathrm{E}-04$ & $E-03$ \\
\hline & sn-119m & $2.10 \mathrm{E}-04$ & - & $2.10 \mathrm{E}-04$ & $1 E-03$ \\
\hline 260 & $\mathrm{sb}-126$ & $2.06 \mathrm{E}-04$ & - & $2.06 \mathrm{E}-04$ & $E-0$ \\
\hline 400 & $Z r-93$ & $2.05 \mathrm{E}-04$ & _ & $2.05 \mathrm{E}-04$ & $20 \mathrm{E}-03$ \\
\hline & $\mathrm{Ca}-41$ & $1.97 \mathrm{E}-04$ & - & $1.97 \mathrm{E}-04$ & $E-03$ \\
\hline 291 & $\mathrm{Te}-129 \mathrm{~m}$ & $1.94 \mathrm{E}-04$ & - & $1.94 \mathrm{E}-04$ & $7.77 \mathrm{E}-03$ \\
\hline 380890 & $S r-89$ & 1.92E-04 & - & $1.92 \mathrm{E}-04$ & $7.68 \mathrm{E}-03$ \\
\hline 731820 & Ta-182 & $1.88 \mathrm{E}-04$ & - & $1.88 \mathrm{E}-04$ & $7.53 \mathrm{E}-03$ \\
\hline & $P-32$ & $1.86 \mathrm{E}-04$ & - & $1.86 \mathrm{E}-04$ & $E-03$ \\
\hline & Tc- $97 \mathrm{~m}$ & $1.85 \mathrm{E}-04$ & - & $1.85 \mathrm{E}-04$ & $E-0$ \\
\hline 390880 & $Y-88$ & $1.82 \mathrm{E}-04$ & - & $1.82 \mathrm{E}-04$ & $7.28 \mathrm{E}-03$ \\
\hline 822140 & $\mathrm{~Pb}-214$ & $1.63 \mathrm{E}-04$ & - & $1.63 \mathrm{E}-04$ & $6.51 \mathrm{E}-03$ \\
\hline 590 & $\mathrm{Fe}-59$ & $1.61 \mathrm{E}-04$ & - & $1.61 \mathrm{E}-04$ & $6.46 \mathrm{E}-03$ \\
\hline & $\mathrm{Re}-184$ & $1.61 \mathrm{E}-04$ & - & $1.61 \mathrm{E}-04$ & $6.44 \mathrm{E}-03$ \\
\hline 81 & $\mathrm{Pm}-148 \mathrm{~m}$ & $1.58 \mathrm{E}-04$ & - & $1.58 \mathrm{E}-04$ & $6.32 \mathrm{E}-0$ \\
\hline 400950 & $\mathrm{Zr}-95$ & $1.48 \mathrm{E}-04$ & - & $1.48 \mathrm{E}-04$ & $5.93 \mathrm{E}-03$ \\
\hline 771940 & Ir-194 & $1.46 \mathrm{E}-04$ & - & $1.46 \mathrm{E}-04$ & $5.86 \mathrm{E}-0$ \\
\hline 50 & Eu-155 & $1.44 \mathrm{E}-04$ & - & $1.44 \mathrm{E}-04$ & 5.7 \\
\hline & $\mathrm{Pb}-211$ & $1.41 \mathrm{E}-04$ & - & $1.41 \mathrm{E}-04$ & -0 \\
\hline 370860 & $R b-86$ & $1.39 \mathrm{E}-04$ & - & $1.39 \mathrm{E}-04$ & $5.58 \mathrm{E}-03$ \\
\hline 832140 & Вi-214 & 1. 37E-04 & - & 1.37E-04 & $5.47 \mathrm{E}-03$ \\
\hline 270580 & Co- 58 & $1.22 \mathrm{E}-04$ & - & $1.22 \mathrm{E}-04$ & $4.89 \mathrm{E}-0$ \\
\hline 50 & Cs-136 & $1.18 \mathrm{E}-04$ & - & $1.18 \mathrm{E}-04$ & $4.74 \mathrm{E}-0$ \\
\hline & $\mathrm{Hf}-181$ & $1.16 \mathrm{E}-04$ & _ & $1.16 \mathrm{E}-04$ & 4.6 \\
\hline 761910 & Os-191 & $1.15 \mathrm{E}-04$ & - & $1.15 \mathrm{E}-04$ & $4.59 \mathrm{E}-03$ \\
\hline 711741 & Lu-174m & 1.11E-04 & - & $1.11 \mathrm{E}-04$ & $4.46 \mathrm{E}-03$ \\
\hline 480 & Eu-148 & $1.11 \mathrm{E}-04$ & - & $1.11 \mathrm{E}-04$ & $4.45 E-03$ \\
\hline 400 & $\mathrm{Ba}-140$ & $1.11 \mathrm{E}-04$ & - & $1.11 \mathrm{E}-04$ & $4.43 E-C$ \\
\hline 70 & $\mathrm{Pm}-147$ & $1.06 \mathrm{E}-04$ & - & $1.06 \mathrm{E}-04$ & $4.25 \mathrm{E}-0$ \\
\hline 250 & $\mathrm{sn}-125$ & $1.05 \mathrm{E}-04$ & - & $1.05 \mathrm{E}-04$ & $4.20 \mathrm{E}-0$ \\
\hline 812020 & T1-202 & $1.04 \mathrm{E}-04$ & - & $1.04 \mathrm{E}-04$ & $4.16 \mathrm{E}-03$ \\
\hline 380 & Cs-138 & $1.02 \mathrm{E}-04$ & - & $1.02 \mathrm{E}-04$ & $4.11 \mathrm{E}-0$ \\
\hline & Lu-174 & $9.76 \mathrm{E}-05$ & - & 9.76 & $3.91 \mathrm{E}-0$ \\
\hline 540 & $M n-54$ & 9.27 & - & 05 & $71 \mathrm{E}-\mathrm{c}$ \\
\hline 521251 & $\mathrm{Te}-125 \mathrm{~m}$ & $9.20 \mathrm{E}$ & - & $9.20 \mathrm{E}-05$ & $3.69 \mathrm{E}-0$ \\
\hline 611480 & Pm-148 & $8.95 \mathrm{E}$ & - & 8.95 & $3.59 \mathrm{E}-0$ \\
\hline 880 & $\mathrm{Re}-188$ & $8.74 \mathrm{E}$ & _ & 8.74 & $3.50 \mathrm{E}$ \\
\hline & $\mathrm{Zr}-88$ & 8.73 & - & 8.7 & $3.50 \mathrm{E}-\mathrm{C}$ \\
\hline & $\mathrm{Fe}-55$ & 8.66 & - & 5 & $47 \mathrm{E}$ \\
\hline 320 & Te-132 & 8.42 & - & 8.42 & $3.37 \mathrm{E}-0$ \\
\hline 370880 & $R b-88$ & 8.37 & - & 8.37 & $3.36 \mathrm{E}-0$ \\
\hline 721791 & Hf- $179 m$ & 8.17 & - & 8.1 & 3.2 \\
\hline 741850 & W-185 & 8 & - & 8. & 3 \\
\hline 160350 & $s-3$ & 8 & 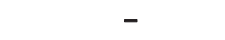 & 8. & $20 \mathrm{E}-\mathrm{C}$ \\
\hline 631560 & $\mathrm{Eu}-156$ & 7.72 & - & 7. & $09 F_{-}$ \\
\hline 510 & Sm-151 & $7.24 \mathrm{E}-$ & - & 7.24 & $2.90 \mathrm{E}-0$ \\
\hline 410 & $\mathrm{Ce}-141$ & 6. & - & 6. & 2 \\
\hline & $\mathrm{Yb}-169$ & 6 & - & 6 & 2 \\
\hline & $\mathrm{Ag}-106 \mathrm{~m}$ & 6 & - & 5 & $56 E-c$ \\
\hline & $\mathrm{Ag}-11$ & & - & & 2 \\
\hline 570 & $\mathrm{CO}-5$ & 6 & 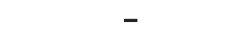 & 6 & 2 \\
\hline & $\mathrm{Sn}-117 \mathrm{~m}$ & 6. & - & & 2 \\
\hline & $V-48$ & & _ & & 41 \\
\hline 531330 & I-133 & & - & & $8 \mathrm{E}-0$ \\
\hline & $\mathrm{Gd}-153$ & & - & & $36 \mathrm{E}-0$ \\
\hline & Au-195 & & - & & \\
\hline & Te-121 & 5 & - & & $10 \mathrm{r}$ \\
\hline 30 & $P-3 \overline{3}$ & & - & 4.95 & $99 \mathrm{E}-$ \\
\hline & As- 74 & & - & 4.94 & $1.98 \mathrm{E}-0$ \\
\hline & $\mathrm{Pm}-145$ & & - & 4. & $1.96 \mathrm{E}-$ \\
\hline & $\mathrm{Ni}-63$ & & - & & \\
\hline & $\mathrm{Ca}-47$ & & - & 4. & $1.93 \mathrm{E}$ \\
\hline & Lu-177 & & - & 4.78 & $91 \mathrm{E}-\mathrm{c}$ \\
\hline & $\mathrm{Pr}-143$ & & - & $4.68 \mathrm{E}$ & $1.87 t-4$ \\
\hline & $\mathrm{Pm}-1$ & J & - & $6 E-0$ & $37 \mathrm{E}-$ \\
\hline
\end{tabular}


SORTED BY PERCENT OF EFFECTIVE DOSE

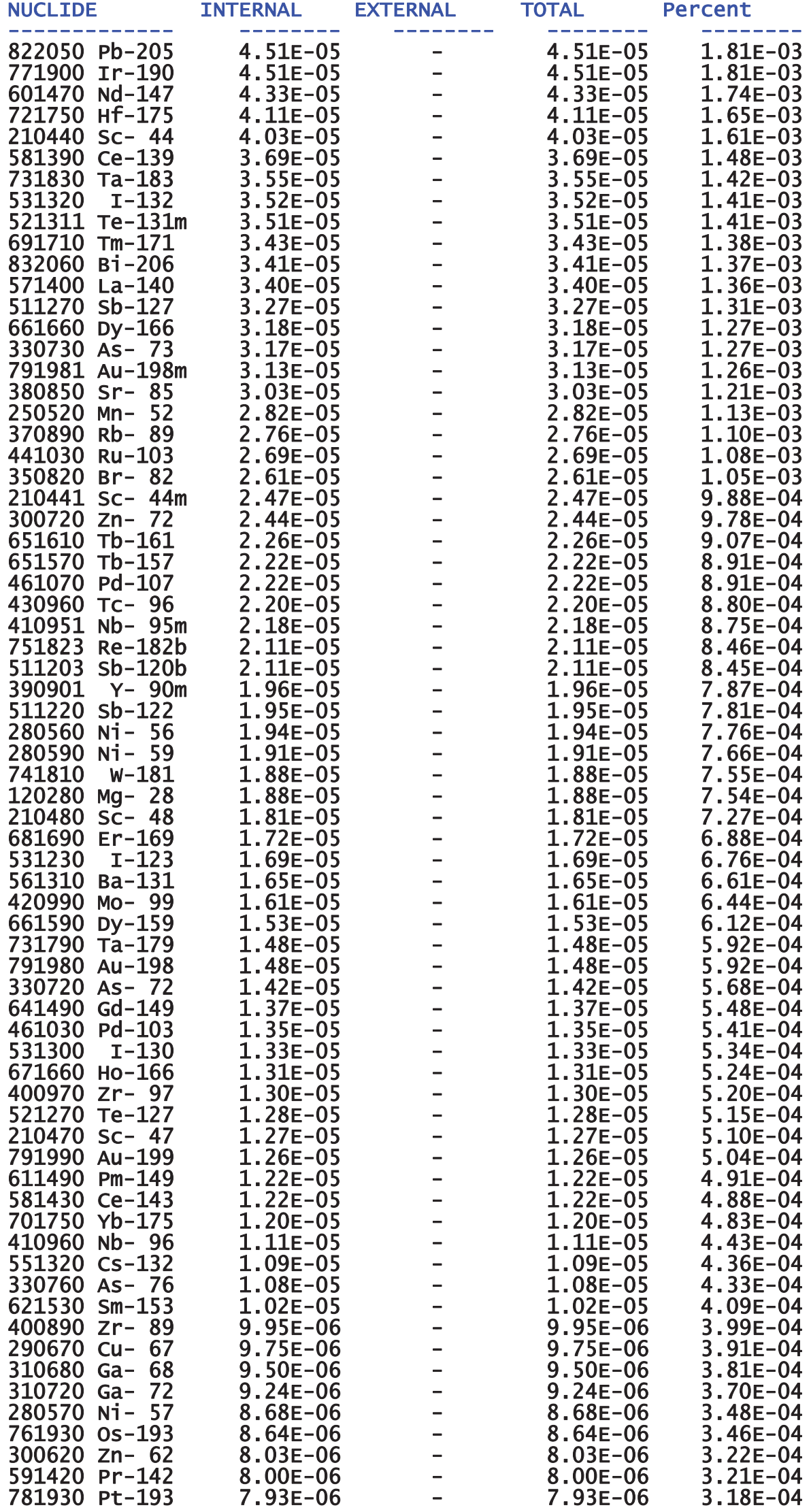


SORTED BY PERCENT OF EFFECTIVE DOSE

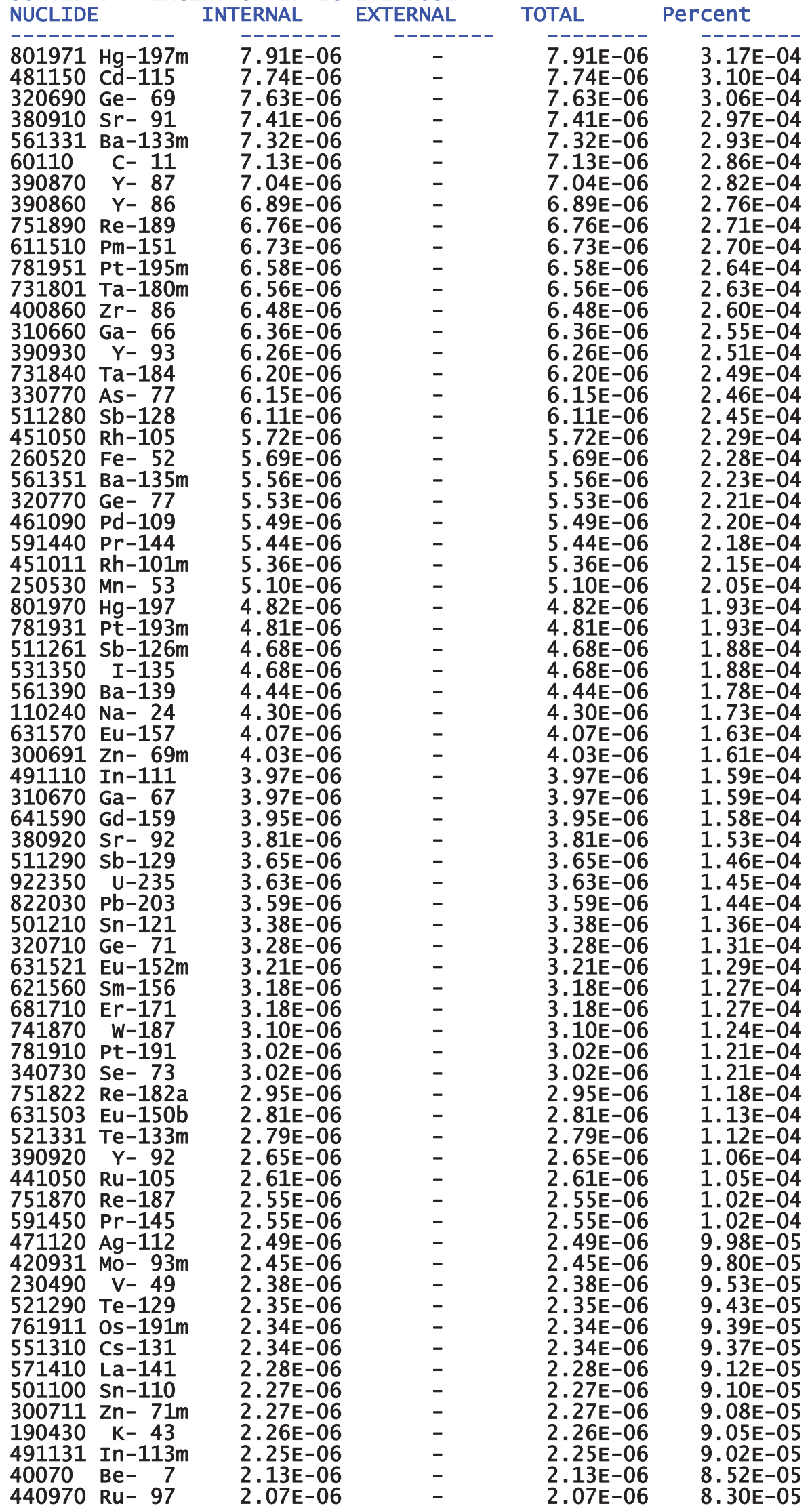


SORTED BY PERCENT OF EFFECTIVE DOSE

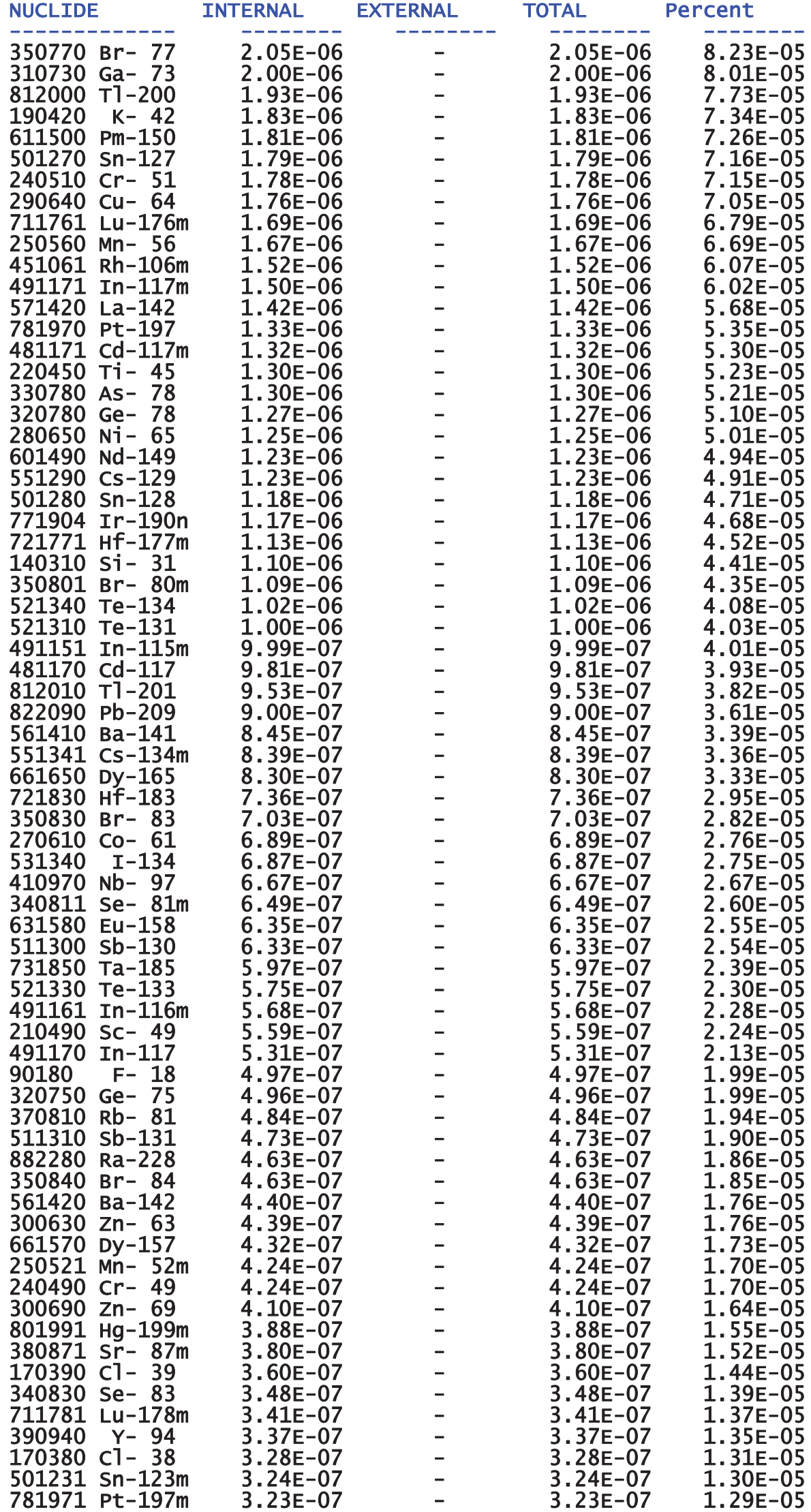


SORTED BY PERCENT OF EFFECTIVE DOSE

\begin{tabular}{|c|c|c|c|c|c|}
\hline NUCLIDE & & INTERNAL & EXTERNAL & TOTAL & Percent \\
\hline 431040 & TC-104 & $2.91 \mathrm{E}-07$ & - & $2.91 \mathrm{E}-07$ & $1.16 \mathrm{E}-05$ \\
\hline 471150 & $\mathrm{Aq}-115$ & $2.88 \mathrm{E}-07$ & - & $2.88 \mathrm{E}-07$ & 05 \\
\hline 430991 & TC- $99 \mathrm{~m}$ & $2.87 \mathrm{E}-07$ & - & $2.87 \mathrm{E}-07$ & $15 \mathrm{E}-05$ \\
\hline 711780 & Lu-178 & $2.87 \mathrm{E}-07$ & - & $2.87 E-07$ & $1.15 \mathrm{E}-05$ \\
\hline 390950 & $Y-95$ & $2.52 E-07$ & - & $2.52 E-07$ & $1.01 \mathrm{E}-05$ \\
\hline 270581 & $\mathrm{Co}-58 \mathrm{~m}$ & $2.47 \mathrm{E}-07$ & - & $2.47 \mathrm{E}-07$ & $9.90 \mathrm{E}-06$ \\
\hline 40 & $\mathrm{~Pa}-234$ & $2.40 \mathrm{E}-07$ & - & $2.40 \mathrm{E}-07$ & $9.62 \mathrm{E}-06$ \\
\hline 571430 & La-143 & $2.40 \mathrm{E}-07$ & - & $2.40 \mathrm{E}-07$ & $9.62 \mathrm{E}-06$ \\
\hline 511170 & sb-117 & $2.37 \mathrm{E}-07$ & - & $2.37 \mathrm{E}-07$ & $51 \mathrm{E}-06$ \\
\hline 421010 & Mo-101 & $2.21 \mathrm{E}-07$ & - & $2.21 E-07$ & $8.88 \mathrm{E}-06$ \\
\hline 310 & Se- 81 & $2.20 \mathrm{E}-07$ & - & $2.20 \mathrm{E}-07$ & 81E-06 \\
\hline & $\mathrm{Sb}-128 \mathrm{~m}$ & $2.11 \mathrm{E}-07$ & - & $2.11 E-07$ & $46 E-06$ \\
\hline 351 & Cs $-135 \mathrm{~m}$ & $2.01 E-07$ & - & $2.01 E-07$ & $8.07 E-06$ \\
\hline 491191 & In-119m & $1.85 \mathrm{E}-07$ & - & $1.85 \mathrm{E}-07$ & 7. $42 \mathrm{E}-06$ \\
\hline 21 & $\mathrm{Ta}-182 \mathrm{~m}$ & $1.85 \mathrm{E}-07$ & - & $1.85 \mathrm{E}-07$ & 7.40E-06 \\
\hline 50 & $\mathrm{Sm}-$ & $1.80 \mathrm{E}-07$ & - & 1.80 & 7. $23 \mathrm{E}-06$ \\
\hline & Rh- & $1.77 \mathrm{E}-07$ & - & 1.77 & $7.08 \mathrm{E}-06$ \\
\hline 70 & Rh-107 & $1.75 \mathrm{E}-07$ & - & $1.75 \mathrm{E}-07$ & $7.01 \mathrm{E}-06$ \\
\hline 621 & $\mathrm{Co}-62 \mathrm{~m}$ & $1.72 \mathrm{E}-07$ & - & $1.72 \mathrm{E}-07$ & $6.89 \mathrm{E}-06$ \\
\hline 060 & $\mathrm{Ag}-106$ & $1.67 \mathrm{E}-07$ & - & $1.67 \mathrm{E}-07$ & $6.70 \mathrm{E}-06$ \\
\hline 700 & Ga- 70 & $1.60 \mathrm{E}-07$ & - & $1.60 \mathrm{E}-07$ & $6.41 \mathrm{E}-06$ \\
\hline 010 & Tc-101 & $1.57 \mathrm{E}-07$ & - & $1.57 \mathrm{E}-07$ & $30 E-06$ \\
\hline 470 & $\mathrm{Pr}-147$ & $1.56 \mathrm{E}-07$ & - & $1.56 \mathrm{E}-07$ & $6.26 \mathrm{E}-06$ \\
\hline 90 & Eu-149 & $1.49 \mathrm{E}-07$ & - & $1.49 \mathrm{E}-07$ & $5.97 \mathrm{E}-06$ \\
\hline 671641 & Ho-164m & $1.42 \mathrm{E}-07$ & - & 1.42 & $5.68 \mathrm{E}-06$ \\
\hline 531280 & I-128 & $1.38 \mathrm{E}-07$ & - & 1.38 & $5.52 \mathrm{E}-06$ \\
\hline & $Y-91 m$ & $1.37 \mathrm{E}-07$ & 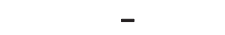 & $1.37 \mathrm{~b}$ & $49 \mathrm{E}$ \\
\hline 00 & $\mathrm{Br}-80$ & $1.37 \mathrm{E}-07$ & - & $1.37 \mathrm{E}-07$ & $5.49 \mathrm{E}-06$ \\
\hline 601510 & Nd-151 & 1. 34E-07 & - & 1.34 & $5.36 \mathrm{E}-06$ \\
\hline & Ir $-190 \mathrm{~m}$ & 1.32 & - & 1.32 & $5.28 \mathrm{E}-06$ \\
\hline & $\mathrm{Re}-188 \mathrm{~m}$ & -07 & - & & $94 \mathrm{E}-06$ \\
\hline 60 & Тa-186 & 1.2 & - & 1.22 & $4.89 \mathrm{E}-06$ \\
\hline & Ho-164 & 1.2 & - & 7 & $4.81 \mathrm{E}-06$ \\
\hline & Tc- $96 \mathrm{~m}$ & 9.3 & - & 9.39 & $3.76 \mathrm{E}-06$ \\
\hline & os $-189 m$ & 7. & - & 7. & $.06 \mathrm{E}$ \\
\hline & In-110m & 7. & - & 7. & $00 \mathrm{E}-$ \\
\hline & $\mathrm{Co}-60 \mathrm{~m}$ & 7. & - & 7.07 & $83 E-06$ \\
\hline & Nd-141 & & - & & $8 \mathrm{E}$ \\
\hline & $S r-85 m$ & 5. & - & & $2.24 \mathrm{E}-06$ \\
\hline & W-179 & 1. & - & & \\
\hline & Th- & 2 & - & & O०F \\
\hline & $A C-228$ & 2. & - & & $9.59 \mathrm{E}-09$ \\
\hline & U-234 & 1 & - & & 1. $54 \mathrm{E}-09$ \\
\hline & $\mathrm{Pa}-231$ & & - & & $1.41 \mathrm{E}-1$ \\
\hline & $\mathrm{Nb}-$ & $\perp$ & - & & 115 \\
\hline & Ra-226 & 7. & - & 7. & $2.87 \mathrm{E}-14$ \\
\hline 10 & Th- & - & - & & $61 \mathrm{E}-1$ \\
\hline & ALS & $\begin{array}{c}\mathrm{C}+0 \\
-1\end{array}$ & - & $\angle . J U E+U 0$ & $1.00 \mathrm{E}+0$ \\
\hline
\end{tabular}


SUM OF CONTRIBUTIONS TO THE EFFECTIVE DOSE (rem) DOWNWIND DISTANCE $=5.00 \mathrm{E}+02(\mathrm{~m})$

SORTED BY PERCENT OF EFFECTIVE DOSE

NUCLIDE

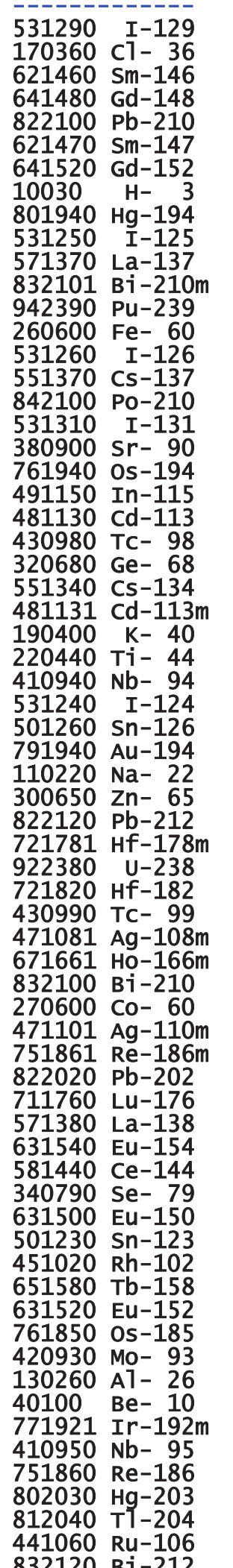

INTERNAL

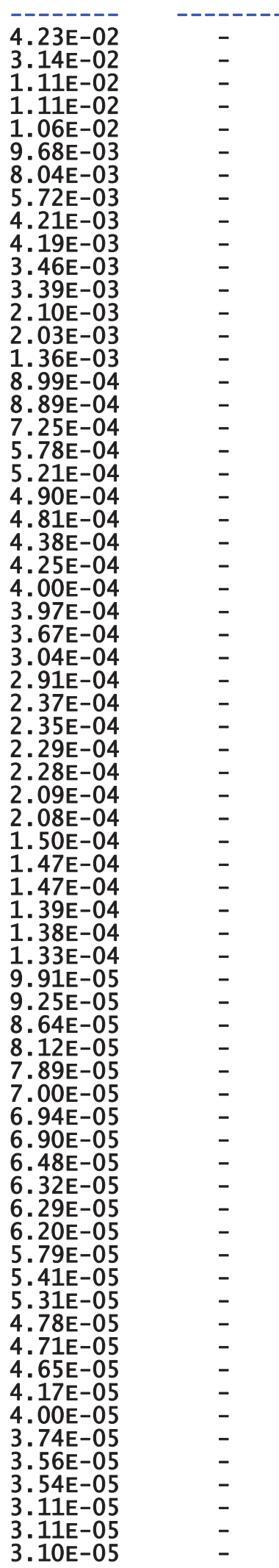

TOTAL

4. $23 \mathrm{E}-02$

$3.14 \mathrm{E}-02$

1.11E-02

1. 11E-02

$1.06 \mathrm{E}-02$

9. $68 \mathrm{E}-03$

8. $04 \mathrm{E}-03$

$5.72 \mathrm{E}-03$

$4.21 \mathrm{E}-03$

4. $19 \mathrm{E}-03$

3. $46 \mathrm{E}-03$

$3.39 \mathrm{E}-03$

2. $10 \mathrm{E}-03$

2. $03 \mathrm{E}-03$

1. 36E-03

$8.99 \mathrm{E}-04$

$8.89 \mathrm{E}-04$

7. 25E-04

$5.78 \mathrm{E}-04$

5. 21E-04

4. $90 \mathrm{E}-04$

4. 81E-04

4. 38E-04

4. 25E-04

4. 00E-04

3.97E-04

3.67E-04

3. 04E-04

2. $91 \mathrm{E}-04$

2.37E-04

2. 35E-04

2. $29 \mathrm{E}-04$

2. 28E-04

2.09E-04

2.08E-04

1. $50 \mathrm{E}-04$

1. 47E-04

1. 47E-04

1.39E-04

1. 38E-04

1. 33E-04

$9.91 \mathrm{E}-05$

9. $25 \mathrm{E}-05$

8.64E-05

8.12E-05

$7.89 \mathrm{E}-05$

7. $00 \mathrm{E}-05$

$6.94 \mathrm{E}-05$

6. $90 \mathrm{E}-05$

$6.48 \mathrm{E}-05$

$6.32 \mathrm{E}-05$

$6.29 \mathrm{E}-05$

$6.20 \mathrm{E}-05$

$5.79 \mathrm{E}-05$

5. 41E-05

5. 31E-05

$4.78 \mathrm{E}-05$

$4.71 \mathrm{E}-05$

$4.65 \mathrm{E}-05$

4.17E-05

4. $00 \mathrm{E}-05$

3. 74E-05

3. 56E-05

3.54E-05

3. 11E-05

3. 11E-05

3. 10E-05
Percent

2. $60 \mathrm{E}+01$

1. $93 \mathrm{E}+01$

$6.80 \mathrm{E}+00$

$6.79 \mathrm{E}+00$

6. $50 \mathrm{E}+00$

$5.95 \mathrm{E}+00$

4. $94 \mathrm{E}+00$

$3.51 \mathrm{E}+00$

2. $58 \mathrm{E}+00$

2. $57 \mathrm{E}+00$

2. $13 \mathrm{E}+00$

2. $08 \mathrm{E}+00$

1. $29 \mathrm{E}+00$

1. $25 \mathrm{E}+00$

8. 37E-01

5. $52 \mathrm{E}-01$

5. 46E-01

4. $45 \mathrm{E}-01$

3. $55 \mathrm{E}-01$

3. $20 \mathrm{E}-01$

3. 01E-01

2. $95 \mathrm{E}-01$

2. 69E-01

2. 61E-01

2. $46 \mathrm{E}-01$

2. 44E-01

2. 26E-01

$1.86 \mathrm{E}-01$

1. $79 \mathrm{E}-01$

1. 46E-01

1. $44 \mathrm{E}-01$

1. 41E-01

1. $40 \mathrm{E}-01$

1. $28 \mathrm{E}-01$

1. $28 \mathrm{E}-01$

9. 19E-02

9. $03 \mathrm{E}-02$

9. 03E-02

8. 53E-02

8. 48E-02

8. 19E-02

6. 09E-02

5. 68E-02

5. 31E-02

4. 99E-02

4. 84E-02

4. 30E-02

4. $26 \mathrm{E}-02$

4. 24E-02

3. 98E-02

$3.88 \mathrm{E}-02$

$3.86 \mathrm{E}-02$

3. 81E-02

3. 55E-02

3. 32E-02

3. $26 \mathrm{E}-02$

2. 93E-02

2. 89E-02

$2.85 \mathrm{E}-02$

2. $56 \mathrm{E}-02$

2. $46 \mathrm{E}-02$

2. 30E-02

2. $19 \mathrm{E}-02$

2. 17E-02

1. $91 \mathrm{E}-02$

1. $91 \mathrm{E}-02$

1. 90E-02 
DOWNWIND DISTANCE $=5.00 \mathrm{E}+02(\mathrm{~m})$

SORTED BY PERCENT OF EFFECTIVE DOSE

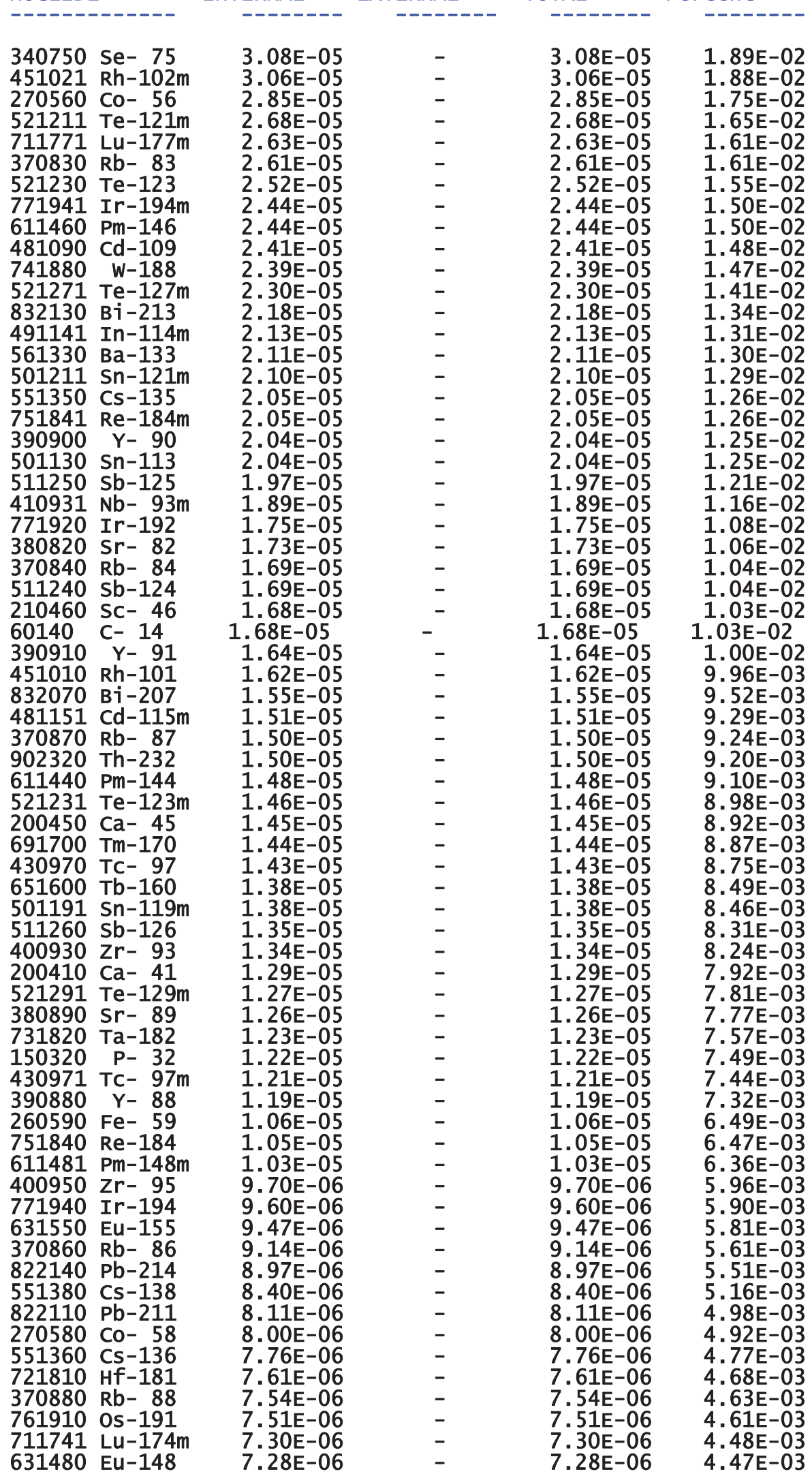


DOWNWIND DISTANCE $=5.00 \mathrm{E}+02(\mathrm{~m})$

SORTED BY PERCENT OF EFFECTIVE DOSE

\section{NUCLIDE}

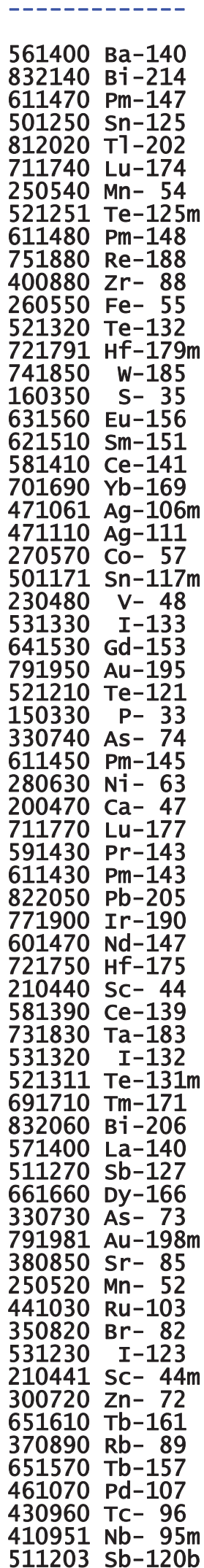
INTERNAL

\section{7.}

$6.96 \mathrm{E}-06$

6. $81 \mathrm{E}-06$

6. 40E-06

6. $08 \mathrm{E}-06$

6. $04 \mathrm{E}-06$

$5.87 \mathrm{E}-06$

$5.74 \mathrm{E}-06$

$5.72 \mathrm{E}-06$

5. 68E-06

$5.52 \mathrm{E}-06$

5. 36E-06

5. 31E-06

5. 24E-06

5. $06 \mathrm{E}-06$

$4.75 \mathrm{E}-06$

4. $43 \mathrm{E}-06$

4. 38E-06

4.19E-06

4. $14 \mathrm{E}-06$

4. $01 \mathrm{E}-06$

3. $96 \mathrm{E}-06$

3. $94 \mathrm{E}-06$

3. 89E-06

3. $85 \mathrm{E}-06$

3. $61 \mathrm{E}-06$

3. $58 \mathrm{E}-06$

3. $25 \mathrm{E}-06$

3. 24E-06

3. 21E-06

3. 21E-06

3. 16E-06

3. 13E-06

3. 07E-06

3. $06 \mathrm{E}-06$

2. $96 \mathrm{E}-06$

2. $96 \mathrm{E}-06$

2. 84E-06

2. 69E-06

2. 68E-06

2. 42E-06

2. 33E-06

2. 31E-06

2. 29E-06

2. 25E-06

2. $23 \mathrm{E}-06$

2. 23E-06

2. 15E-06

2. $08 \mathrm{E}-06$

2. $08 \mathrm{E}-06$

2.05E-06

$1.98 \mathrm{E}-06$

1.85E-06

1.77E-06

1. $71 \mathrm{E}-06$

1. $65 \mathrm{E}-06$

1. $62 \mathrm{E}-06$

1. $60 \mathrm{E}-06$

1. $48 \mathrm{E}-06$

1. 47E-06

$1.46 \mathrm{E}-06$

$1.46 \mathrm{E}-06$

1. 44E-06

1. $43 \mathrm{E}-06$

1. 38E-06
EXTERNAL
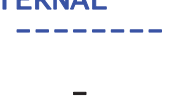

$-$

$-$

$-$

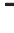

-

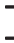

-

-

-

-

$-$

$-$

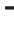

-

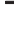

$-$

$-$

-

-

$-$

$-$

-

$-$

-

$-$

$-$

$-$

$-$

$-$

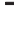

$-$

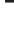

$-$

$-$

-

$-$

-

$-$

-

$-$

-

$-$

-

-

$-$

-

$-$

$-$

-
TOTAL

7.26E-06

7. $10 \mathrm{E}-06$

$6.96 \mathrm{E}-06$

$6.87 \mathrm{E}-06$

$6.81 \mathrm{E}-06$

$6.40 \mathrm{E}-06$

$6.08 \mathrm{E}-06$

6.04E-06

5.87E-06

$5.74 \mathrm{E}-06$

$5.72 \mathrm{E}-06$

$5.68 \mathrm{E}-06$

5. $52 \mathrm{E}-06$

$5.36 \mathrm{E}-06$

5. 31E-06

5. $24 \mathrm{E}-06$

5. $06 \mathrm{E}-06$

4.75E-06

4. 43E-06

4. 38E-06

4. 19E-06

4. 14E-06

4.01E-06

3. $96 \mathrm{E}-06$

3. 94E-06

3. 89E-06

3. 85E-06

3. $61 \mathrm{E}-06$

3. 58E-06

3. $25 \mathrm{E}-06$

3. 24E-06

3. 21E-06

3. 21E-06

3. $16 \mathrm{E}-06$

3. 13E-06

3. 07E-06

3.06E-06

2.96E-06

2. 96E-06

2. 84E-06

2. 69E-06

2. 68E-06

2. 42E-06

2. 33E-06

2. 31E-06

2. 29E-06

$2.25 \mathrm{E}-06$

2. $23 \mathrm{E}-06$

2. 23E-06

2. $15 \mathrm{E}-06$

2. $08 \mathrm{E}-06$

2. $08 \mathrm{E}-06$

2. $05 \mathrm{E}-06$

$1.98 \mathrm{E}-06$

1. 85E-06

1.77E-06

1. 71E-06

1. 65E-06

1. 62E-06

1. 60E-06

1. $48 \mathrm{E}-06$

$1.47 \mathrm{E}-06$

$1.46 \mathrm{E}-06$

$1.46 \mathrm{E}-06$

1. 44E-06

1. $43 \mathrm{E}-06$

1. $38 \mathrm{E}-06$
Percent

$4.46 \mathrm{E}-03$

4. 36E-03

$4.27 \mathrm{E}-03$

4. $22 \mathrm{E}-03$

$4.18 \mathrm{E}-03$

3. $93 \mathrm{E}-03$

$3.73 \mathrm{E}-03$

$3.71 \mathrm{E}-03$

3. $61 \mathrm{E}-03$

3. $53 \mathrm{E}-03$

$3.52 \mathrm{E}-03$

3. $49 \mathrm{E}-03$

3. $39 \mathrm{E}-03$

$3.29 \mathrm{E}-03$

3. $26 \mathrm{E}-03$

$3.22 \mathrm{E}-03$

3. $11 \mathrm{E}-03$

$2.92 \mathrm{E}-03$

$2.72 \mathrm{E}-03$

2. $69 \mathrm{E}-03$

$2.57 \mathrm{E}-03$

2. $55 \mathrm{E}-03$

$2.46 \mathrm{E}-03$

2. $44 \mathrm{E}-03$

2. $42 \mathrm{E}-03$

2. $39 \mathrm{E}-03$

2. 37E-03

2. $22 \mathrm{E}-03$

2. $20 \mathrm{E}-03$

2. $00 \mathrm{E}-03$

$1.99 \mathrm{E}-03$

$1.97 \mathrm{E}-03$

$1.97 \mathrm{E}-03$

$1.94 \mathrm{E}-03$

$1.92 \mathrm{E}-03$

$1.88 \mathrm{E}-03$

$1.88 \mathrm{E}-03$

$1.82 \mathrm{E}-03$

$1.82 \mathrm{E}-03$

$1.74 \mathrm{E}-03$

$1.65 \mathrm{E}-03$

$1.64 \mathrm{E}-03$

1. $49 \mathrm{E}-03$

$1.43 \mathrm{E}-03$

$1.42 \mathrm{E}-03$

$1.41 \mathrm{E}-03$

1. $38 \mathrm{E}-03$

$1.37 \mathrm{E}-03$

1. $37 \mathrm{E}-03$

1. $32 \mathrm{E}-03$

$1.28 \mathrm{E}-03$

$1.28 \mathrm{E}-03$

$1.26 \mathrm{E}-03$

$1.22 \mathrm{E}-03$

$1.13 \mathrm{E}-03$

$1.09 \mathrm{E}-03$

$1.05 \mathrm{E}-03$

1. 01E-03

9. $92 \mathrm{E}-04$

9. 82E-04

$9.11 \mathrm{E}-04$

9. $03 \mathrm{E}-04$

8.96E-04

8. $96 \mathrm{E}-04$

8. 85E-04

$8.79 \mathrm{E}-04$

8. 49E-04 
DOWNWIND DISTANCE $=5.00 \mathrm{E}+02(\mathrm{~m})$ SORTED BY PERCENT OF EFFECTIVE DOSE

\section{NUCLIDE}

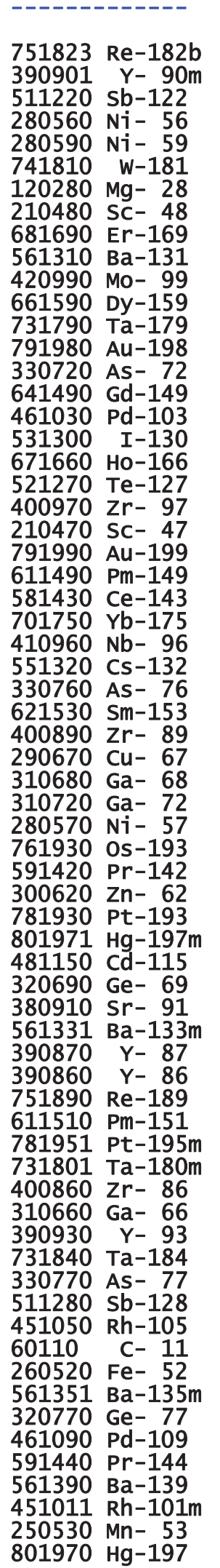
INTERNAL

$-----$

\section{$1.38 E-06$}

1. $29 \mathrm{E}-06$

1. $28 \mathrm{E}-06$

1.27E-06

$1.25 \mathrm{E}-06$

$1.24 \mathrm{E}-06$

$1.23 \mathrm{E}-06$

$1.19 \mathrm{E}-06$

$1.12 \mathrm{E}-06$

$1.08 \mathrm{E}-06$

$1.05 \mathrm{E}-06$

1.00E-06

9. $70 \mathrm{E}-07$

9.69E-07

9. $28 \mathrm{E}-07$

8.97E-07

$8.85 \mathrm{E}-07$

8.69E-07

$8.58 \mathrm{E}-07$

$8.48 \mathrm{E}-07$

8.47E-07

8. 35E-07

8.24E-07

8.03E-07

7.97E-07

$7.89 \mathrm{E}-07$

7.23E-07

$7.13 \mathrm{E}-07$

$7.06 \mathrm{E}-07$

6. $68 \mathrm{E}-07$

$6.52 \mathrm{E}-07$

$6.39 \mathrm{E}-07$

$6.23 \mathrm{E}-07$

$6.06 \mathrm{E}-07$

$5.68 \mathrm{E}-07$

$5.66 \mathrm{E}-07$

5.23E-07

$5.23 \mathrm{E}-07$

5.20E-07

$5.17 \mathrm{E}-07$

5.07E-07

5.00E-07

$4.82 \mathrm{E}-07$

4.79E-07

4. 61E-07

4. 52E-07

4.42E-07

4.41E-07

4. 32E-07

4. 30E-07

4. 23E-07

4.14E-07

4.11E-07

4.03E-07

4. $03 \mathrm{E}-07$

3. 97E-07

$3.75 \mathrm{E}-07$

3.73E-07

3. 70E-07

3. 64E-07

3. $60 \mathrm{E}-07$

$3.58 \mathrm{E}-07$

3. 58E-07

3. $54 \mathrm{E}-07$

3. 51E-07

3. $35 \mathrm{E}-07$

3.16E-07
EXTERNAL

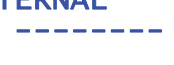

$-$

$-$

-

$-$

-

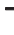

$-$

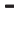

$-$

$-$

-

$-$

-

$-$

-

$-$

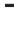

$-$

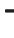

-

$-$

-

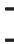

-

$-$

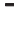

$-$

$-$

-

-

-

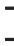

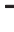

$-$

$-$

-

$-$

-

$-$

-

$-$

$-$

$-$

-

$-$

$-$

$-$

$-$

-

$-$

-

$-$

-

-

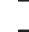

-

$-$

-

-

TOTAL

1. 38E-06

$1.29 \mathrm{E}-06$

$1.28 \mathrm{E}-06$

1.27E-06

$1.25 \mathrm{E}-06$

$1.24 \mathrm{E}-06$

1. $23 \mathrm{E}-06$

1. 19E-06

1. $12 \mathrm{E}-06$

1.08E-06

$1.05 \mathrm{E}-06$

1. $00 \mathrm{E}-06$

9. 70E-07

9.69E-07

9. 28E-07

8.97E-07

$8.85 \mathrm{E}-07$

8.69E-07

8. 58E-07

8. 48E-07

8.47E-07

8. 35E-07

8. 24E-07

8.03E-07

7.97E-07

7. 89E-07

7. 23E-07

7.13E-07

7.06E-07

6. 68E-07

6. 52E-07

6. 39E-07

6. 23E-07

6. 06E-07

5. 68E-07

5. 66E-07

5. 23E-07

5. 23E-07

5. 20E-07

5. 17E-07

5. 07E-07

5. 00E-07

4. 82E-07

4.79E-07

4. 61E-07

4. 52E-07

4. 42E-07

4. $41 \mathrm{E}-07$

4. 32E-07

4. 30E-07

4. 23E-07

4. 14E-07

4. $11 \mathrm{E}-07$

4. 03E-07

4.03E-07

3.97E-07

3. 75E-07

3. 73E-07

3. 70E-07

3. 64E-07

3. $60 \mathrm{E}-07$

3. 58E-07

3. 58E-07

3. 54E-07

3. 51E-07

3. 35E-07

3. 16E-07
Percent

8. $49 \mathrm{E}-04$

7.91E-04

7. 84E-04

7. 80E-04

$7.70 \mathrm{E}-04$

7. 59E-04

7. 56E-04

7. 30E-04

6. $91 \mathrm{E}-04$

6. 64E-04

6. $46 \mathrm{E}-04$

6. $15 \mathrm{E}-04$

5. $96 \mathrm{E}-04$

5.95E-04

5.70E-04

5. 51E-04

5. 44E-04

5. 34E-04

5. 27E-04

5. 21E-04

5. 20E-04

5. 13E-04

$5.06 \mathrm{E}-04$

4. $93 \mathrm{E}-04$

4. $90 \mathrm{E}-04$

4. 85E-04

4. 44E-04

4. $38 \mathrm{E}-04$

4. 34E-04

4. 10E-04

4. 00E-04

3. 92E-04

3. 83E-04

3. $72 \mathrm{E}-04$

3. 49E-04

3. 47E-04

3. $21 \mathrm{E}-04$

3. 21E-04

3. 20E-04

3. $18 \mathrm{E}-04$

3. 11E-04

3. 07E-04

2. $96 \mathrm{E}-04$

2. $94 \mathrm{E}-04$

2. 83E-04

$2.77 \mathrm{E}-04$

2. $72 \mathrm{E}-04$

2. 71E-04

2. $65 \mathrm{E}-04$

2. 64E-04

2. 60E-04

2. 54E-04

2. 52E-04

2. $48 \mathrm{E}-04$

$2.48 \mathrm{E}-04$

2. $44 \mathrm{E}-04$

2. 30E-04

2. 29E-04

2. 27E-04

2. 23E-04

2. 21E-04

2. $20 \mathrm{E}-04$

2. $20 \mathrm{E}-04$

2. 17E-04

2. $16 \mathrm{E}-04$

$2.06 \mathrm{E}-04$
$1.94 \mathrm{E}-04$ 
DOWNWIND DISTANCE $=5.00 \mathrm{E}+02(\mathrm{~m})$

SORTED BY PERCENT OF EFFECTIVE DOSE

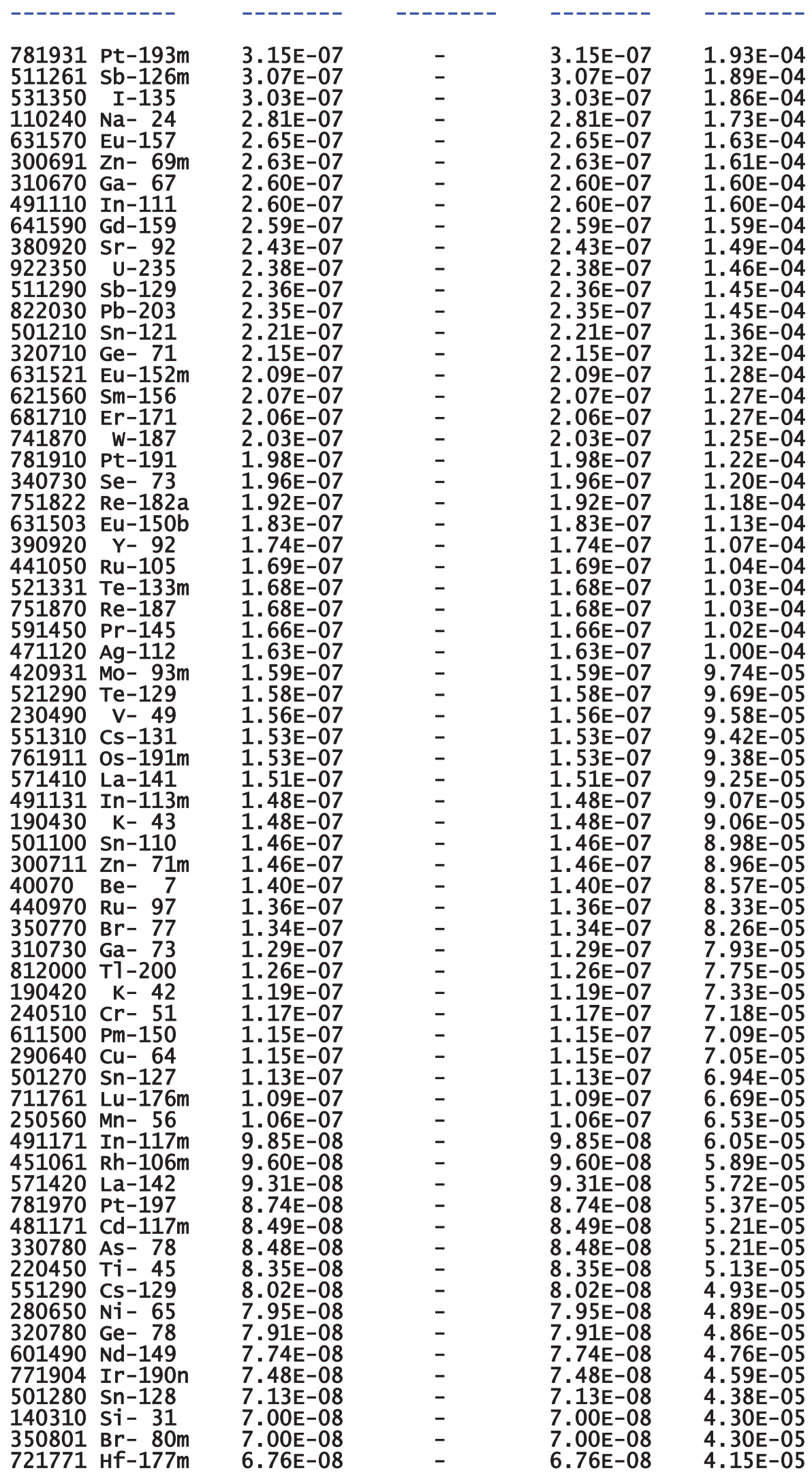


DOWNWIND DISTANCE $=5.00 \mathrm{E}+02(\mathrm{~m})$

SORTED BY PERCENT OF EFFECTIVE DOSE

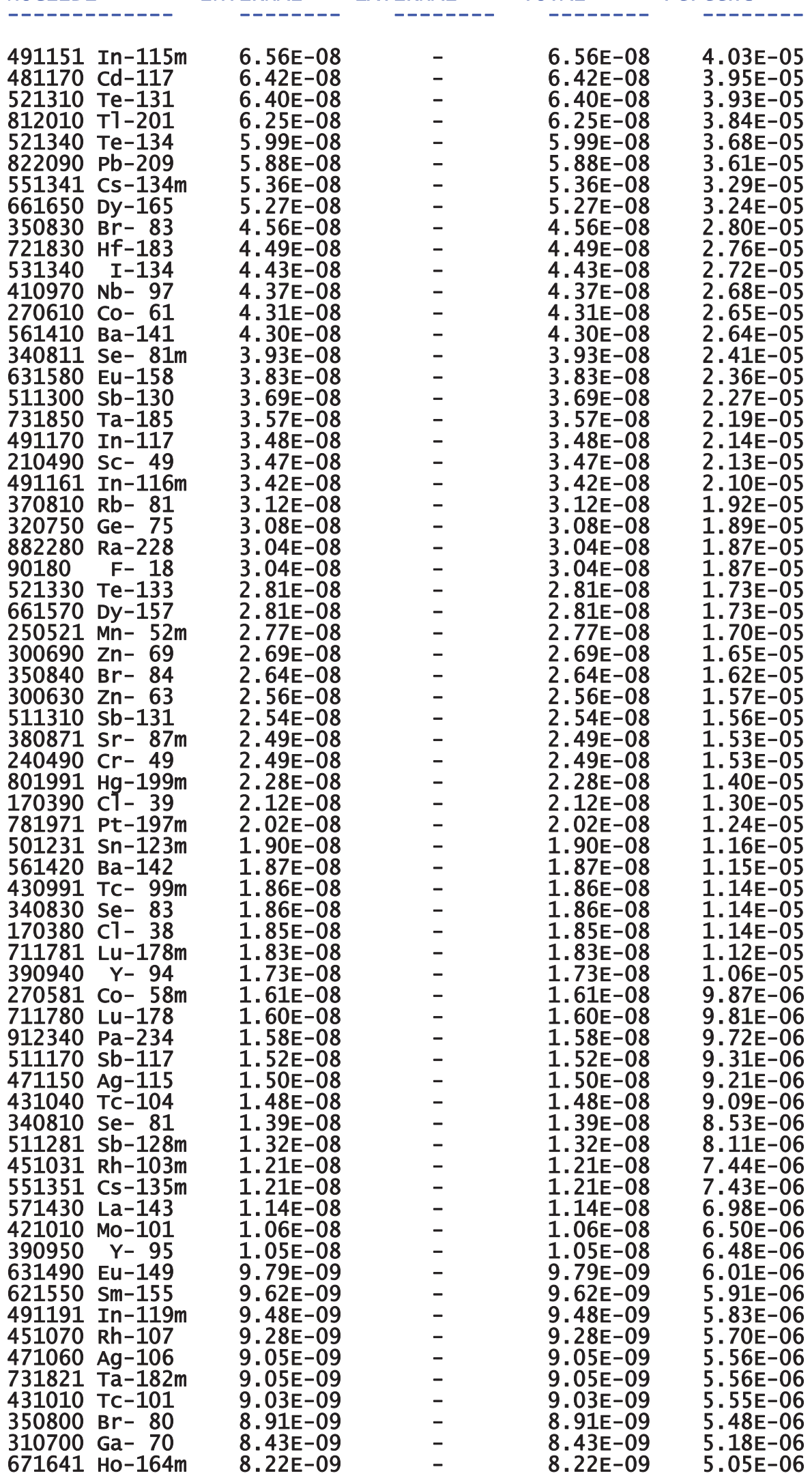




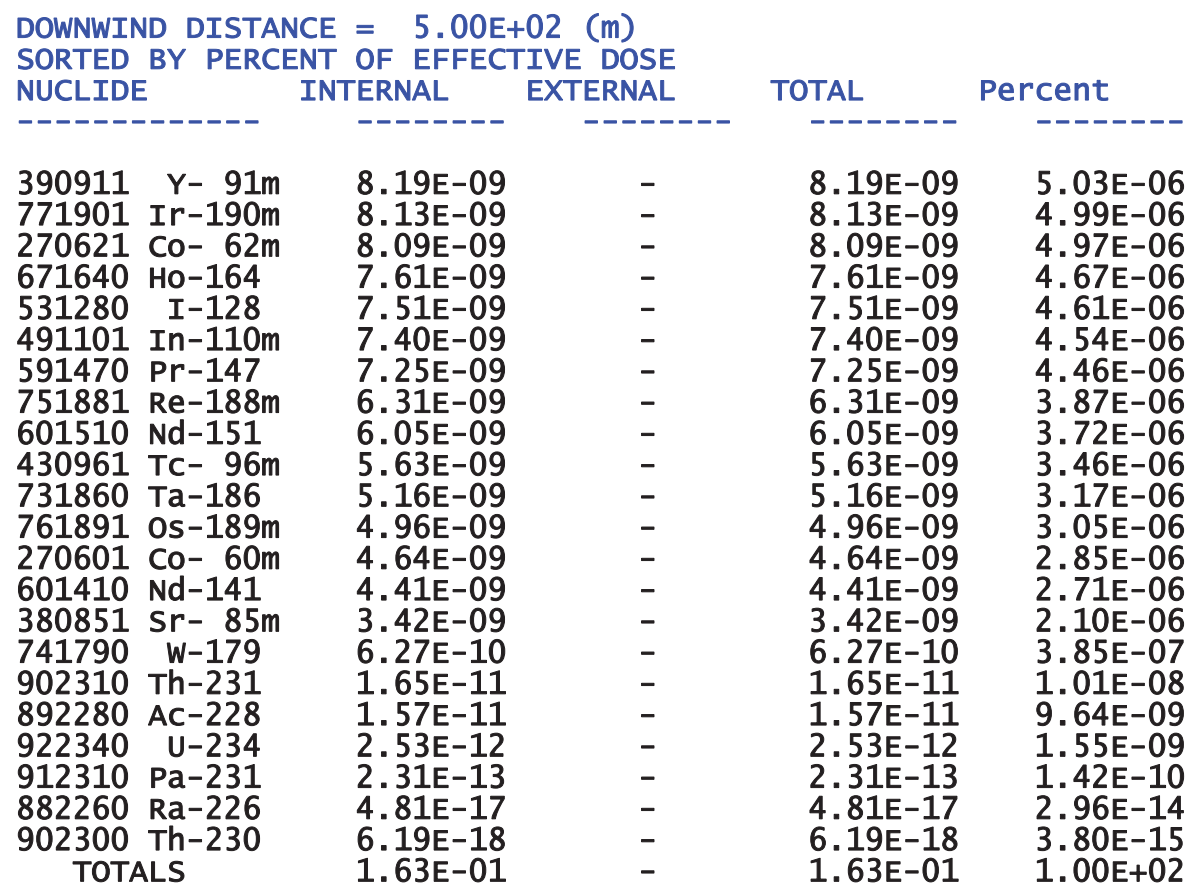


SUM OF CONTRIBUTIONS TO THE EFFECTIVE DOSE (rem) DOWNWIND DISTANCE $=1.00 \mathrm{E}+03(\mathrm{~m})$

SORTED BY PERCENT OF EFFECTIVE DOSE

NUCLIDE

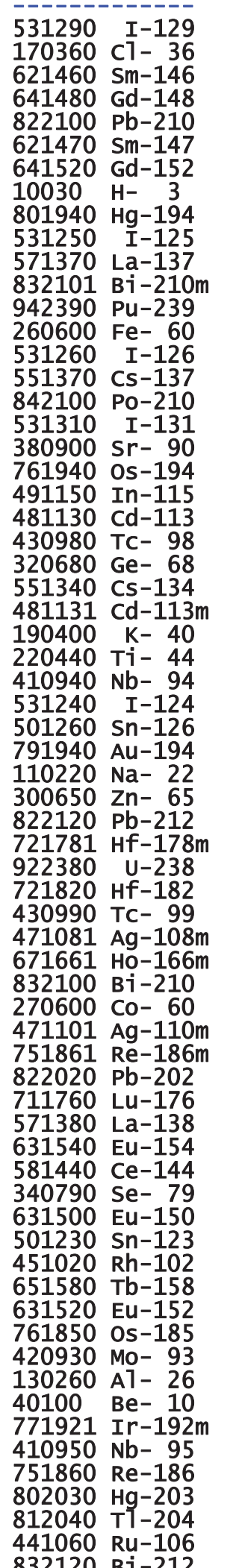

INTERNAL

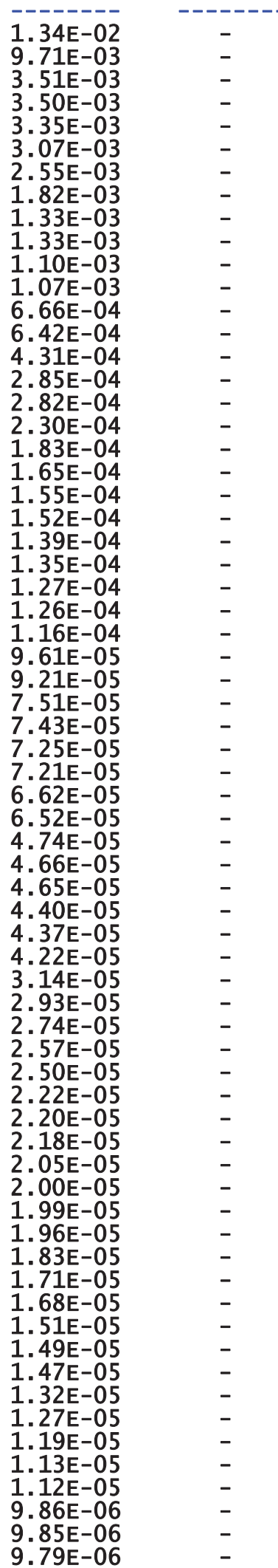

TOTAL

$1.34 \mathrm{E}-02$

$9.71 \mathrm{E}-03$

3. $51 \mathrm{E}-03$

3. $50 \mathrm{E}-03$

$3.35 \mathrm{E}-03$

$3.07 \mathrm{E}-03$

2. $55 \mathrm{E}-03$

$1.82 \mathrm{E}-03$

$1.33 \mathrm{E}-03$

$1.33 \mathrm{E}-03$

$1.10 \mathrm{E}-03$

$1.07 \mathrm{E}-03$

6. 66E-04

$6.42 \mathrm{E}-04$

4. 31E-04

$2.85 \mathrm{E}-04$

2. 82E-04

2. 30E-04

$1.83 \mathrm{E}-04$

$1.65 \mathrm{E}-04$

1. $55 \mathrm{E}-04$

1. 52E-04

$1.39 \mathrm{E}-04$

1. 35E-04

1. $27 \mathrm{E}-04$

1. $26 \mathrm{E}-04$

$1.16 \mathrm{E}-04$

9.61E-05

9. 21E-05

7. $51 \mathrm{E}-05$

7. $43 \mathrm{E}-05$

$7.25 \mathrm{E}-05$

7.21E-05

$6.62 \mathrm{E}-05$

$6.52 \mathrm{E}-05$

4.74E-05

$4.66 \mathrm{E}-05$

4. 65E-05

4. $40 \mathrm{E}-05$

4.37E-05

4. $22 \mathrm{E}-05$

3.14E-05

$2.93 \mathrm{E}-05$

2.74E-05

2. 57E-05

2. 50E-05

2. $22 \mathrm{E}-05$

2. $20 \mathrm{E}-05$

$2.18 \mathrm{E}-05$

2. $05 \mathrm{E}-05$

2.00E-05

$1.99 \mathrm{E}-05$

$1.96 \mathrm{E}-05$

$1.83 \mathrm{E}-05$

$1.71 \mathrm{E}-05$

$1.68 \mathrm{E}-05$

$1.51 \mathrm{E}-05$

$1.49 \mathrm{E}-05$

$1.47 \mathrm{E}-05$

1. 32E-05

1. 27E-05

$1.19 \mathrm{E}-05$

1.13E-05

1. 12E-05

$9.86 \mathrm{E}-06$

9.85E-06

$9.79 \mathrm{E}-06$
Percent

2. $61 \mathrm{E}+01$

$1.89 \mathrm{E}+01$

$6.83 \mathrm{E}+00$

$6.82 \mathrm{E}+00$

$6.53 \mathrm{E}+00$

5. $97 \mathrm{E}+00$

$4.96 \mathrm{E}+00$

3. $54 \mathrm{E}+00$

2. $60 \mathrm{E}+00$

2. $58 \mathrm{E}+00$

2. $13 \mathrm{E}+00$

2. $09 \mathrm{E}+00$

1. $30 \mathrm{E}+00$

1. $25 \mathrm{E}+00$

8. 40E-01

5. 55E-01

5. 49E-01

4. 48E-01

3. $56 \mathrm{E}-01$

3. $22 \mathrm{E}-01$

3.02E-01

2. $96 \mathrm{E}-01$

2. 70E-01

2. 62E-01

2. 47E-01

2. $45 \mathrm{E}-01$

2. 27E-01

1. 87E-01

1. $79 \mathrm{E}-01$

1. 46E-01

1. $45 \mathrm{E}-01$

1. 41E-01

1. $40 \mathrm{E}-01$

1. $29 \mathrm{E}-01$

1. 27E-01

9. 23E-02

9.07E-02

9. 07E-02

8. 57E-02

8. 51E-02

8. $23 \mathrm{E}-02$

6. 11E-02

5. 71E-02

5. 33E-02

5. 01E-02

4. 87E-02

4. 32E-02

4. $28 \mathrm{E}-02$

4. 25E-02

4. $00 \mathrm{E}-02$

3. $90 \mathrm{E}-02$

3. $88 \mathrm{E}-02$

3. 83E-02

3. 57E-02

3. 34E-02

3. $28 \mathrm{E}-02$

2. $95 \mathrm{E}-02$

2. 91E-02

2. 87E-02

2. 57E-02

2. 47E-02

2. 31E-02

2. 20E-02

2. 18E-02

1. $92 \mathrm{E}-02$

1. 92E-02

1. $91 \mathrm{E}-02$ 
DOWNWIND DISTANCE $=1.00 \mathrm{E}+03(\mathrm{~m})$

SORTED BY PERCENT OF EFFECTIVE DOSE

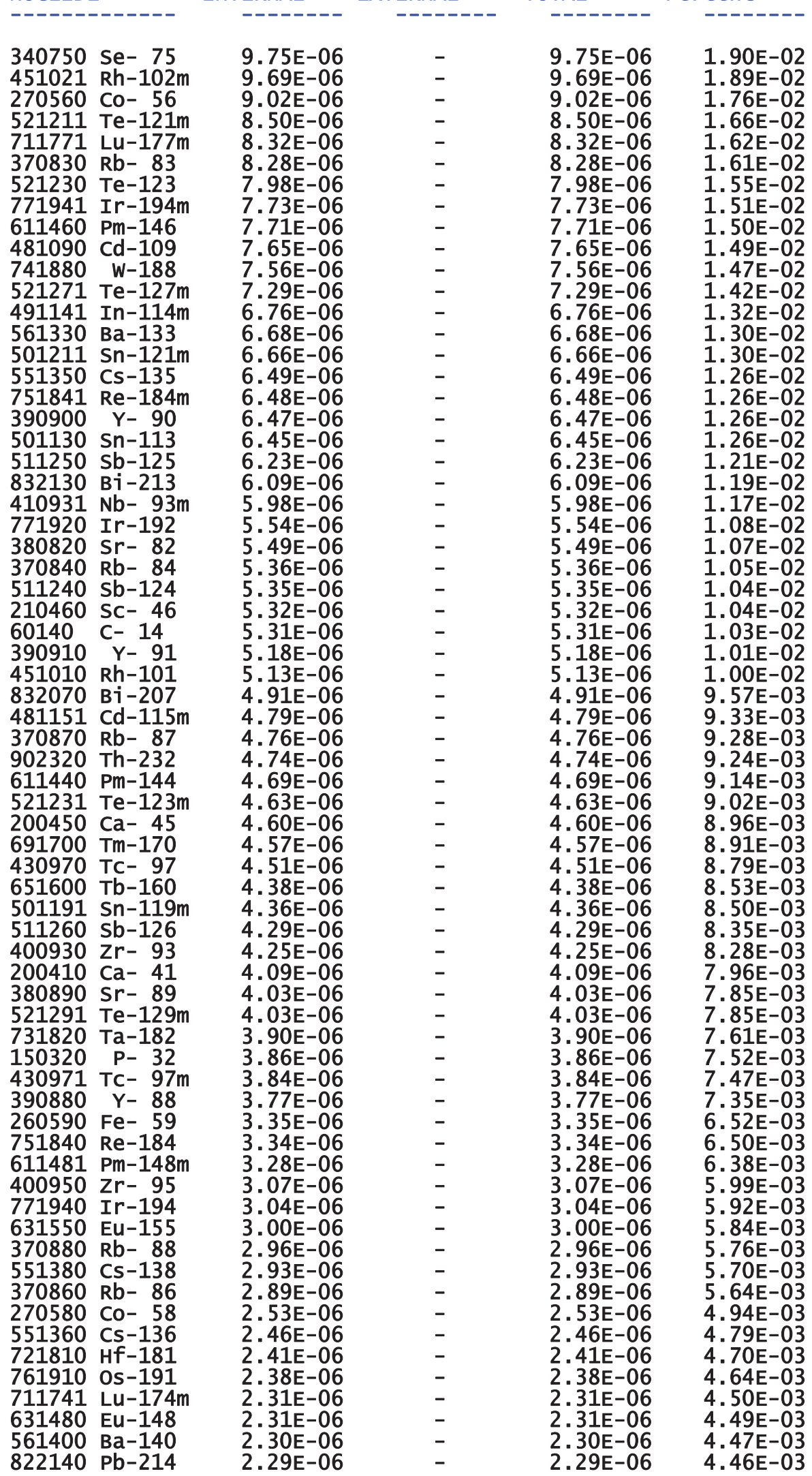


DOWNWIND DISTANCE $=1.00 \mathrm{E}+03(\mathrm{~m})$

SORTED BY PERCENT OF EFFECTIVE DOSE

NUCLIDE

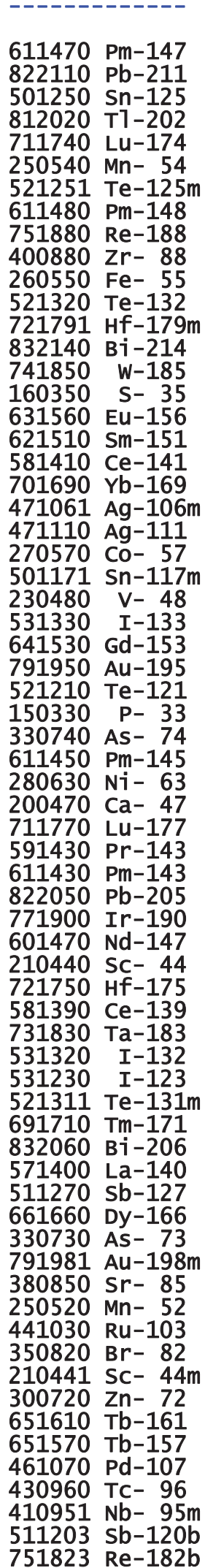
INTERNAL

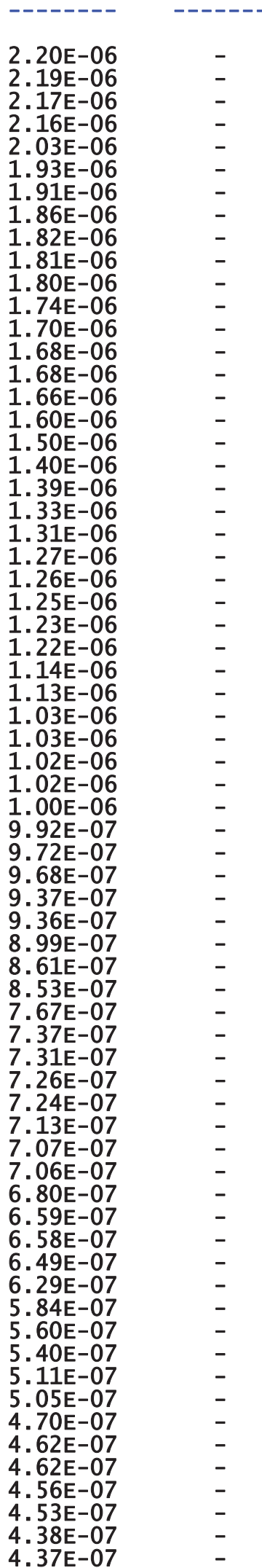

TOTAL

2.20E-06

2. $19 \mathrm{E}-06$

2.17E-06

2. $16 \mathrm{E}-06$

2.03E-06

$1.93 \mathrm{E}-06$

1.91E-06

$1.86 \mathrm{E}-06$

$1.82 \mathrm{E}-06$

$1.81 \mathrm{E}-06$

$1.80 \mathrm{E}-06$

$1.74 \mathrm{E}-06$

$1.70 \mathrm{E}-06$

$1.68 \mathrm{E}-06$

$1.68 \mathrm{E}-06$

1. 66E-06

$1.60 \mathrm{E}-06$

$1.50 \mathrm{E}-06$

1. $40 \mathrm{E}-06$

1. $39 \mathrm{E}-06$

1. 33E-06

1. 31E-06

1. 27E-06

1. 26E-06

1. $25 \mathrm{E}-06$

$1.23 \mathrm{E}-06$

$1.22 \mathrm{E}-06$

$1.14 \mathrm{E}-06$

$1.13 \mathrm{E}-06$

$1.03 \mathrm{E}-06$

$1.03 \mathrm{E}-06$

$1.02 \mathrm{E}-06$

$1.02 \mathrm{E}-06$

$1.00 \mathrm{E}-06$

9.92E-07

$9.72 \mathrm{E}-07$

$9.68 \mathrm{E}-07$

9.37E-07

9. 36E-07

8.99E-07

8.61E-07

8. 53E-07

7.67E-07

7. 37E-07

7. 31E-07

7.26E-07

7. 24E-07

$7.13 \mathrm{E}-07$

7.07E-07

$7.06 \mathrm{E}-07$

$6.80 \mathrm{E}-07$

$6.59 \mathrm{E}-07$

$6.58 \mathrm{E}-07$

$6.49 \mathrm{E}-07$

$6.29 \mathrm{E}-07$

$5.84 \mathrm{E}-07$

$5.60 \mathrm{E}-07$

$5.40 \mathrm{E}-07$

5.11E-07

5.05E-07

4. 70E-07

4. 62E-07

4.62E-07

4. 56E-07

4. 53E-07

4. 38E-07

4. 37E-07
Percent

$4.29 \mathrm{E}-03$
$4.26 \mathrm{E}-03$
$4.24 \mathrm{E}-03$
$4.20 \mathrm{E}-03$
$3.95 \mathrm{E}-03$
$3.75 \mathrm{E}-03$
$3.72 \mathrm{E}-03$
$3.62 \mathrm{E}-03$
$3.55 \mathrm{E}-03$
$3.53 \mathrm{E}-03$
$3.51 \mathrm{E}-03$
$3.40 \mathrm{E}-03$
$3.31 \mathrm{E}-03$
$3.28 \mathrm{E}-03$
$3.28 \mathrm{E}-03$
$3.24 \mathrm{E}-03$
$3.12 \mathrm{E}-03$
$2.93 \mathrm{E}-03$
$2.73 \mathrm{E}-03$
$2.70 \mathrm{E}-03$
$2.58 \mathrm{E}-03$

2. $56 \mathrm{E}-03$

2. 47E-03

2. $45 \mathrm{E}-03$

2. $43 \mathrm{E}-03$

2. $40 \mathrm{E}-03$

2. 38E-03

2. 23E-03

2. 21E-03

2. $00 \mathrm{E}-03$

2. $00 \mathrm{E}-03$

1. 98E-03

1.98E-03

$1.95 \mathrm{E}-03$

1.93E-03

1.89E-03

$1.89 \mathrm{E}-03$

$1.83 \mathrm{E}-03$

$1.82 \mathrm{E}-03$

$1.75 \mathrm{E}-03$

1. $68 \mathrm{E}-03$

1. 66E-03

$1.49 \mathrm{E}-03$

1. 44E-03

1. $42 \mathrm{E}-03$

$1.41 \mathrm{E}-03$

1. $41 \mathrm{E}-03$

1. 39E-03

1. 38E-03

1. 38E-03

1. 33E-03

1. $28 \mathrm{E}-03$

1. $28 \mathrm{E}-03$

1. $26 \mathrm{E}-03$

1. $22 \mathrm{E}-03$

1. 14E-03

1. 09E-03

1. 05E-03

9.95E-04

9. 84E-04

9. $15 \mathrm{E}-04$

9. $00 \mathrm{E}-04$

9.00E-04

8. 88E-04

8. 82E-04

8.53E-04

8. 52E-04 
DOWNWIND DISTANCE $=1.00 \mathrm{E}+03(\mathrm{~m})$

SORTED BY PERCENT OF EFFECTIVE DOSE

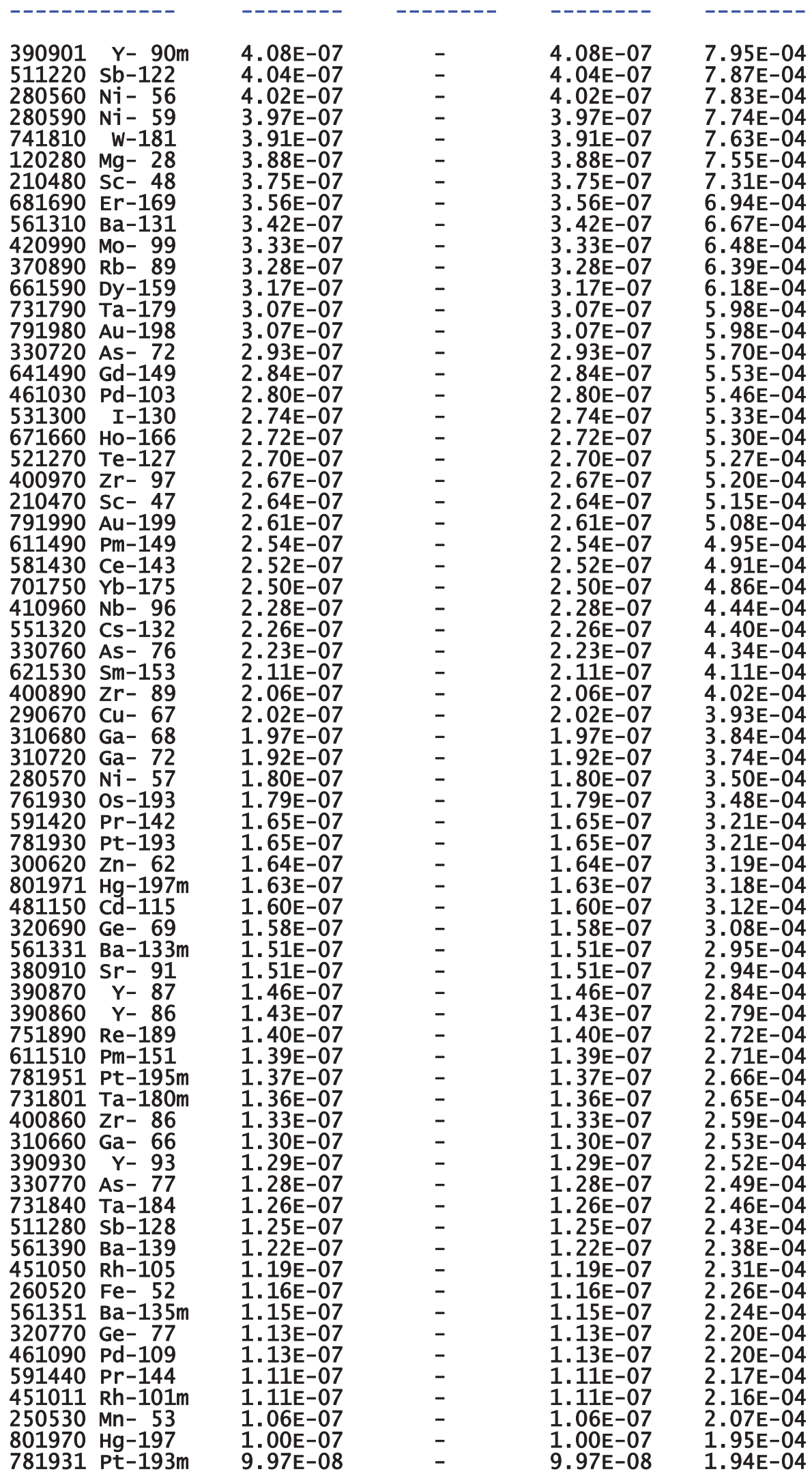


DOWNWIND DISTANCE $=1.00 \mathrm{E}+03(\mathrm{~m})$ SORTED BY PERCENT OF EFFECTIVE DOSE

\section{NUCLIDE} INTERNAL

$$
\begin{array}{r}
9.7 \\
9.4 \\
8.89 \\
8.8 \\
8.3 \\
8.2 \\
8.2 \\
8.2 \\
8.1 \\
7.5 \\
7.4 \\
7.4 \\
6.3 \\
6.8 \\
6.5 \\
6.4 \\
6.4
\end{array}
$$

$781910 \mathrm{Pt}-191$

$340730 \mathrm{Se}-73$

$751822 \mathrm{Re}-182 \mathrm{a}$ 631503 Eu-150b 390920 Y- 92

751870 Re-187

$441050 \mathrm{Ru}-105$

$471120 \mathrm{Ag}-112$

$591450 \mathrm{Pr}-145$

$521290 \mathrm{Te}-129$

420931 Mo- $93 \mathrm{~m}$

230490 V- 49

$551310 \mathrm{Cs}-131$

521331 Te-133m

761911 os $-191 \mathrm{~m}$

571410 La-141

491131 In-113m

$190430 \mathrm{~K}-43$

501100 Sn-110

$300711 \mathrm{Zn}-71 \mathrm{~m}$

$40070 \mathrm{Be}-7$

$440970 \mathrm{Ru}-97$

$350770 \mathrm{Br}-77$

$310730 \mathrm{Ga}-73$

812000 T1-200

190420 K- 42

$240510 \mathrm{Cr}-51$

$290640 \mathrm{Cu}-64$

611500 Pm-150

501270 Sn-127

$711761 \mathrm{Lu}-176 \mathrm{~m}$

$250560 \mathrm{Mn}-56$

491171 In-117m

451061 Rh-106m

571420 La-142

781970 Pt-197

330780 As - 78

$481171 \mathrm{Cd}-117 \mathrm{~m}$

$220450 \mathrm{Ti}-45$

551290 Cs-129

$280650 \mathrm{Ni}-65$

$320780 \mathrm{Ge}-78$

$601490 \mathrm{Nd}-149$

771904 Ir-190n

$350801 \mathrm{Br}-80 \mathrm{~m}$

$140310 \mathrm{Si}-31$

491151 In-115m

501280 Sn-128
EXTERNAL

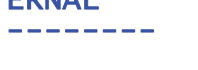

$9.47 \mathrm{E}-08$
$8.89 \mathrm{E}-08$
$8.84 \mathrm{E}-08$

$8.35 \mathrm{E}-08$

$8.26 \mathrm{E}-08$

$8.22 \mathrm{E}-08$

$8.22 \mathrm{E}-08$

$8.17 \mathrm{E}-08$

$7.54 \mathrm{E}-08$

$7.44 \mathrm{E}-08$

$7.43 \mathrm{E}-08$

$7.33 \mathrm{E}-08$

6. $99 \mathrm{E}-08$

$6.81 \mathrm{E}-08$

$6.55 \mathrm{E}-08$

$6.49 \mathrm{E}-08$

$6.45 \mathrm{E}-08$

6. $39 \mathrm{E}-08$

$6.27 \mathrm{E}-08$

$6.12 \mathrm{E}-08$

$6.04 \mathrm{E}-08$

$5.77 \mathrm{E}-08$

5. 54E-08

5. $31 \mathrm{E}-08$

$5.26 \mathrm{E}-08$

$5.17 \mathrm{E}-08$

$5.17 \mathrm{E}-08$

$5.12 \mathrm{E}-08$

$4.95 \mathrm{E}-08$

4. $94 \mathrm{E}-08$

$4.86 \mathrm{E}-08$

4. $80 \mathrm{E}-08$

$4.80 \mathrm{E}-08$

$4.78 \mathrm{E}-08$

$4.68 \mathrm{E}-08$

$4.65 \mathrm{E}-08$

$4.52 \mathrm{E}-08$

4. $51 \mathrm{E}-08$

4. $42 \mathrm{E}-08$

$4.29 \mathrm{E}-08$

$4.25 \mathrm{E}-08$

$4.01 \mathrm{E}-08$

3. $98 \mathrm{E}-08$

$3.75 \mathrm{E}-08$

$3.70 \mathrm{E}-08$

3. $61 \mathrm{E}-08$

$3.53 \mathrm{E}-08$

3. $42 \mathrm{E}-08$

3. $36 \mathrm{E}-08$

3. $24 \mathrm{E}-08$

$3.12 \mathrm{E}-08$

2. $91 \mathrm{E}-08$

2. $88 \mathrm{E}-08$

$2.77 \mathrm{E}-08$

2. $66 \mathrm{E}-08$

2. $61 \mathrm{E}-08$

2. $56 \mathrm{E}-08$

2. $53 \mathrm{E}-08$

2. $42 \mathrm{E}-08$

2. $35 \mathrm{E}-08$

2. $32 \mathrm{E}-08$

2. $30 \mathrm{E}-08$

$2.17 \mathrm{E}-08$

2. $14 \mathrm{E}-08$

$2.08 \mathrm{E}-08$

2. $05 \mathrm{E}-08$
TOTAL

9. $73 \mathrm{E}-08$

$9.47 \mathrm{E}-08$

8. $89 \mathrm{E}-08$

8. 84E-08

$8.35 \mathrm{E}-08$

8. $26 \mathrm{E}-08$

$8.22 \mathrm{E}-08$

8.22E-08

8.17E-08

7. $54 \mathrm{E}-08$

7. $44 \mathrm{E}-08$

7. $43 \mathrm{E}-08$

7. 33E-08

$6.99 \mathrm{E}-08$

$6.81 \mathrm{E}-08$

$6.55 \mathrm{E}-08$

$6.49 \mathrm{E}-08$

$6.45 \mathrm{E}-08$

6. $39 \mathrm{E}-08$

$6.27 \mathrm{E}-08$

$6.12 \mathrm{E}-08$

6.04E-08

$5.77 \mathrm{E}-08$

5. 54E-08

5. $31 \mathrm{E}-08$

5. $26 \mathrm{E}-08$

$5.17 \mathrm{E}-08$

$5.17 \mathrm{E}-08$

$5.12 \mathrm{E}-08$

$4.95 \mathrm{E}-08$

4. $94 \mathrm{E}-08$

$4.86 \mathrm{E}-08$

$4.80 \mathrm{E}-08$

$4.80 \mathrm{E}-08$

$4.78 \mathrm{E}-08$

$4.68 \mathrm{E}-08$

$4.65 \mathrm{E}-08$

$4.52 \mathrm{E}-08$

4. $51 \mathrm{E}-08$

$4.42 \mathrm{E}-08$

4. $29 \mathrm{E}-08$

$4.25 \mathrm{E}-08$

$4.01 \mathrm{E}-08$

3. $98 \mathrm{E}-08$

$3.75 \mathrm{E}-08$

$3.70 \mathrm{E}-08$

3. $61 \mathrm{E}-08$

3. $53 \mathrm{E}-08$

3. $42 \mathrm{E}-08$

3. $36 \mathrm{E}-08$

3. $24 \mathrm{E}-08$

3. $12 \mathrm{E}-08$

2.91E-08

$2.88 \mathrm{E}-08$

$2.77 \mathrm{E}-08$

2. $66 \mathrm{E}-08$

2. $61 \mathrm{E}-08$

2. $56 \mathrm{E}-08$

2. $53 \mathrm{E}-08$

2. $42 \mathrm{E}-08$

2. $35 \mathrm{E}-08$

2. $32 \mathrm{E}-08$

2. $30 \mathrm{E}-08$

$2.17 \mathrm{E}-08$

2. $14 \mathrm{E}-08$

$2.08 \mathrm{E}-08$

2. $05 \mathrm{E}-08$
Percent

1. $90 \mathrm{E}-04$

1.84E-04

$1.72 \mathrm{E}-04$

$1.63 \mathrm{E}-04$

$1.61 \mathrm{E}-04$

1. $60 \mathrm{E}-04$

1. $60 \mathrm{E}-04$

1. $59 \mathrm{E}-04$

$1.47 \mathrm{E}-04$

$1.45 \mathrm{E}-04$

$1.45 \mathrm{E}-04$

1. $43 \mathrm{E}-04$

$1.36 \mathrm{E}-04$

1. 33E-04

1. $28 \mathrm{E}-04$

$1.26 \mathrm{E}-04$

$1.26 \mathrm{E}-04$

$1.25 \mathrm{E}-04$

$1.22 \mathrm{E}-04$

$1.19 \mathrm{E}-04$

$1.18 \mathrm{E}-04$

$1.12 \mathrm{E}-04$

$1.08 \mathrm{E}-04$

1. $03 \mathrm{E}-04$

$1.02 \mathrm{E}-04$

1. $01 \mathrm{E}-04$

1. $01 \mathrm{E}-04$

9.97E-05

9.65E-05

9. $62 \mathrm{E}-05$

9. $46 \mathrm{E}-05$

9. $35 \mathrm{E}-05$

9. $35 \mathrm{E}-05$

9. 31E-05

9.11E-05

9.07E-05

8. 81E-05

$8.78 \mathrm{E}-05$

8. $61 \mathrm{E}-05$

8. $36 \mathrm{E}-05$

8. 28E-05

7. $81 \mathrm{E}-05$

7.76E-05

7. 31E-05

$7.22 \mathrm{E}-05$

7.03E-05

6.87E-05

6. 66E-05

6. $54 \mathrm{E}-05$

6. 32E-05

$6.08 \mathrm{E}-05$

5. 67E-05

5. 61E-05

5. $39 \mathrm{E}-05$

$5.18 \mathrm{E}-05$

5. $09 \mathrm{E}-05$

4. $99 \mathrm{E}-05$

$4.94 \mathrm{E}-05$

4.72E-05

4. 57E-05

4. 52E-05

$4.48 \mathrm{E}-05$

4. $23 \mathrm{E}-05$

$4.17 \mathrm{E}-05$

4. $04 \mathrm{E}-05$

3. $99 \mathrm{E}-05$ 
DOWNWIND DISTANCE $=1.00 \mathrm{E}+03(\mathrm{~m})$

SORTED BY PERCENT OF EFFECTIVE DOSE

NUCLIDE

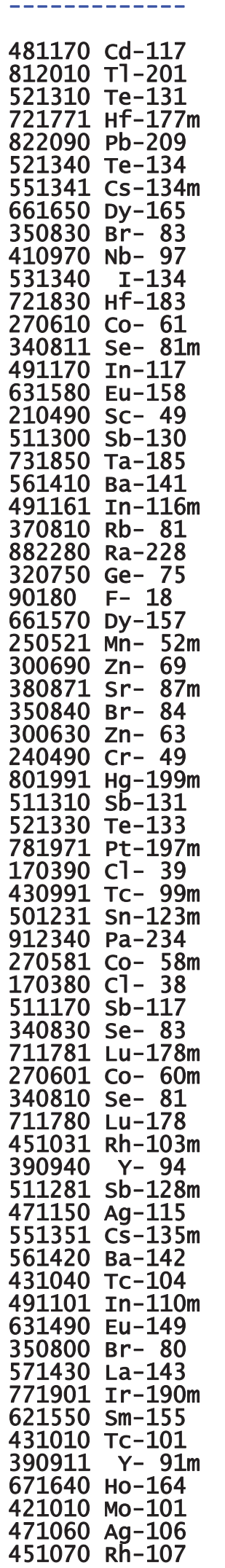
INTERNAL

$$
\begin{aligned}
& 2.03 \\
& 1.9 \\
& 1.9 \\
& 1.9 \\
& 1.8 \\
& 1.6 \\
& 1.6 \\
& 1.6 \\
& 1.4 \\
& 1.3 \\
& 1.3 \\
& 1.3 \\
& 1.2 \\
& 1.1 \\
& 1.1 \\
& 1.0 \\
& 1.0 \\
& 1.01 \\
& 1.0 \\
& 9.9 \\
& 9.7 \\
& 9.6 \\
& 9.6 \\
& 9.1 \\
& 8
\end{aligned}
$$$$
\begin{aligned}
& 2.03 \mathrm{E}-0 \\
& 1.98 \mathrm{E}-0 \\
& 1.91 \mathrm{E}-08 \\
& 1.91 \mathrm{E}-0 \\
& 1.85 \mathrm{E}-0 \\
& 1.65 \mathrm{E}-0
\end{aligned}
$$

1. $64 \mathrm{E}-08$

1. $60 \mathrm{E}-08$

$1.42 \mathrm{E}-08$

1.38E-08

1. $36 \mathrm{E}-08$

1. 30E-08

1. $29 \mathrm{E}-08$

$1.12 \mathrm{E}-08$

1.10E-08

$1.08 \mathrm{E}-08$

$1.01 \mathrm{E}-08$

$1.01 \mathrm{E}-08$

$1.00 \mathrm{E}-08$

9.94E-09

9.74E-09

$9.68 \mathrm{E}-09$

$9.64 \mathrm{E}-09$

$10 E-09$

8. $92 \mathrm{E}-09$

$8.79 \mathrm{E}-09$

8.72E-09

8. 50E-09

7. $90 \mathrm{E}-09$

6. 99E-09

6.97E-09

$6.88 \mathrm{E}-09$

6. 31E-09

6. 26E-09

$6.24 \mathrm{E}-09$

6.02E-09

5. $90 \mathrm{E}-09$

5. 79E-09

5. 20E-09

5. 04E-09

5. 04E-09

4. 90E-09

4. 64E-09

4. 54E-09

4. 51E-09

4. 26E-09

4.15E-09

4.13E-09

4. 02E-09

4. $02 \mathrm{E}-09$

3. 89E-09

3. $56 \mathrm{E}-09$

3. 44E-09

3. $43 \mathrm{E}-09$

3. 42E-09

3. 23E-09

3. $10 \mathrm{E}-09$

2.79E-09

2. 40E-09

2. 39E-09

2. 35E-09

2. 33E-09

2. 31E-09

$2.28 \mathrm{E}-09$

2. $26 \mathrm{E}-09$

2. $25 \mathrm{E}-09$

2. 25E-09
EXTERNAL
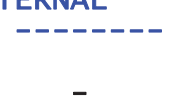

-
-
-

$-$

$-$

$-$

$-$

-

-

$-$

$-$

$-$

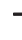

-

$-$

-

$-$

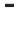

TOTAL

Percent

2. $03 \mathrm{E}-08$

$1.98 \mathrm{E}-08$

1. $91 \mathrm{E}-08$

$1.91 \mathrm{E}-08$

$1.85 \mathrm{E}-08$

$1.65 \mathrm{E}-08$

1. $64 \mathrm{E}-08$

1. $60 \mathrm{E}-08$

$1.42 \mathrm{E}-08$

$1.38 \mathrm{E}-08$

1. $36 \mathrm{E}-08$

1. $30 \mathrm{E}-08$

$1.29 \mathrm{E}-08$

$1.12 \mathrm{E}-08$

$1.10 \mathrm{E}-08$

$1.08 \mathrm{E}-08$

$1.01 \mathrm{E}-08$

$1.01 \mathrm{E}-08$

1. $00 \mathrm{E}-08$

9.94E-09

9.74E-09

9.68E-09

9.64E-09

9. $10 \mathrm{E}-09$

8. $92 \mathrm{E}-09$

8.79E-09

8.72E-09

8. $50 \mathrm{E}-09$

7. $90 \mathrm{E}-09$

$6.99 \mathrm{E}-09$

6.97E-09

6. 88E-09

6.31E-09

$6.26 \mathrm{E}-09$

6.24E-09

$6.02 \mathrm{E}-09$

$5.90 \mathrm{E}-09$

5.79E-09

5. $20 \mathrm{E}-09$

5.04E-09

5.04E-09

4. $90 \mathrm{E}-09$

4. 64E-09

4. 54E-09

4. $51 \mathrm{E}-09$

4. $26 \mathrm{E}-09$

4.15E-09

$4.13 \mathrm{E}-09$

4.02E-09

$4.02 \mathrm{E}-09$

3. $89 \mathrm{E}-09$

3. $56 \mathrm{E}-09$

3. $44 \mathrm{E}-09$

3. $43 \mathrm{E}-09$

3. $42 \mathrm{E}-09$

3. $23 \mathrm{E}-09$

3.10E-09

2. $79 \mathrm{E}-09$

2. $40 \mathrm{E}-09$

2. 39E-09

2. $35 \mathrm{E}-09$

2.33E-09

2. 31E-09

2. $28 \mathrm{E}-09$

2. $26 \mathrm{E}-09$

2. $25 \mathrm{E}-09$

2. $25 \mathrm{E}-09$
3. $95 \mathrm{E}-05$

3. $85 \mathrm{E}-05$

$3.73 \mathrm{E}-05$

3.72E-05

3. $60 \mathrm{E}-05$

3. $22 \mathrm{E}-05$

$3.20 \mathrm{E}-05$

3. $12 \mathrm{E}-05$

$2.76 \mathrm{E}-05$

2. 69E-05

2. $65 \mathrm{E}-05$

2. $53 \mathrm{E}-05$

2. $51 \mathrm{E}-05$

2.19E-05

2.14E-05

2. $10 \mathrm{E}-05$

$1.97 \mathrm{E}-05$

1. $97 \mathrm{E}-05$

1. $96 \mathrm{E}-05$

1. $94 \mathrm{E}-05$

1. $90 \mathrm{E}-05$

1. 89E-05

1. $88 \mathrm{E}-05$

$1.77 \mathrm{E}-05$

$1.74 \mathrm{E}-05$

$1.71 \mathrm{E}-05$

1.70E-05

$1.66 \mathrm{E}-05$

1. $54 \mathrm{E}-05$

1. $36 \mathrm{E}-05$

1. $36 \mathrm{E}-05$

1. $34 \mathrm{E}-05$

1. $23 \mathrm{E}-05$

$1.22 \mathrm{E}-05$

$1.22 \mathrm{E}-05$

$1.17 \mathrm{E}-05$

1. $15 \mathrm{E}-05$

$1.13 \mathrm{E}-05$

$1.01 \mathrm{E}-05$

9. 82E-06

$9.81 \mathrm{E}-06$

9. 54E-06

9.03E-06

8. 84E-06

8.79E-06

8. $29 \mathrm{E}-06$

8.08E-06

8.04E-06

7. 84E-06

$7.82 \mathrm{E}-06$

7. $57 \mathrm{E}-06$

6. $93 \mathrm{E}-06$

6. $69 \mathrm{E}-06$

6. $68 \mathrm{E}-06$

$6.66 \mathrm{E}-06$

$6.29 \mathrm{E}-06$

$6.05 \mathrm{E}-06$

5. $43 \mathrm{E}-06$

$4.67 \mathrm{E}-06$

4. $65 \mathrm{E}-06$

$4.58 \mathrm{E}-06$

4. 53E-06

4. $50 \mathrm{E}-06$

4. $44 \mathrm{E}-06$

4. $40 \mathrm{E}-06$

4. $39 \mathrm{E}-06$

4. $39 \mathrm{E}-06$ 
DOWNWIND DISTANCE $=1.00 \mathrm{E}+03(\mathrm{~m})$

SORTED BY PERCENT OF EFFECTIVE DOSE

NUCLIDE INTERNAL EXTERNAL

-------------

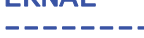

TOTAL

2.23E-09 -

491191 In-119m

$310700 \mathrm{Ga}-70$

$731821 \mathrm{Ta}-182 \mathrm{~m}$

$390950 \quad \mathrm{Y}-95$

$531280 \quad \mathrm{I}-128$

270621 Co- $62 \mathrm{~m}$

430961 TC- $96 \mathrm{~m}$

761891 os $-189 \mathrm{~m}$

$591470 \mathrm{Pr}-147$

$751881 \mathrm{Re}-188 \mathrm{~m}$

$601410 \mathrm{Nd}-141$

$601510 \mathrm{Nd}-151$

$380851 \mathrm{Sr}-85 \mathrm{~m}$

$731860 \mathrm{Ta}-186$

741790 w-179

902310 Th-231

892280 AC-228

922340 U-234

$912310 \mathrm{~Pa}-231$

$882260 \mathrm{Ra}-226$

902300 Th-230

TOTALS

WARNINGS

\section{$-$}

$-$

$-$

$2.03 \mathrm{E}-09$

$1.99 \mathrm{E}-09$

1. $91 \mathrm{E}-09$

$1.89 \mathrm{E}-09$

$1.69 \mathrm{E}-09$

1. $59 \mathrm{E}-09$

$1.55 \mathrm{E}-09$

$1.49 \mathrm{E}-09$

$1.46 \mathrm{E}-09$

1. $34 \mathrm{E}-09$

1. $20 \mathrm{E}-09$

9. $96 \mathrm{E}-10$

9.42E-10

1. $70 \mathrm{E}-10$

5. $22 \mathrm{E}-12$

$4.97 \mathrm{E}-12$

$8.00 \mathrm{E}-13$

7. 31E-14

1.79E-17

2.04E-18

5. 13E-02
2. $23 \mathrm{E}-09$

2. 18E-09

2. $03 \mathrm{E}-09$

1. 99E-09

1.91E-09

1. 89E-09

1.69E-09

1. 59E-09

1. 55E-09

1. $49 \mathrm{E}-09$

1. 46E-09

1. 34E-09

1. $20 \mathrm{E}-09$

9. $96 \mathrm{E}-10$

9. $42 \mathrm{E}-10$

1. $70 \mathrm{E}-10$

5. 22E-12

4. $97 \mathrm{E}-12$

8. 00E-13

7. 31E-14

1. $79 \mathrm{E}-17$

2. 04E-18

5. 13E-02
Percent

4. 35E-06

4. $26 \mathrm{E}-06$

3. $96 \mathrm{E}-06$

3. $88 \mathrm{E}-06$

$3.71 \mathrm{E}-06$

3. $68 \mathrm{E}-06$

3. $30 \mathrm{E}-06$

3. $11 \mathrm{E}-06$

3. $02 \mathrm{E}-06$

2.91E-06

2. 85E-06

2. $62 \mathrm{E}-06$

2. $35 \mathrm{E}-06$

$1.94 \mathrm{E}-06$

1. 83E-06

3. $31 \mathrm{E}-07$

$1.02 \mathrm{E}-08$

9.68E-09

1. $56 \mathrm{E}-09$

1. $42 \mathrm{E}-10$

3. $48 \mathrm{E}-14$

3. $98 \mathrm{E}-15$

1. $00 \mathrm{E}+02$

NO AIR IMMERSION OR CLOUD GAMMA DOSE CALCULATIONS WERE MADE EXECUTION TIME

1. 50E+00 SECONDS 